\section{Overview and Background}

This is a course for computer system designers and builders, and for people who want to really understand how systems work, especially concurrent, distributed, and fault-tolerant systems.

\section{The course teaches you}

how to write precise specifications for any kind of computer system,

what it means for an implementation to satisfy a specification, and

how to prove that it does.

It also shows you how to use the same methods less formally, and gives you some suggestions for deciding how much formality is appropriate (less formality means less work, and often a more understandable spec, but also more chance to overlook an important detail).

The course also teaches you a lot about the topics in computer systems that we think are the most important: persistent storage, concurrency, naming, networks, distributed systems, transactions, fault tolerance, and caching. The emphasis is on

careful specifications of subtle and sometimes complicated things,

the important ideas behind good implementations, and

how to understand what makes them actually work

We spend most of our time on specific topics, but we use the general techniques throughout. We emphasize the ideas that different kinds of computer system have in common, even when they have different names.

The course uses a formal language called Spec for writing specs and implementations; you can think of it as a very high level programming language. There is a good deal of written introductory material on Spec (explanations and finger exercises) as well as a reference manual and a formal semantics. We introduce Spec ideas in class as we use them, but we do not devote class time to teaching Spec per se; we expect you to learn it on your own from the handouts.

Because we write specs and do proofs, you need to know something about logic. Since many people don't, there is a concise treatment of the logic you will need at the end of this handout

This is not a course in computer architecture, networks, operating systems, or databases. We will not talk in detail about how to implement pipelines, memory interconnects, multiprocessors, routers, data link protocols, network management, virtual memory, scheduling, resource allocation, SQL, relational integrity, or TP monitors, although we will deal with many of the ideas that underlie these mechanisms.

\section{Topics}

General

Specifications as state machines.

The Spec language for describing state machines (writing specs and implementations).

What it means to implement a spec.

Using abstraction functions and invariants to prove that a program implements a spec.
What it means to have a crash.

What every system builder needs to know about performance.

Specific

Disks and file systems.

Practical concurrency using mutexes (locks) and condition variables; deadlock

Hard concurrency (without locking): models, specs, proofs, and examples.

Transactions: simple, cached, concurrent, distributed.

Naming: principles, specs, and examples.

Distributed systems: communication, fault-tolerance, and autonomy.

Networking: links, switches, reliable messages and connections.

Remote procedure call and network objects.

Fault-tolerance, availability, consensus and replication.

Caching and distributed shared memory.

Previous editions of the course have also covered security (authentication, authorization, encryption, trust) and system management, but this year we are omitting these topics in order to spend more time on concurrency and semantics and to leave room for project presentations.

\section{Prerequisites}

There are no formal prerequisites for the course. However, we assume some knowledge both of computer systems and of mathematics. If you have taken 6.033 and 6.042, you should be in good shape. If you are missing some of this knowledge you can pick it up as we go, but if you are missing a lot of it you can expect to have serious trouble. It's also important to have a certain amount of maturity: enough experience with systems and mathematics to feel comfortable with the basic notions and to have some reliable intuition.

If you know the meaning of the following words, you have the necessary background. If a lot of them are unfamiliar, this course is probably not for you.

Systems

Cache, virtual memory, page table, pipeline

Process, scheduler, address space, priority

Thread, mutual exclusion (locking), semaphore, producer-consumer, deadlock

Transaction, commit, availability, relational data base, query, join

File system, directory, path name, striping, RAID

LAN, switch, routing, connection, flow control, congestion

Capability, access control list, principal (subject)

If you have not already studied Lampson's paper on hints for system design, you should do so as background for this course. It is Butler Lampson, Hints for computer system design, Proceedings of the Ninth ACM Symposium on Operating Systems Principles, October 1983, pp 33-48. There is a pointer to it on the course Web page. 


\section{Programming}

Invariant, precondition, weakest precondition, fixed point

Procedure, recursion, stack

Data type, sub-type, type-checking, abstraction, representation

Object, method, inheritance

Data structures: list, hash table, binary search, B-tree, graph

\section{Mathematics}

Function, relation, set, transitive closure

Logic: proof, induction, de Morgan's laws, implication, predicate, quantifier

Probability: independent events, sampling, Poisson distribution

State machine, context-free grammar

Computational complexity, unsolvable problem

If you haven't been exposed to formal logic, you should study the summary at the end of this handout.

\section{References}

These are places to look when you want more information about some topic covered or alluded to in the course, or when you want to follow current research. You might also wish to consult Prof. Saltzer's bibliography for 6.033, which you can find on the course web page.

\section{Books}

Some of these are fat books better suited for reference than for reading cover to cover, especially Cormen, Leiserson, and Rivest, Jain, Mullender, Hennessy and Patterson, and Gray and Reuter. But the last two are pretty easy to read in spite of their encyclopedic character.

Systems programming: Greg Nelson, ed., Systems Programming with Modula-3, Prentice-Hall, 1991. Describes the language, which has all the useful features of $\mathrm{C}++$ but is much simpler and less error-prone, and also shows how to use it for concurrency (a version of chapter 4 is a handout in this course), an efficiently customizable I/O streams package, and a window system.

Performance: Jon Bentley, Writing Efficient Programs, Prentice-Hall, 1982. Short, concrete, and practical. Raj Jain, The Art of Computer Systems Performance Analysis, Wiley, 1991. Tells you much more than you need to know about this subject, but does have a lot of realistic examples.

Algorithms and data structures: Robert Sedgwick, Algorithms, Addison-Wesley, 1983. Short, and usually tells you what you need to know. Tom Cormen, Charles Leiserson, and Ron Rivest, Introduction to Algorithms, McGraw-Hill, 1989. Comprehensive, and sometimes valuable for that reason, but usually tells you a lot more than you need to know.

Distributed algorithms: Nancy Lynch, Distributed Algorithms, Morgan Kaufmann, 1996. The bible for distributed algorithms. Comprehensive, but a much more formal treatment than in this course. The topic is algorithms, not systems.
Computer architecture: John Hennessy and David Patterson, Computer Architecture: A Quantitative Approach, 2nd edition, Morgan Kaufmann, 1995. The bible for computer architecture. The second edition has lots of interesting new material, especially on multiprocessor memory systems and interconnection networks. There's also a good appendix on computer arithmetic; it's useful to know where to find this information, though it has nothing to do with this course.

Transactions, data bases, and fault-tolerance: Jim Gray and Andreas Reuter, Transaction Processing: Concepts and Techniques, Morgan Kaufmann, 1993. The bible for transaction processing, with much good material on data bases as well; it includes a lot of practical information that doesn't appear elsewhere in the literature.

Networks: Radia Perlman, Interconnections: Bridges and Routers, Addison-Wesley, 1992. Not exactly the bible for networking, but tells you nearly everything you might want to know about how packets are actually switched in computer networks.

Distributed systems: Sape Mullender, ed., Distributed Systems, 2nd ed., Addison-Wesley, 1993 A compendium by many authors that covers the field fairly well. Some chapters are much more theoretical than this course. Chapters 10 and 11 are handouts in this course. Chapters 1, 2, 8, and 12 are also recommended. Chapters 16 and 17 are the best you can do to learn about real-time computing; unfortunately, that is not saying much.

User interfaces: Alan Cooper, About Face, IDG Books, 1995. Principles, lots of examples, and opinionated advice, much of it good, from the original designer of Visual Basic.

Journals

You can find all of these in the LCS reading room. The cryptic strings in brackets are call numbers there. You can also find the last few years of the ACM publications in the ACM digital library at www.acm.org.

For the current literature, the best sources are the proceedings of the following conferences. 'Sig' is short for "Special Interest Group", a subdivision of the ACM that deals with one field of computing. The relevant ones for systems are SigArch for computer architecture, SigPlan for programming languages, SigOps for operating systems, SigComm for communications, SigMod for data bases, and SigMetrics for performance measurement and analysis.

Symposium on Operating Systems Principles (SOSP; published as special issues of ACM SigOps Operating Systems Review; fall of odd-numbered years) [P4.35.06]

Operating Systems Design and Implementation (OSDI; Usenix Association, now published as special issues of ACM SigOps Review; fall of even-numbered years, except spring 1999 instead of fall 1998) [P4.35.U71]

Architectural Support for Programming Languages and Operating Systems (ASPLOS published as special issues of ACM SigOps Operating Systems Review, SigArch Computer Architecture News, or SigPlan Notices; fall of even-numbered years) [P6.29.A7] 
Applications, Technologies, Architecture, and Protocols for Computer Communication, (SigComm conference; published as special issues of ACM SigComm Computer

Communication Review; annual) [P6.24.D31]

Principles of Distributed Computing (PODC; ACM; annual) [P4.32.D57]

Very Large Data Bases (VLDB; Morgan Kaufmann; annual) [P4.33.V4]

International Symposium on Computer Architecture (ISCA; published as special issues of ACM SigArch Computer Architecture News; annual) [P6.20.C6]

Less up to date, but more selective, are the journals. Often papers in these journals are revised versions of papers from the conferences listed above.

ACM Transactions on Computer Systems

ACM Transactions on Database Systems

ACM Transactions on Programming Languages and Systems

There are often good survey articles in the less technical IEEE journals:

IEEE Computer, Networks, Communication, Software

The Internet Requests for Comments (RFC's) can be reached from

http://ds.internic.net/ds/rfc-index.html

\section{Rudiments of logic}

Propositional logic

The basic type is Bool, which contains two elements true and false. Expressions in these operators (and the other ones introduced later) are called 'propositions'.

Basic operators. These are $\wedge$ (and), $\vee$ (or), and $\sim$ (not). ${ }^{1}$ The meaning of these operators can be conveniently given by a 'truth table' which lists the value of a op b for each possible combination of values of $a$ and $b$ (the operators on the right are discussed later) along with some popular names for certain expressions and their operands.

\begin{tabular}{|cc|cccccc|}
\hline & & negation & conjunction & disjunction & equality & & implication \\
\hline & & not & and & or & & & implies \\
$\mathrm{a}$ & $\mathrm{b}$ & $\sim \mathrm{a}$ & $\mathrm{a} \wedge \mathrm{b}$ & $\mathrm{a} \vee \mathrm{b}$ & $\mathrm{a}=\mathrm{b}$ & $\mathrm{a} \neq \mathrm{b}$ & $\mathrm{a} \Rightarrow \mathrm{b}$ \\
\hline $\mathrm{T}$ & $\mathrm{T}$ & $\mathrm{F}$ & $\mathrm{T}$ & $\mathrm{T}$ & $\mathrm{T}$ & $\mathrm{F}$ & $\mathrm{T}$ \\
$\mathrm{T}$ & $\mathrm{F}$ & & $\mathrm{F}$ & $\mathrm{T}$ & $\mathrm{F}$ & $\mathrm{T}$ & $\mathrm{F}$ \\
$\mathrm{F}$ & $\mathrm{T}$ & $\mathrm{T}$ & $\mathrm{F}$ & $\mathrm{T}$ & $\mathrm{F}$ & $\mathrm{T}$ & $\mathrm{T}$ \\
$\mathrm{F}$ & $\mathrm{F}$ & & $\mathrm{F}$ & $\mathrm{F}$ & $\mathrm{T}$ & $\mathrm{F}$ & $\mathrm{T}$ \\
\hline \multicolumn{2}{|l}{ name of $\mathrm{a}$} & & conjunct & disjunct & & & antecedent \\
\multicolumn{2}{l}{ name of $\mathrm{b}$} & & conjunct & disjunct & & & consequent \\
\hline
\end{tabular}

Note: In Spec we write $==>$ instead of the $\Rightarrow$ that mathematicians use for implication. Logician write $\supset$ for implication, which looks different but is shaped like the $>$ part of $\Rightarrow$.

In case you have an expression that you can't simplify, you can always work out its truth value by exhaustively enumerating the cases in truth table style. Since the table has only four rows, there are only 16 Boolean operators, one for each possible arrangement of $\mathrm{T}$ and $\mathrm{F}$ in a column. Most of the ones not listed don't have common names, though 'not and' is called 'nand' and 'not or' is called 'nor' by logic designers.

The $\wedge$ and $\vee$ operators are

commutative and

associative and

distribute over each other.

That is, they are just like * (times) and + (plus) on integers, except that + doesn't distribute over *: $a+(b * c) \neq(a+b) *(a+c)$

but $\vee$ does distribute over $\wedge$ :

$a \vee(b \wedge c)=(a \vee b) \wedge(a \vee c)$

An operator that distributes over $\wedge$ is called 'conjunctive'; one that distributes over $\vee$ is called

'disjunctive'. So both $\wedge$ and $\vee$ are both conjunctive and disjunctive. This takes some getting used

${ }^{1}$ It's possible to write all three in terms of the single operator 'nor' or 'nand', but our goal is clarity, not minimality. 
The relation between these operators and $\sim$ is given by DeMorgan's laws (sometimes called the "bubble rule" by logic designers), which say that you can push $\sim$ inside $\wedge$ or $\vee$ by flipping from one to the other:

$$
\begin{aligned}
& \sim(a \wedge b)=\sim a \vee \sim b \\
& \sim(a \vee b)=\sim a \wedge \sim b
\end{aligned}
$$

Because Bool is the result type of relations like =, we can write expressions that mix up relations with other operators in ways that are impossible for any other type. Notably

$$
(\mathrm{a}=\mathrm{b})=((\mathrm{a} \wedge \mathrm{b}) \vee(\sim \mathrm{a} \wedge \sim \mathrm{b}))
$$

Some people feel that the outer $=$ in this expression is somehow different from the inner one, and write it $\equiv$. Experience suggests, however, that this is often a harmful distinction to make.

Implication. We can define an ordering on Bool with false > true, that is, false is greater than true. The non-strict version of this ordering is called 'implication' and written $\Rightarrow$ (rather than $\geq$ or $>=$ as we do with other types; logicians write it $\supset$, which also looks like an ordering symbol). So (true $\Rightarrow$ false) = false (read this as: "true is greater than or equal to false" is false) but all other combinations are true. The expression $a \Rightarrow b$ is pronounced " $a$ implies $b$ ", or "if a then b".

There are lots of rules for manipulating expressions containing $\Rightarrow$; the most useful ones are given below. If you remember that $\Rightarrow$ is an ordering you'll find it easy to remember most of the rules, but if you forget the rules or get confused, you can turn the $\Rightarrow$ into $\vee$ by the rule

$$
(a \Rightarrow b)=\sim a \vee b
$$

and then just use the simpler rules for $\wedge, \vee$, and $\sim$. So remember this even if you forget everything else.

The point of implication is that it tells you when one proposition is stronger than another, in the sense that if the first one is true, the second is also true (because if both $a$ and $a \Rightarrow b$ are true, then $b$ must be true since it can't be false). ${ }^{3}$ So we use implication all the time when reasoning from premises to conclusions. Two more ways to pronounce $a \Rightarrow b$ are " $a$ is stronger than b" and "b follows from a". The second pronunciation suggests that it's sometimes useful to write the operands in the other order, as $\mathrm{b} \Leftarrow \mathrm{a}$, which can also be pronounced " $\mathrm{b}$ is weaker than a" or "b only if a"; this should be no surprise, since we do it with other orderings.

Of course, implication has the properties we expect of an ordering:

Transitive: If $\mathrm{a} \Rightarrow \mathrm{b}$ and $\mathrm{b} \Rightarrow \mathrm{c}$ then $\mathrm{a} \Rightarrow \mathrm{c}{ }^{4}$

${ }^{2}$ It sometimes seems odd that false implies b regardless of what b is, but the "if ... then" form makes it clearer "It sometimes seems odd that false implies $b$ regardless of what $b$ is, but the "if ... then" form makes it cleare
what is going on: if false is true you can conclude anything, but of course it isn't. A proposition that implies what is going on: if false is true you can conclude anything, but of course it isn't. A proposition that imp
false is called 'inconsistent' because it implies anything. Obviously it's bad to think that an inconsistent proposition is true. The most likely way to get into this hole is to think that each of a collection of innocent looking propositions is true when their conjunction turns out to be inconsistent.

${ }_{3}^{3}$ It may also seem odd that false > true rather than the other way around, since true seems better and so should be bigger. But in fact if we want to conclude lots of things, being close to false is better because if fo is true we can conclude anything, but knowing that $t$ rue is true doesn't help at all. Strong propositions are as close to $f a l s e$ as possible; this is logical brinkmanship. For example, a $\wedge$ b is closer to $f a l s e$ than a (there as more values of the variables a and $b$ that make it $f a l s e$ ), and clearly we can conclude more things from it than from

We can also write this $((a \Rightarrow b) \wedge(b \Rightarrow c)) \Rightarrow(a \Rightarrow c)$.
Reflexive: $\mathrm{a} \Rightarrow \mathrm{a}$.

Anti-symmetric: If $\mathrm{a} \Rightarrow \mathrm{b}$ and $\mathrm{b} \Rightarrow \mathrm{a}$ then $\mathrm{a}=\mathrm{b} . \mathrm{s}^{5}$

Furthermore, $\sim$ reverses the sense of implication (this is called the 'contrapositive'):

$(\mathrm{a} \Rightarrow \mathrm{b})=(\sim \mathrm{b} \Rightarrow \sim \mathrm{a})$

More generally, you can move a disjunct on the right to a conjunct on the left by negating it. Thus

$(\mathrm{a} \Rightarrow \mathrm{b} \vee \mathrm{c})=(\mathrm{a} \wedge \sim \mathrm{b} \Rightarrow \mathrm{c})$

As special cases in addition to the contrapositive we have

$(a \Rightarrow b)=(a \wedge \sim b \Rightarrow$ false $)=\sim(a \wedge \sim b) \vee$ false $=\sim a \vee b$

$(\mathrm{a} \Rightarrow \mathrm{b})=$ (true $\Rightarrow \sim \mathrm{a} \vee \mathrm{b}) \quad$ false $\vee \sim \mathrm{a} \vee \mathrm{b}=\sim \mathrm{a} \vee \mathrm{b}$

since false and true are the identities for $\vee$ and $\wedge$.

We say that an operator op is 'monotonic' in an operand if replacing that operand with a stronger (or weaker) one makes the result stronger (or weaker). Precisely, "op is monotonic in its first operand" means that if $a \Rightarrow b$ then ( $a$ op $c$ ) $\Rightarrow$ (b op $c$ ). Both $\wedge$ and $\vee$ are monotonic; in fact, any conjunctive operator is monotonic, because if $a \Rightarrow b$ then $a=(a \wedge b)$, so a op $c=$ $(\mathrm{a} \wedge \mathrm{b})$ op $\mathrm{c}=\mathrm{a}$ op $\mathrm{c} \wedge \mathrm{b}$ op $\mathrm{c} \Rightarrow \mathrm{b}$ op $\mathrm{c}$.

If you know what a lattice is, you will find it useful to know that the set of propositions forms a lattice with $\Rightarrow$ as its ordering and (remember, think of $\Rightarrow$ as "greater than or equal"):

top $\quad$ false

bottom $=$ true

meet $=\wedge \quad$ least upper bound, $\quad$ so $(a \wedge b) \Rightarrow a$ and $(a \wedge b) \Rightarrow b$ join $\quad=\vee \quad$ greatest lower bound, so $a \Rightarrow(a \vee b)$ and $b \Rightarrow(a \vee b)$

This suggests two more expressions that are equivalent to $a \Rightarrow b$ :

$$
(a \Rightarrow b)=(a=(a \wedge b)) \quad \begin{aligned}
& \text { 'and'ing a weaker term makes no difference, } \\
& \text { because } a \Rightarrow b \text { iff } a=\text { least upper bound }(a, b) .
\end{aligned}
$$

$(a \Rightarrow b)=(b=(a \vee b)) \quad$ 'or'ing a stronger term makes no difference, because $\mathrm{a} \Rightarrow \mathrm{b}$ iff $\mathrm{b}=$ greatest lower bound $(\mathrm{a}, \mathrm{b})$.

Predicate logic

Propositions that have free variables, like $\mathrm{x}<3$ or $\mathrm{x}<3 \Rightarrow \mathrm{x}<5$, demand a little more machinery. You can turn such a proposition into one without a free variable by substituting some value for the variable. Thus if $P(x)$ is $x<3$ then $P(5)$ is $5<3=$ false. To get rid of the free variable without substituting a value for it, you can take the 'and' or 'or' of the proposition for all the possible values of the free variable. These have special names and notation ${ }^{6}$ :

$$
\forall \mathrm{x} \mid \mathrm{P}(\mathrm{x})=\mathrm{P}(\mathrm{x} 1) \wedge \mathrm{P}(\mathrm{x} 2) \wedge \ldots \quad \text { for all } \mathrm{x}, \mathrm{P}(\mathrm{x}) . \text { In Spec, }
$$

$$
\text { (ALL } x \mid P(x)) \text { or } \wedge:\{x \mid P(x)\}
$$

${ }^{5}$ Thus $(a=b)=(a \Rightarrow b \wedge b \Rightarrow a)$, which is why $a=b$ is sometimes pronounced " $a$ if and only if $b$ " and written " $a$ iff b".

no agreement on what symbol should separate the $\forall \mathrm{x}$ or $\exists \mathrm{x}$ from the $\mathrm{P}(\mathrm{x})$. We use 'l' here as Spec does, but other people use '.' or '?' or just a space, or write $(\forall x)$ and $(\exists x)$. Logicians traditionally write $(x)$ and $(\exists x)$. 
$\exists \mathrm{x} \mid \mathrm{P}(\mathrm{x})=\mathrm{P}(\mathrm{x} 1) \vee \mathrm{P}(\mathrm{x} 2) \vee \ldots \quad$ there exists an $\mathrm{x}$ such that $\mathrm{P}(\mathrm{x})$. In Spec,

(EXISTS $x \mid P(x))$ or $\vee:\{x \mid P(x)\}$

Here the xi range over all the possible values of the free variables. ${ }^{7}$ The first is called 'universal quantification'; as you can see, it corresponds to conjunction. The second is called 'existential quantification' and corresponds to disjunction. If you remember this you can easily figure out what the quantifiers do with respect to the other operators.

In particular, DeMorgan's laws generalize to quantifiers:

$$
\begin{aligned}
& \sim(\forall x \mid P(x))=(\exists x \mid \sim P(x)) \\
& \sim(\exists x \mid P(x))=(\forall x \mid \sim P(x))
\end{aligned}
$$

Also, because $\wedge$ and $\vee$ are conjunctive and therefore monotonic, $\forall$ and $\exists$ are conjunctive and therefore monotonic.

It is not true that you can reverse the order of $\forall$ and $\exists$, but it's sometimes useful to know that having $\exists$ first is stronger:

$$
\exists \mathrm{y}|\forall \mathrm{x}| \mathrm{P}(\mathrm{x}, \mathrm{y}) \Rightarrow \forall \mathrm{x}|\exists \mathrm{y}| \mathrm{P}(\mathrm{x}, \mathrm{y})
$$

Intuitively this is clear: $\mathrm{a} y$ that works for every $\mathrm{x}$ can surely do the job for each particular $\mathrm{x}$.

If we think of $\mathrm{P}$ as a relation, the consequent in this formula says that $\mathrm{P}$ is total (relates every $\mathrm{x}$ to some y). It doesn't tell us anything about how to find a y that is related to $\mathrm{x}$. As computer scientists, we like to be able to compute things, so we prefer to have a function that computes $y$, or the set of y's, from $x$. This is called a 'Skolem function'; in Spec you write P. func (or P . setF for the set). $P$. func is total if $P$ is total. Or, to turn this around, if we have a total function $f$ such that $\forall x \mid P(x, f(x))$, then certainly $\forall x|\exists y| P(x, y)$; in fact, $y=f(x)$ will do Amazing.

${ }^{7}$ In general this might not be a countable set, so the conjunction and disjunction are written in a somewhat misleading way, but this complication won't make any difference to us.

\section{Summary of logic}

The $\wedge$ and $\vee$ operators are commutative and associative and distribute over each other.

DeMorgan's laws: $\sim(a \wedge b)=\sim a \vee \sim b$

$$
\sim(a \vee b)=\sim a \wedge \sim b
$$

Implication: $\quad(\mathrm{a} \Rightarrow \mathrm{b})=\sim \mathrm{a} \vee \mathrm{b}$

Implication is the ordering in a lattice (a partially ordered set in which every subset has a least upper and a greatest lower bound) with

$\begin{array}{llll}\text { top } & =\text { false } & & \text { so false } \Rightarrow \text { true } \\ \text { bottom } & =\text { true } & & \\ \text { meet } & =\wedge & & \text { least upper bound, so }(a \wedge b) \Rightarrow a \\ \text { join } & =\vee & & \text { greatest lower bound, so } a \Rightarrow(a \vee b)\end{array}$

For all $x, P(x)$ :

$$
\forall \mathrm{x} \mid \mathrm{P}(\mathrm{x})=\mathrm{P}(\mathrm{x} 1) \wedge \mathrm{P}(\mathrm{x} 2) \wedge \ldots
$$

There exists an $x$ such that $P(x)$ :

$$
\exists x \mid P(x)=P(x 1) \vee P(x 2) \vee \cdots
$$

Index for logic

$\sim, 6$
$==>, 6$
$\Rightarrow, 6$
ALL, 9
and, 6
antecedent, 6
Anti-symmetric, 8
associative, 6
bottom, 8
commutative, 6
conjunction, 6
conjunctive, 6
consequent, 6
contrapositive, 8
DeMorgan's laws, 7,9
disjunction, 6
disjunctive, 6
distribute, 6
existential quantification, 9
EXISTS, 9
follows from, 7
free variables, 8
greatest lower bound, 8
if a then b, 7
implication, 6,7
join, 8
lattice, 8
least upper bound, 8

meet, 8

monotonic, 8

negation, 6

not, 6

only if, 7

operators, 6

or, 6

ordering on Bool, 7

predicate logic, 8

propositions, 6

propositions, 6

quantifiers,

Skolem function, 9

stronger than, 7

top, 8

transitive, 8

truth table, 6

universal quantification, 9

weaker than, 7 



\section{Introduction to Spec}

This handout explains what the Spec language is for, how to use it effectively, and how it differs from a programming language like C, Pascal, Clu, Java, or Scheme. Spec is very different from these languages, but it is also much simpler. Its meaning is clearer and Spec programs are more succinct and less burdened with trivial details. The handout also introduces the main constructs that are likely to be unfamiliar to a programmer. You will probably find it worthwhile to read it over more than once, until those constructs are familiar.

Spec is a language for writing precise descriptions of digital systems, both sequential and concurrent. In Spec you can write something that differs from a practical implementation (for instance, one written in C) only in minor details of syntax. This sort of thing is usually called a program. Or you can write a very high level description of the behavior of a system, usually called a specification. A good specification is almost always quite different from a good program. You can use Spec to write either one, but not the same style of Spec. The flexibility of the language means that you need to know the purpose of your Spec in order to write it well.

Most people know a lot more about writing programs than about writing specs, so this introduction emphasizes how Spec differs from a programming language and how to use it to write good specs. It does not attempt to be either complete or precise, but other handouts fill these needs. The Spec Reference Manual (handout 4) describes the language completely; it gives the syntax of Spec precisely and the semantics informally. Atomic Semantics of Spec (handout 9) describes precisely the meaning of an atomic command; here 'precisely' means that you should be able to get an unambiguous answer to any question. The section "Non-Atomic Semantics of Spec" in handout 17 on formal concurrency describes the meaning of a non-atomic command.

Spec's notation for commands, that is, for changing the state, is derived from Edsger Dijkstra's guarded commands (E. Dijkstra, A Discipline of Programming, Prentice-Hall, 1976) as extended by Greg Nelson (G. Nelson, A generalization of Dijkstra's calculus, ACM TOPLAS 11, 4, Oct. 1989, pp 517-561). The notation for expressions is derived from mathematics.

This handout starts with a discussion of specifications and how to write them, with many small examples of Spec. Then there is an outline of the Spec language, followed by three extended examples of specs and implementations. At the end are two handy tear-out one-page summaries, one of the language and one of the official POCS strategy for writing specs and implementations.

\section{What is a specification for?}

The purpose of a specification is to communicate precisely all the essential facts about the behavior of a system. The important words in this sentence are:

communicate The spec should tell both the client and the implementer what each needs to know.

precisely We should be able to prove theorems or compile machine instructions based on the spec.

essential Unnecessary requirements in the spec may confuse the client or make it more expensive to implement the system.

behavior We need to know exactly what we mean by the behavior of the system.

Communication

Spec mediates communication between the client of the system and its implementer. One way to view the specification is as a contract between these parties:

The client agrees to depend only on the system behavior expressed in the spec; in return it can count on the implementation to provide a system that actually does behave as the spec says it should.

The implementer agrees to provide a system that behaves according to the spec; in return it is free to arrange the internals of the system however it likes, and it does not have to deliver anything not laid down in the spec.

Usually the implementer of a spec is a programmer, and the client is another programmer. Usually the implementer of a program is a compiler or a computer, and the client is a programmer.

\section{Behavior}

What do we mean by behavior? In real life a spec defines not only the functional behavior of the system, but also its performance, cost, reliability, availability, size, weight, etc. In this course we will deal with these matters informally if at all. The Spec language doesn't help much with them.

Spec is concerned only with the possible state transitions of the system, on the theory that the possible state transitions tell the complete story of the functional behavior of a digital system. So we make the following definitions:

A state is the values of a set of names (for instance, $x=3$, color $=r e d$ ).

A history is a sequence of states such that each pair of adjacent states is a transition of the system (for instance, $x=1 ; \quad x=2 ; \quad x=5$ is the history if the initial state is $x=1$ and the transitions are "if $x=1$ then $x:=x+1$ " and "if $x=2$ then $x:=2 * x+1$ ").

A behavior is a set of histories (a non-deterministic system can have more than one history). 
How can we specify a behavior?

One way to do this is to just write down all the histories in the behavior. For example, if the state just consists of a single integer, we might write

$$
\begin{aligned}
& \begin{array}{llllllllllllllllll}
1 & 1 & 1 & 1 & 1 & 1 & 1 & 1 & 1 & 1 & 1 & 1 & 1 & 1 & 1 & 1 & 1 & 1 \\
1 & 2 & 1 & 1 & 1 & 1 & 1 & 1 & 1 & 1 & 1 & 1 & 1 & 1 & 1 & 1 & 1 & 1
\end{array}
\end{aligned}
$$

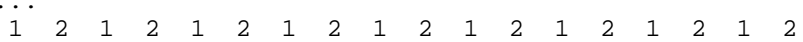

$$
\begin{aligned}
& \begin{array}{llllllllllllllllll}
\cdots & 2 & 3 & 4 & 5 & 1 & 2 & 3 & 1 & 2 & 3 & 4 & 5 & 6 & 7 & 8 & 9 & 10
\end{array}
\end{aligned}
$$

The example reveals two problems with this approach:

The sequences are long, and there are a lot of them, so it takes a lot of space to write them down. In fact, in most cases of interest the sequences are infinite, so we can't actually write them down.

It isn't too clear from looking at such a set of sequences what is really going on.

Another description of this set of sequences from which these examples are drawn is "18 integers, each one either 1 or one more than the preceding one." This is concise and understandable, but it is not formal enough either for mathematical reasoning or for directions to a computer.

Precise

In Spec the set of sequences can be described in many ways, for example, by the expression

$$
\left\{\begin{aligned}
\text { s: SEQ Int } \mid & \text { s.size }=18 \\
& 八 \text { (ALL } i: \text { Int } 0<=i 八 i<\text { s.size }==>
\end{aligned}\right.
$$$$
s(i)=1 \backslash(i>0 / N(i)=s(i-1)+1))
$$

Here the expression in $\{\ldots\}$ is very close to the usual mathematical notation for defining a set. Read it as "The set of all s which are sequences of integers such that s. size $=18$ and ...". Spec sequences are indexed from 0 . The (ALL ...) is a universally quantified predicate, and $==>$ stands for implication, since Spec uses the more familiar $=>$ for 'then' in a guarded command. Throughout Spec the 'I' symbol separates a declaration of some new names and their types from the scope in which they are meaningful.

Alternatively, here is a state machine that generates the sequences we want as the successive values of the variable $i$. We specify the transitions of the machine by starting with primitive assignment commands and putting them together with a few kinds of compound commands. Each command specifies a set of possible transitions.

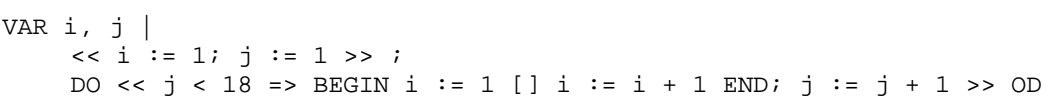

Here there is a good deal of new notation, in addition to the familiar semicolons, assignments, and plus signs.
VAR $i, j \mid$ introduces the local variables $i$ and $j$ with arbitrary values. Because ; binds more tightly than $\mid$, the scope of the variables is the rest of the example.

The $<<\ldots$. > brackets delimit the atomic actions or transitions of the state machine. All the changes inside these brackets happen as one transition of the state machine.

$j<18 \Rightarrow \ldots$ is a transition that can only happen when $j<18$. Read it as "if $j<18$ then ...". The $j<18$ is called a guard. If the guard is false, we say that the entire command fails.

$i:=1[$ [ $\quad:=i+1$ is a non-deterministic transition which can either set $i$ to 1 or increment it. Read [] as 'or'.

The BEGIN ... END brackets are just brackets for commands, like $\{\ldots\}$ in $\mathrm{C}$. They are there because $=>$ binds more tightly than the [ ] operator inside the brackets; without them the meaning would be "either set $i$ to 1 if $j<18$ or increment $i$ and $j$ unconditionally".

Finally, the $D O \ldots$ OD brackets mean: repeat the ... transition as long as possible. Eventually $j$ becomes 18 and the guard becomes false, so the command inside the DO ... OD fails and can no longer happen.

The expression approach is better when it works naturally, as this example suggests, so Spec has lots of facilities for describing values: sequences, sets, and functions as well as integers and booleans. Usually, however, the sequences we want are too complicated to be conveniently described by an expression; a state machine can describe them much more easily.

State machines can be written in many different ways. When each transition involves only simple expressions and changes only a single integer or boolean state variable, we think of the state machine as a program, since we can easily make a computer exhibit this behavior. When there are transitions that change many variables, non-deterministic transitions, big values like sequences or functions, or expressions with quantifiers, we think of the state machine as a specification, since it may be much easier to understand and reason about it, but difficult to make a computer exhibit this behavior. In other words, large atomic actions, non-determinism, and expressions that compute sequences or functions are hard to implement. It may take a good deal of ingenuity to find an implementation that has the same behavior but uses only the small, deterministic atomic actions and simple expressions that are easy for the computer.

Essential

The hardest thing for most people to learn about writing specs is that $a$ spec is not a program. A spec defines the behavior of a system, but unlike a program it need not, and usually should not, give any practical method for producing this behavior. Furthermore, it should pin down the behavior of the system only enough to meet the client's needs. Details in the spec that the client doesn't need can only make trouble for the implementer.

The example we just saw is too artificial to illustrate this point. To learn more about the difference between a spec and an implementation consider the following: 
CONST eps $:=10 * *-8$

APROC SquareRoot0 ( $x$ : Real) $\rightarrow$ Real $=$

$$
\text { << VAR y : Real | Abs }\left(x-y^{\star} y\right)<\text { eps } \Rightarrow \text { RET y >> }
$$

(Spec as described in the reference manual doesn't have a Real data type, but we'll add it for the purpose of this example.)

The combination of VAR and $=>$ is a very common Spec idiom; read it as "choose a y such that Abs $\left(x-y^{*} y\right)<$ eps and do RET y". Why is this the meaning? The VAR makes a choice of any Real as the value of $y$, but the entire transition on the second line cannot occur unless the guard is true. The result is that the choice is restricted to a value that satisfies the guard.

What can we learn from this example? First, the result of squareRoot $0(x)$ is not determined by the value of $x$; any result whose square is within eps of $x$ is possible. This is why SquareRoot 0 is written as a procedure rather than a function; the result of a function has to be determined by the arguments and the current state, so that the value of an expression like $f(x)=f(x)$ will be true. In other words, SquareRoot 0 is non-deterministic.

Why did we write it that way? First of all, there might not be any Real (that is, any floating-point number of the kind used to represent Real) whose square exactly equals x. ${ }^{1}$ Second, we may not want to pay for an implementation that gives the closest possible answer. Instead, we may settle for a less accurate answer in the hope of getting the answer faster.

You have to make sure you know what you are doing, though. This spec allows a negative result, which is perhaps not what we really wanted. We could have written (highlighting changes with boxes):

APROC SquareRoot1 $(x$ : Real) $\rightarrow$ Real $=$

< VAR y : Real | y > $>$ / Abs $\left(\mathrm{x}-\mathrm{y}^{\star} \mathrm{y}\right)<$ eps $=>\operatorname{RET} \mathrm{y}>>$

to rule that out. Also, the spec produces no result if $x<0$, which means that SquareRoot $1(-1)$ will fail (see the section on commands for a discussion of failure). We might prefer a total function that raises an exception:

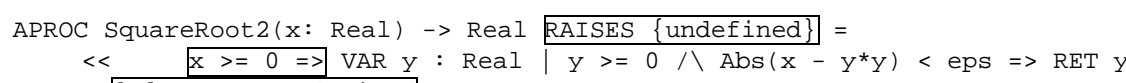

The $\left[{ }^{*}\right]$ is 'else'; it does its second operand iff the first one fails. Exceptions in Spec are much like exceptions in CLU. An exception is contagious: once started by a RAISE it causes any containing expression or command to yield the same exception, until it runs into an exception handler (not shown here). The RAISES clause of a routine declaration must list all the exceptions that the procedure body can generate, either by RAISES or by invoking another routine.

An implementation of this spec would look quite different from the spec itself. Instead of the existential quantifier implied by the VAR $y$, it would have an algorithm for finding $y$, for

${ }^{1}$ We could accommodate this fact of life by specifying the closest floating-point number. This would still be nondeterministic in the case that two such numbers are equally close, so if we wanted a deterministic spec we would have to give a rule for choosing one of them, for instance, the smaller. instance, Newton's method. In the algorithm you would only see operations that have obvious implementations in terms of the load, store, arithmetic, and test instructions of a computer. Probably the implementation would be deterministic.

Another way to write these specs is as functions that return the set of possible answers. Thus FUNC SquareRoots1 $(x$ : Real) $\rightarrow$ SET Real $=$

$$
\text { RET }\left\{y: \text { Real } \mid \mathrm{y}>=0 \text { 八 Abs }\left(\mathrm{x}-\mathrm{y}^{*} \mathrm{y}\right)<\mathrm{eps}\right\}
$$

Note that the form inside the $\{\ldots\}$ set constructor is the same as the guard on the RET. To get a single result you can use the set's choose method: SquareRoots1 (2). choose. ${ }^{2}$

In the next section we give an outline of the Spec language. Following that are three extended examples of specs and implementations for fairly realistic systems. At the end is a one-page summary of the language.
${ }^{2} \mathrm{r}:=$ SquareRoots $1(\mathrm{x})$. choose (using the function) is almost the same as $\mathrm{r}:=$ SquareRoot1 $(\mathrm{x})$ (using the procedure). The difference is that because choose is a function it always returns the same element (even though we argument. The procedure, on the other hand, is non-deterministic and can return different values on successive calls. 


\section{An outline of the Spec language}

The Spec language has two main parts:

- An expression describes how to compute a result (a value or an exception) as a function of other values: either literal constants or the current values of state variables.

- A command describes possible transitions of the state variables. Another way of saying this is that a command is a relation on states: it allows a transition from s1 to s2 iff it relates s1 to s.

Both are based on the state, which in Spec is a mapping from names to values. The names are called state variables or simply variables: in the sequence example above they are $i$ and $j$.

Actually a command relates states to outcomes; an outcome is either a state (a normal outcome) or a state together with an exception (an exceptional outcome).

There are two kinds of commands:

- An atomic command describes a set of possible transitions, or equivalently, a set of pairs of states. For instance, the command $<<:=i+1>$ describes the transitions $i=1 \rightarrow i=2$, $i=2 \rightarrow i=3$, etc. (Actually, many transitions are summarized by $i=1 \rightarrow i=2$, for instance, $\quad(i=1$, $j=1) \rightarrow(i=2, j=1)$ and $(i=1, j=15) \rightarrow(i=2, \quad j=15))$. If a command allows more than one transition from a given state we say it is non-deterministic. For instance, on page 3 the command BEGIN $i:=1$ [] $i:=i+1$ END allows the transitions $i=2 \rightarrow i=1$ and $i=2 \rightarrow i=3$.

- A non-atomic command describes a set of sequences of states (by contrast with the set of pairs for an atomic command). More on this below.

A sequential program, in which we are only interested in the initial and final states, can be described by an atomic command.

The meaning of an expression, which is a function from states to values (or exceptions), is much simpler than the meaning of an atomic command, which is a relation between states, for two reasons:

- The expression yields a single value rather than an entire state.

- The expression yields at most one value, whereas a non-deterministic command can yield many final states.

A atomic command is still simple, much simpler than a non-atomic command, because:

- Taken in isolation, the meaning of a non-atomic command is a relation between an initial state and a history. Again, many histories can stem from a single initial state.

- The meaning of the composition of two non-atomic commands is not any simple combination of their relations, such as the union, because the commands can interact if they share any variables that change.
These considerations lead us to describe the meaning of a non-atomic command by breaking it down into its atomic subcommands and connecting these up with a new state variable called a program counter. The details are somewhat complicated; they are sketched in the discussion of atomicity below, and described in handout 17 on formal concurrency.

The moral of all this is that you should use the simpler parts of the language as much as possible: expressions rather than atomic commands, and atomic commands rather than non-atomic ones.

To encourage this style, Spec has a lot of syntax and built-in types and functions that make it easy to write expressions clearly and concisely. You can write many things in a single Spec expression that would require a number of $\mathrm{C}$ statements, or even a loop. Of course, an implementation with a lot of concurrency will necessarily have more non-atomic commands, but this complication should be put off as long as possible.

Organizing the program

In addition to the expressions and commands that are the core of the language, Spec has four other mechanisms that are useful for organizing your program and making it easier to understand.

- A routine is a named computation with parameters, in other words, an abstraction of the computation. Parameters are passed by value. There are four kinds of routine:

A function (defined with FUNC) is an abstraction of an expression.

An atomic procedure (defined with APROC) is an abstraction of an atomic command.

A general procedure (defined with PROC) is an abstraction of a non-atomic command.

A thread (defined with THREAD) is the way to introduce concurrency.

- A type is a highly stylized assertion about the set of values that a name or expression can assume. A type is also a convenient way to group and name a collection of routines, called its methods, that operate on values in that set.

- An exception is a way to report an unusual outcome.

- A module is a way to structure the name space into a two-level hierarchy. An identifier i declared in a module $\mathrm{m}$ has the name $\mathrm{m}$. $i$ throughout the program. A class is a module that can be instantiated many times to create many objects.

A Spec program is some global declarations of variables, routines, types, and exceptions, plus a set of modules each of which declares some variables, routines, types, and exceptions.

The next two sections describe things about Spec's expressions and commands that may be new to you. It doesn't answer every question about Spec; for those answers, read the reference manual and the handouts on Spec semantics. There is a one-page summary at the end of this handout. 


\section{Expressions, types, and functions}

Expressions are for computing functions of the state. A Spec expression is a constant, a variable, or an invocation of a function on an argument that is some sub-expression. The values of these expressions are the constant, the current value of the variable, or the value of the function at the value of the argument. There are no side-effects; those are the province of commands. There is quite a bit of syntactic sugar for function invocations. An expression may be undefined in a state; if a simple command evaluates an undefined expression, the command fails (see below).

A Spec type defines two things:

A set of values; we say that a value has the type if it's in the set. The sets are not disjoint.

A set of functions called the methods of the type. There is convenient syntax v . m for invoking method $\mathrm{m}$ on a value $\mathrm{v}$ of the type.

Spec is strongly typed. This means that you are supposed to declare the types of your variables, just as you do in Pascal or CLU. In return the language defines a type for every expression ${ }^{3}$ and ensures that the value of the expression always has that type. In particular, the value of a variable always has the declared type. You should think of a type declaration as a stylized comment that has a precise meaning and could be checked mechanically.

If Foo is a type, you can omit it in a declaration of the identifiers foo, foo1, foo' etc. Thus VAR int1, bool2, char'' | ...

is short for

VAR int1: Int, bool2: Bool, char'': Char $\mid \ldots$

Spec has the usual types: Int, Nat (non-negative Int), Bool, functions, sets, records, tuples, and variable-length arrays called sequences. A sequence is a function whose domain is $\{0,1, \ldots$, $\mathrm{n}-1$ \} for some $\mathrm{n}$. In addition to the usual functions like "+" and " $\backslash /$ ", Spec also has some less usual operations on these types, which are valuable when you want to suppress implementation detail: constructors and combinations.

You can make a type with fewer values using suchthAт. For example,

TYPE $\mathrm{T}=$ Int SUCHTHAT ( $i$ : Int $\mid 0<=i / i<=4)$

has the value set $\{0,1,2,3,4\}$. Here the $(\backslash \ldots)$ is a lambda expression that defines a function from Int to Bool, and a value has type $\mathrm{T}$ if it's an Int and the function maps it to true.

Section 5 of the reference manual describes expressions and lists all the built-in operators. You should read the list, which also gives their precedence and has pointers to explanations of their meaning. Section 4 describes the types. Section 9 defines the built-in methods for sequences, sets, and functions; you should read it over so that you know the vocabulary.

${ }^{3}$ Note that a value may have many types, but a variable or an expression has exactly one type: for a variable, it's the declared type, and for a complex expression it's the result type of the top-level function in the expression.

\section{Constructors}

Constructors for functions, sets, and sequences make it easy to toss large values around. For instance, you can describe a database as a function db from names to data records with two fields:

TYPE DB $=($ String $\rightarrow$ Entry)

TYPE Entry $=[$ salary: Int, birthdate: Int$$
\operatorname{VAR} d \mathrm{db}:=\mathrm{DB}\{\}
$$

Here $\mathrm{db}$ is initialized using a function constructor whose value is a function undefined everywhere. The type can be omitted in a variable declaration when the variable is initialized; it is taken to be the type of the initializing expression. The type can also be omitted when it is the upper case version of the variable name, DB in this example.

Now you can make an entry with

$\mathrm{db}:=\mathrm{db}\{$ "Smith" $\rightarrow$ Entry $\{$ salary $:=23000$, birthdate $:=1955\}$ using another function constructor. The value of the constructor is a function that is the same as $\mathrm{db}$ except at the argument "Smith", where it has the value Entry $\{\ldots\}$, which is a record constructor. The assignment could also be written

$\mathrm{db}($ "Smith") $:=$ Entry\{salary $:=23000$, birthdate $:=1955\}$

which changes the value of the db function at "Smith" without changing it anywhere else. This is actually a shorthand for the previous assignment. You can omit the field names if you like, so that

$\mathrm{db}($ "Smith") $:=$ Entry $\{23000,1955\}$

has the same meaning as the previous assignment. Obviously this shorthand is less readable and more error-prone, so use it with discretion. Another way to write this assignment is

db ("Smith"). salary $:=23000 ;$ db ("Smith") .birthdate $:=1955$

The set of names in the database can be expressed by a set constructor. It is just $\{n$ : String $\mid \mathrm{db} ! n\}$,

in other words, the set of all the strings for which the db function is defined ('!' is the 'isdefined' operator; that is, $f ! x$ is true iff $f$ is defined at $x$ ). Read this "the set of strings $n$ such that $\mathrm{db} ! \mathrm{n}$ ". You can also write it as $\mathrm{db}$. dom, the domain of $\mathrm{db}$; section 9 of the reference manual defines lots of useful built in methods for functions, sets, and sequences. It's important to realize that you can freely use large (possibly infinite) values such as the db function. You are writing a specification, and you don't need to worry about whether the compiler is clever enough to turn an expensive-looking manipulation of a large object into a cheap incremental update. That's the implementer's problem (so you may have to worry about whether she is clever enough).

If we wanted the set of lengths of the names, we would write

$\{\mathrm{n}$ : String $|\mathrm{db} ! \mathrm{n}| \mathrm{n}$.size $\}$

This three part set constructor contains $i$ if and only if there exists an $n$ such that $d b ! n$ and $i=n$.size. So $\{n$ : String $\mid d b ! n\}$ is short for $\{n$ : String $|d b ! n| n\}$. You can introduce more than one name, in which case the third part defaults to the last name. For example, if we represent a directed graph by a function on pairs of nodes that returns true when there's an edge from the first to the second, then

\{n1: Node, n2: Node | graph(n1, n2) | n2\} is the set of nodes that are the target of an edge, and the "| n2" could be omitted.

Following standard mathematical notation, you can also write 
\{f : IN openfiles | f.modified $\}$

to get the set of all open, modified files. This is equivalent to

$\{f:$ File | $\mathrm{f}$ IN openFiles $八$ f.modified

because if $s$ is a SET T, then IN $s$ is a type whose values are the T's in $s$; in fact, it's the type T SUCHтнAт ( $\backslash t \mid t$ IN $s$ ). This form also works for sequences, where the second operand of : IN provides the ordering. So if $s$ is a sequence of integers, $\{x:$ IN $s \mid x>0\}$ is the positive ones, $\{\mathrm{x}:$ IN $\mathrm{s}|\mathrm{x}>0| \mathrm{x} * \mathrm{x}\}$ is the squares of the positive ones, and $\{\mathrm{x}$ : IN $\mathrm{s}$

||$x * x\}$ is the squares of all the integers, because an omitted predicate defaults to true. ${ }^{4}$

To get sequences that are more complicated you can use sequence generators with BY and WHILE. $\{i:=1$ BY $i+1$ WHILE $i<=5 \mid$ true $\mid i\}$

is $\{1,2,3,4,5\}$; the second and third parts could be omitted. This is just like the "for" construction in C. An omitted WHILE defaults to true, and an omitted := defaults to an arbitrary choice for the initial value. If you write several generators, each variable gets a new value for each value produced, but the second and later variables are initialized first. So to get the sums of successive pairs of elements of $s$, write

$\{x:=s$ BY x.tail WHILE $x$.size $>1 \mid x(0)+x(1)\}$

To get the sequence of partial sums of $s$, write (eliding || sum at the end)

$\{\mathrm{x}:$ IN $\mathrm{s}$, sum $:=0$ BY sum $+\mathrm{x}\}$

Taking last of this would give the sum of the elements of s. To get a sequence whose elements are reversed from those of $s$, write

$\{x: I N$ s, rev $:=\{\}$ BY $\{x\}+$ rev $\}$.last

To get the sequence $\{f(e), f 2(e), \ldots, f n(e)\}$, write

$\{\mathrm{i}:$ IN $1 \ldots \mathrm{n}$, iter $:=\mathrm{e}$ BY $\mathrm{f}($ iter $)\}$

This uses the $\ldots$ operator; $i \ldots j$ is the sequence $\{i, i+1, \ldots, j-1, j\}$.

\section{Combinations}

A combination is a way to combine the elements of a sequence or set into a single value using an infix operator, which must be associative, must have an identity, and must be commutative if it is applied to a set. You write "operator : sequence or set". Thus

+ : (SEQ String) $\{$ "He", "l", "lo" $\}=$ "He" + "l" + "lo" = "Hello"

because + on sequences is concatenation, and

$$
+:\{i: \operatorname{IN} 1 \ldots 4|| i * \star 2\}=1+4+9+16=30
$$

Existential and universal quantifiers make it easy to describe properties without explaining how to test for them in a practical way. For instance, a predicate that is true iff the sequence $s$ is sorted is

(ALL $i$ : IN 1 ... s.size-1 $\mid s(i-1)<=s(i)$ )

This is a common idiom; read it as

"for all $i$ in 1 .. s.size-1, s(i-1) $<=s(i)$ ".

This could also be written

(ALL $i$ : IN (s.dom - \{0\}) $\mid s(i-1)<=s(i))$

since $\mathrm{s}$.dom is the domain of the function $\mathrm{s}$, which is the non-negative integers $<\mathrm{s}$. size.

${ }^{4}$ In the sequence form, IN $\mathrm{s}$ is not a type but a special construct; treating it as a type would throw away the essential ordering information.
Because a universal quantification is just the conjunction of its predicate for all the values of the bound variables, it is simply a combination using $八$ as the operator:

$$
\text { (ALL } \quad \text { i } \mid \text { Predicate (i)) }=八:\{i \mid \text { Predicate }(i)
$$

Similarly, an existential quantification is just a similar disjunction, hence a combination using $\backslash$ as the operator:

(EXISTS $i \mid$ Predicate(i)) $=\backslash /:\{$ i | Predicate(i) $\}$

Spec has the redundant ALL and EXISTS notations because they are familiar.

If you want to get your hands on a value that satisfies an existential quantifier, you can construct the set of such values and use the choose method to pick out one of them

$$
\text { \{i | Predicate(i) \}. choose }
$$

This is deterministic: choose always returns the same value given the same set (a necessary property for it to be a function). It is undefined if the set is empty, which is the case in the example if no $i$ satisfies Predicate.

The VAR command described in the next section on commands is another form of existential quantification that lets you get your hands on the value, but it is non-deterministic.

\section{Functions}

Like everything (except types), functions are ordinary values in Spec. Given a function, you can use a function constructor to make another one that is the same except at a particular argument, as in the DB example above. Another example is $f\{x \rightarrow 0\}$, which is the same as $f$ except that it is 0 at $x$. If you have never seen a construction like this one, think about it for a minute. Suppose you had to implement it. If $f$ is represented as a table of (argument, result) pairs, the implementation will be easy. If $f$ is represented by code that computes the result, the implementation is less obvious, but you can make a new piece of code that says

$$
\left.\left(\backslash \mathrm{y}: \operatorname{Int} \mid\left((\mathrm{y}=\mathrm{x})=>0{ }^{*}\right] \mathrm{f}(\mathrm{y})\right)\right)
$$

Here ' $'$ ' is 'lambda', and the subexpression $\left((y=x) \Rightarrow 0\left[{ }^{*}\right] f(y)\right)$ is a conditional, modeled on the conditional commands we saw in the first section; its value is 0 if $y=x$ and $f(y)$ otherwise, so we have changed $f$ just at 0 , as desired. If the else clause $\left[{ }^{*}\right] f_{(y)}$ is omitted, the condition is undefined if $\mathrm{y} \# \mathrm{x}$. Of course in a running program you probably wouldn't want to construct new functions very often, so a piece of Spec that is intended to be close to a practical implementation must use function constructors carefully.

Functions can return functions as results. Thus $\mathrm{T}->\mathrm{U}->\mathrm{V}$ is the type of a function that takes an and returns a function of type $U->V$, which in turn takes a $U$ and returns a $V$. If $f$ has this type, then $f(t)$ has type $U->V$, and $f(t)(u)$ has type $V$. Compare this with $(T, U) \rightarrow V$, the type of a function which takes an $\mathrm{I}$ and $\mathrm{a} U$ and returns a $\mathrm{V}$. If $g$ has this type, $g(t)$ doesn't type-check and $g(t, u)$ has type $v$. Obviously $f$ and $g$ are closely related, but they are not the same.

You can define your own functions either by lambda expressions like the one above, or more generally by function declarations like this one

$$
\text { FUNC NewF }(y \text { : Int }) \rightarrow \text { Int }=\operatorname{RET}\left((y=x) \Rightarrow 0\left[{ }^{*}\right] f(y)\right)
$$

The value of this NewF is the same as the value of the lambda expression. To avoid some redundancy in the language, the meaning of the function is defined by a command in which RET sub-commands specify the value of the function. The command might be syntactically nondeterministic (for instance, it might contain VAR or []), but it must specify at most one result 
value for any argument value; if it specifies no result values for an argument or more than one value, the function is undefined there. If you need a full-blown command in a function constructor, you can write it with LAMBDA instead of $\backslash$ :

$$
\left(\operatorname{LAMBDA}(\mathrm{y}: \text { Int }) \rightarrow \operatorname{Int}=\operatorname{RET}\left((\mathrm{y}=\mathrm{x}) \Rightarrow 0\left[{ }^{*}\right] \mathrm{f}(\mathrm{y})\right)\right)
$$

You can compose two functions with the * operator, writing $f * g$. This means to apply $f$ first and then $g$. It is often useful when $f$ is a sequence (remember that a $S E Q T$ is a function from $\{0$, $1, \ldots$, size $\left.^{-1}\right\}$ to $\mathrm{T}$ ), since the result is a sequence with every element of $f$ mapped by $g$. So:$$
(0 \ldots 4) *\{\backslash i \text { : Int } \mid i * i\}=(\operatorname{SEQ} \text { Int })\{0,1,4,9,16\}
$$

since $0 \ldots 4=\{0,1,2,3,4\}$ because Int has a method ... with the obvious meaning: $i \ldots j=\{i, i+1, \ldots, j-1, j\}$. In the section on constructors we saw another way to write

$$
(0 \ldots 4) *\{\backslash i: \text { Int } \mid i * i\} \text {, }
$$

$$
\{i: \text { IN } 0 \text {. }
$$

This is more convenient when the mapping function is defined by an expression, as it is here, but it's less convenient if the mapping function already has a name. Then it's shorter and clearer to write

$(0 \ldots 4) *$ factorial

rather than

$$
\{i: \text { IN } 0 \ldots 4 \mid \text { factorial(i) }\} \text {. }
$$

\section{Methods}

Methods are a convenient way of packaging up some functions with a type so that the functions can be applied to values of that type concisely and without mentioning the type itself. Look at the definitions in section 9 of the Spec Reference Manual, which give methods for the built-in types SEQ T, SET T, and T->U. If $s$ is a SEQ T, $s$.head is Sequence $[T]$. Head $(s)$, which is just $s(0)$ (which is undefined if $s$ is empty). You can see that it's shorter to write s. head. ${ }^{5}$

\section{You can define your own methods by using WITH. For instance, consider}

TYPE Complex = [re: Real, im: Real $]$ WITH $\{"+":=$ Add, mag:=Mag $\}$

Add and Mag are ordinary Spec functions that you must define, but you can now invoke them on a $c$ which is Complex by writing $c+c^{\prime}$ and $c$.mag, which mean Add $\left(c, c^{\prime}\right)$ and Mag (c). You can use existing operator symbols or make up your own; see section 3 of the reference manual for lexical rules. You can also write Complex."+" and complex.mag to denote the functions Add and Mag; this may be convenient if Complex was declared in a different module. Using Add as a method does not make it private, hidden, static, local, or anything funny like that.

When you nest wITH the methods pile up in the obvious way. Thus

TYPE MoreComplex = Complex WITH $\{"-":=$ Sub, mag:=Mag2 $\}$

has an additional method "-", the same "+" as Complex, and a different mag. Many people call this 'inheritance' and 'overriding'.

5 Of course, $s(0)$ is shorter still, but that's an accident; there is no similar alternative for s.tail.

\section{Commands}

Commands are for changing the state. Spec has a few simple commands, and seven operators for combining commands into bigger ones. The main simple commands are assignment and routine invocation. There are also simple commands to raise an exception, to return a function result, and to SKIP, that is, do nothing. If a simple command evaluates an undefined expression, it fails (see below).

The operators on commands are:

- A conditional operator: predicate $=>$ command, read "if predicate then command". The predicate is called a guard.

- Choice operators: c1 [ [ ] c2 and c1 $\left[{ }^{*}\right] \quad$ c2, read 'or' and 'else'.

- Sequencing operators: c1 ; c2 and c1 EXCEPT handler. The handler is a special form of conditional command: exception $=>$ command.

- Variable introduction: VAR id: T | command, read "choose id of type T such that command doesn't fail".

- Loops: DO command OD.

Section 6 of the reference manual describes commands. Atomic Semantics of Spec gives a precise account of their semantics. It explains that the meaning of a command is a relation between a state and an outcome (a state plus an optional exception), that is, a set of possible state-tooutcome transitions.

\section{Conditionals and choice}

The figure below (copied from Nelson's paper) illustrates conditionals and choice with some very simple examples. Here is how they work:

\section{The command}

$\mathrm{p}=>\mathrm{c}$

means to do $\mathrm{c}$ if $\mathrm{p}$ is true. If $\mathrm{p}$ is false this command fails; in other words, it has no outcome. More precisely, if $s$ is a state in which $p$ is false or undefined, this command does not relate $s$ to any outcome.

What good is such a command? One possibility is that $p$ will be true some time in the future, and then the command will have an outcome and allow a transition. Of course this can only happen in a concurrent program, where there is something else going on that can make $\mathrm{p}$ true. Even if there's no concurrency, there might be an alternative to this command. For instance, it might appear in the larger command

$$
\begin{aligned}
& p=c^{\prime} \\
& p^{\prime} \Rightarrow c^{\prime}
\end{aligned}
$$

in which you read [ ] as 'or'. This fails only if each of $p$ and p' is false or undefined. If both are true (as in the 00 state in the south-west corner of the figure), it means to do either $c$ or $c^{\prime}$; the choice is non-deterministic. If $p^{\prime}$ is $\sim p$ then they are never both false, and if $p$ is defined this command is equivalent to 


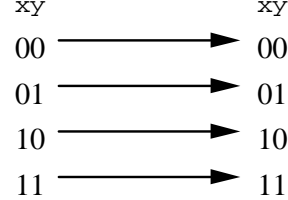

SKIP

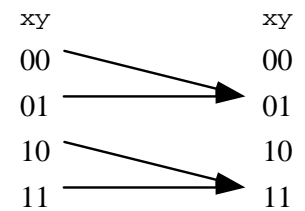

$\mathrm{y}:=1$

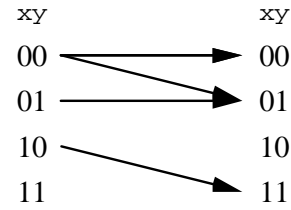

$\mathrm{x}=0 \Rightarrow \operatorname{SKIP}$

[] $\mathrm{y}=0 \Rightarrow \mathrm{y}:=1$

(partial, non-deterministic)

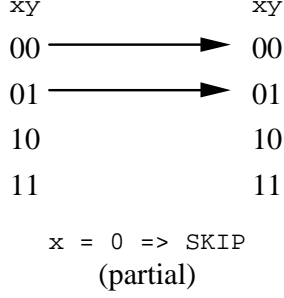

(partial)

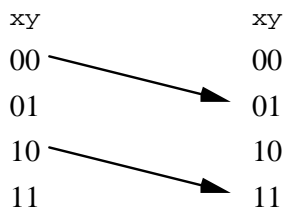

$\mathrm{y}=0 \Rightarrow \mathrm{y}:=1$

(partial)

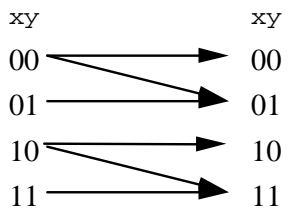

SKIP

[] $\mathrm{y}=0 \Rightarrow \mathrm{y}:=1$ (non-deterministic)

Combining commands

[*] $\begin{aligned} & p \\ & c^{\prime}\end{aligned}=>$

in which you read $\left[^{*}\right]$ as 'else'. On the other hand, if $p$ is undefined the two commands differ, because the first one fails (since neither guard can be evaluated), while the second does $c^{\prime}$.

Both c1 [ ] c2 and c1 [ [*] c2 fail only if both c1 and c2 fail. If you think of a Spec program operationally (that is, as executing one command after another), this means that if the execution makes some choice that leads to failure later on, it must 'back-track' and try the other alternatives until it finds a set of choices that succeed. For instance, no matter what $\mathrm{x}$ is, after $\mathrm{y}=0 \Rightarrow \mathrm{x}:=\mathrm{x}-1 ; \mathrm{x}<\mathrm{y}=>\mathrm{x}:=1$
$\mathrm{y}>0 \Rightarrow \mathrm{x}:=3<\mathrm{y}=>\mathrm{x}:=2$

[*] SKIP if $\mathrm{y}=0$ initially, $\mathrm{x}=1$ afterwards, if $\mathrm{y}>3$ initially, $\mathrm{x}=2$ afterwards, and otherwise $\mathrm{x}$ is unchanged. If you think of it relationally, c1 [ ] c2 has all the transitions of c1 (there are none if $\mathrm{c} 1$ fails, several if it is non-deterministic) as well as all the transitions of $\mathrm{c} 2$. Both failure and non-determinism can arise from deep inside a complex command, not just from a top-level [ ] or VAR.

The precedence rules for commands are

EXCEPT binds tightest

next

$\Rightarrow$ next (for the right operand; the left side is an expression or delimited by VAR)

[ ] [*] bind least tightly.

These rules minimize the need for parentheses, which are written around commands in the ugly form BEGIN ... END or the slightly prettier form IF ... FI; the two forms have the same meaning, but as a matter of style, the latter should only be used around guarded commands. So, for example,

$$
\mathrm{p}=>\mathrm{c} 1 ; \mathrm{c} 2
$$

is the same as

$\mathrm{p}=>$ BEGIN $\mathrm{c} 1 ; \mathrm{c} 2 \mathrm{END}$

and means to do $\mathrm{c} 1$ followed by $\mathrm{c} 2$ if $\mathrm{p}$ is true. To guard only $\mathrm{c} 1$ with $\mathrm{p}$ you must write IF $\left.\mathrm{p} \Rightarrow \mathrm{c} 1 \mathrm{[}^{*}\right]$ SKIP $\mathrm{FI}$; $\mathrm{c} 2$

which means to do $c 1$ if $p$ is true, and then to do $c 2$. The [*] SKIP ensures that the command before the "; " does not fail, which would prevent $c 2$ from getting done. Without the ${ }^{*}{ }^{*}$ SKIP, that is in

IF $\mathrm{p} \Rightarrow \mathrm{C} 1 \mathrm{FI} ; \mathrm{C} 2$

if $p$ is false the IF ... FI fails, so there is no possible outcome from which $c 2$ can be done and the whole thing fails. Thus IF $p \Rightarrow c 1 F I$; $c 2$ has the same meaning as $p \Rightarrow B E G I N c 1 ; c 2$ END, which is a bit surprising.

\section{Sequencing}

A c1 ; c2 command means just what you think it does: first $\mathrm{c} 1$, then $\mathrm{c} 2$. The command $\mathrm{c} 1 ; \mathrm{c} 2$ gets you from state $\mathrm{s} 1$ to state $\mathrm{s} 2$ if there is an intermediate state $\mathrm{s}$ such that $\mathrm{c} 1$ gets you from $s 1$ to $s$ and $c 2$ gets you from $s$ to $s 2$. In other words, its relation is the composition of the relations for $\mathrm{c} 1$ and $\mathrm{c} 2$; sometimes ' $;$ ' is called 'sequential composition'. If $\mathrm{c} 1$ produces an exception, the composite command ignores $\mathrm{c} 2$ and produces that exception.

A c1 EXCEPT ex $\Rightarrow>c 2$ command is just like $c 1$; $c 2$ except that it treats the exception ex the other way around: if $\mathrm{c} 1$ produces the exception ex then it goes on to $\mathrm{c} 2$, but if $\mathrm{c} 1$ produces a normal outcome (or any other exception), the composite command ignores $\mathrm{c} 2$ and produces that outcome.

\section{Variable introduction}

VAR gives you more dramatic non-determinism than []. The most common use is in the idiom VAR $x: T \mid P(x) \Rightarrow c$

which is read "choose some $x$ of type $T$ such that $P(x)$, and do $c$ ". It fails if there is no $x$ for which $P(x)$ is true and $C$ succeeds. If you just write 
VAR $x: T \mid C$

then VAR acts like ordinary variable declaration, giving an arbitrary initial value to $\mathrm{x}$.

Variable introduction is an alternative to existential quantification that lets you get your hands on the bound variable. For instance, you can write

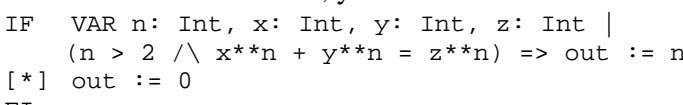

which is read: choose integers $n, x, y, z$ such that $n>2$ and $x^{n}+y^{n}=z^{n}$, and assign $n$ to out; if there are no such integers, assign 0 to out. ${ }^{6}$ The command before the [ ${ }^{*}$ ] succeeds iff

(EXISTS $\mathrm{n}$ : Int, $\mathrm{x}$ : Int, $\mathrm{y}$ : Int, $\mathrm{z}$ : Int $\mid \mathrm{n}>2$ 八 $\mathrm{x}^{\star \star} \mathrm{n}+\mathrm{y}^{\star \star} \mathrm{n}=\mathrm{z}^{\star \star} \mathrm{n}$ ),

but if we wrote that in a guard there would be no way to set out to one of the n's that exist. We could also write

$$
\text { VAR } s:=\left\{\begin{array}{l}
\mathrm{n}: \text { Int, } \mathrm{x}: \text { Int, } \mathrm{y} \text { : Int, } \mathrm{z} \text { : Int } \\
\mathrm{n}>2 八 \mathrm{x}^{\star \star} \mathrm{n}+\mathrm{y}^{\star \star} \mathrm{n}=\mathrm{z}^{\star \star} \mathrm{n} \\
(\mathrm{n}, \mathrm{x}, \mathrm{y}, \mathrm{z})
\end{array}\right.
$$

to construct the set of all solutions to the equation. Then if $s \#\{\}$, s.choose yields a tuple $(n, x, y, z)$ with the desired property.

You can use VAR to describe all the transitions to a state that has an arbitrary relation $\mathrm{R}$ to the current state: VAR $s^{\prime} \mid R\left(s, s^{\prime}\right) \Rightarrow s:=s^{\prime}$ if there is only one state variable $s$.

The precedence of $\mid$ is higher than [], which means that you can string together different VAR commands with [] or [*], but if you want several alternatives within a VAR you have to use BEGIN ... END or IF ... FI. Thus

$\operatorname{VAR} x: T \mid P(x) \Rightarrow c 1$

$$
\text { [] } q=>c 2
$$

is parsed the way it is indented and is the same as

$$
\text { BEGIN VAR } x: T \mid P(x) \Rightarrow c 1 \text { END }
$$

but you must wite the bract

$$
\text { VAR } x: T \text { | }
$$

$$
\begin{aligned}
& \text { IF } P(x) \Rightarrow c 1 \\
& {[] Q(x)=>C 2}
\end{aligned}
$$$$
\mathrm{FI}
$$

which might be formatted more concisely as

$$
\begin{array}{rll}
\operatorname{VAR} x: & T \\
\operatorname{IF} & P(x) \Rightarrow c 1 \\
{[]} & R(x)=>c 2 \quad F I
\end{array}
$$

or even

VAR $x: T \mid \operatorname{IF} P(x) \Rightarrow c 1[] R(x) \Rightarrow c 2$ FI

You are supposed to indent your programs to make it clear how they are parsed.

${ }^{6}$ A correctness proof for an implementation of this spec defied the best efforts of mathematicians between Fermat's time and 1993.

\section{Loops}

You can always write a recursive routine, but often a loop is clearer. In Spec you use DO ... OD for this. These are brackets, and the command inside is repeated as long as it succeeds. When it fails, the repetition is over and the DO ... OD is complete. The most common form is DO $P \Rightarrow C$ OD

which is read "while P is true do c". After this command, P must be false. If the command inside the DO ... OD succeeds forever, the outcome is a looping exception that cannot be handled. Note that this is not the same as a failure, which simply means no outcome at all.

For example, you can zero all the elements of a sequence $s$ with

VAR $i:=0 \mid$ DO $i<$ s.size $\Rightarrow s(i):=0 ; i-:=1$ OD or the simpler form (which also avoids fixing the order of the assignments)

DO VAR $i \mid s(i) \# 0 \Rightarrow s(i):=0$ OD

This is another common idiom: keep choosing an $i$ as long as you can find one that satisfies some predicate. Since $s$ is only defined for $i$ between 0 and s.size-1, the guarded command fails for any other choice of $i$. The loop terminates, since the $s(i):=0$ definitely reduces the number of $i$ 's for which the guard is true. But although this is a good example of a loop, it is bad style; you should have used a sequence method or function composition:

$$
s:=\operatorname{s.fill}(0, \mathrm{~s} . \operatorname{size})
$$

$$
s:=\{x: \text { IN } s|| 0\}
$$

(a sequence just like s except that every element is mapped to 0 ), remembering that Spec makes it easy to throw around big things. Don't write a loop when a constructor will do, because the loop is more complicated to think about. Even if you are writing an implementation, you still shouldn't use a loop here, because it's quite clear how to write C code for the constructor.

To zero all the elements of s that satisfy some predicate $P$ you can write DO VAR i: Int $\mid(s(i) \# 0 八 P(s(i))) \Rightarrow s(i):=0$ OD Again, you can avoid the loop by using a sequence constructor and a conditional expression $\mathrm{s}:=\left\{\mathrm{x}:\right.$ IN $\left.\mathrm{s}||\left(\mathrm{P}(\mathrm{x})=>\left[{ }^{*}\right] \mathrm{x}\right)\right\}$

Atomicity

Each $\langle<\ldots .>>$ command is atomic. It defines a single transition, which includes moving the program counter (which is part of the state) from before to after the command. If a command is not inside $\langle<\ldots>>$, it is atomic only if there's no reasonable way to split it up: SKIP, HAVOC, RET, RAISE. Here are the reasonable ways to split up the other commands:

- An assignment has one internal program counter value, between evaluating the right hand side expression and changing the left hand side variable.

- A guarded command likewise has one, between evaluating the predicate and the rest of the command.

- An invocation has one after evaluating the arguments and before the body of the routine, and another after the body of the routine and before the next transition of the invoking command.

Note that evaluating an expression is always atomic. 


\section{Modules and names}

Spec's modules are very conventional. Mostly they are for organizing the name space of a large program into a two-level hierarchy: module.id. It's good practice to declare everything except a few names of global significance inside a module. You can also declare CONST's, just like VAR's.

MODULE fOO EXPORT $i, j$, Fact $=$

CONST $\mathrm{C}:=1$

VAR $i:=0$

$$
j:=1
$$

FUNC Fact $(\mathrm{n}:$ Int $) \rightarrow$ Int $=$

IF $\mathrm{n}<=1 \Rightarrow \operatorname{RET} 1$

$[*]$ RET $n$ * Fact $(n-1)$

END foo

You can declare an identifier id outside of a module, in which case you can refer to it as id everywhere; this is short for Global.id, so Global behaves much like an extra module. If you declare id at the top level in module $\mathrm{m}$, id is short for m.id inside of $\mathrm{m}$. If you include it in m's EXPORT clause, you can refer to it as m.id everywhere. All these names are in the global state and are shared among all the atomic actions of the program. By contrast, names introduced by a declaration inside a routine are in the local state and are accessible only within their scope.

The purpose of the EXPORT clause is to define the external interface of a module. This is important because module $\mathrm{T}$ implements module $\mathrm{S}$ iff $\mathrm{T}$ 's behavior at its external interface is a subset of s's behavior at its external interface.

The other feature of modules is that they can be parameterized by types in the same style as CLU clusters. The memory systems modules in handout 5 are examples of this.

You can also declare a class, which is a module that can be instantiated many times. The obj class produces a global obj type that has as its methods the exported identifiers of the class plus a new procedure that returns a new, initialized instance of the class. It also produces a ObjMod module that contains the declaration of the obj type, the code for the methods, and a state variable indexed by obj that holds the state records of the objects. For example:

CLASS Stat EXPORT add, mean, variance, reset $=$

$\operatorname{VAR} \mathrm{n}$

$$
\begin{array}{lll}
\text { n } & \text { Int }:=0 \\
\text { sum } & : & \text { Int }:=0
\end{array}
$$$$
\text { sumsq : Int }:=0
$$

PROC add $(i$ : Int $)=n+:=1$; sum $+:=i$; sumsq $+:=i * * 2$

FUNC mean () $\rightarrow$ Int $=$ RET sum $/ \mathrm{n}$

FUNC variance() $\rightarrow$ Int $=$ RET sumsq/n - self.mean**2

$\mathrm{PROC}$ reset ()$=\mathrm{n}:=0 ;$ sum $:=0 ;$ sumsq $:=0$

END Stat

Then you can write
VAR s: Stat | s := s.new(); s.add(x); s.add(y); print(s.variance)

In abstraction functions and invariants we also write obj.n for field $\mathrm{n}$ in obj's state

Section 7 of the reference manual deals with modules. Section 8 summarizes all the uses of names and the scope rules. Section 9 gives several modules used to define abstract data types.

This completes the language summary; for more details and greater precision consult the reference manual. The rest of this handout consists of three extended examples of specifications and implementations written in Spec: topological sort, editor buffers, and a simple window system.

\section{Example: Topological sort}

Suppose we have a directed graph whose $n+1$ vertexes are labeled by the integers $0 \ldots n$, represented in the standard way by a relation $g ; g(v 1, v 2)$ is true if $v 2$ is a successor of $v 1$, that is, if there is an edge from $v 1$ to v2. We want a topological sort of the vertexes, that is, a sequence that is a permutation of $0 \ldots \mathrm{n}$ in which $\mathrm{v} 2$ follows $\mathrm{v} 1$ whenever $\mathrm{v} 2$ is a successor of v1. Of course this possible only if the graph is acyclic.

MODULE TopologicalSort EXPORT V, G, Q, TopSort $=$

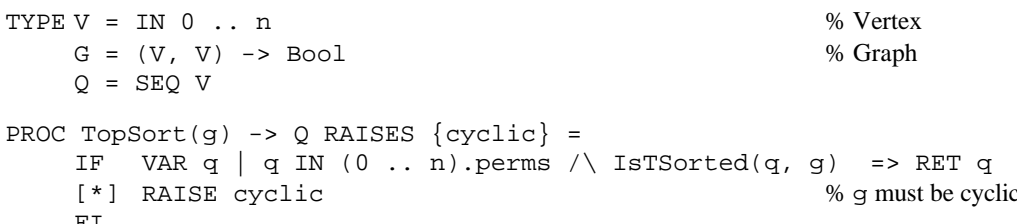

Note that this solution checks for a cyclic graph. It allows any topologically sorted result that is a permutation of the vertexes, because the VAR $q$ in Topsort allows any $q$ that satisfies the two conditions. The perms method on sets and sequences is defined in section 9 of the reference manual; the dom method gives the domain of a function. Topsort is a procedure, not a function, because its result is non-deterministic; we discussed this point earlier when studying SquareRoot. Like that one, this spec has no internal state, since the module has no VAR. It doesn't need one, because it does all its work on the input argument.

The following implementation is from Cormen, Leiserson, and Rivest. It adds vertexes to the front of the output sequence as depth-first search returns from visiting them. Thus, a child is added before its parents and therefore appears after them in the result. Unvisited vertexes are white, nodes being visited are grey, and fully visited nodes are black. Note that all the descendants of a black node must be black. The grey state is used to detect cycles: visiting a grey node means that there is a cycle containing that node. 
This module has state, but you can see that it's just for convenience in programming, since it is reset each time Topsort is called.

MODULE TopSortImpl EXPORT V, G, $\mathrm{Q}$, TopSort $=$

TYPE Color $=$ ENUM $[$ white, grey, black

VAR out : Q

$$
\text { color: V } \rightarrow \text { Color }
$$

$\%$ implements Topsort

$\%$ plus the spec's types

$\%$ every vertex starts white

PROC TopSort $(g) \rightarrow Q$ RAISES $\{$ cyclic $\}=\operatorname{VAR} i:=0$ out $:=\{\}$; color $:=\{* \rightarrow$ white $\}$

DO VAR v $\mid \operatorname{color}(\mathrm{v})=$ white $=>\operatorname{Visit}(\mathrm{v}, \mathrm{g})$ OD; \% visit every unvisited vertex RET out

PROC Visit $(v, g)$ RAISES $\{$ cyclic $\}=$

color $(\mathrm{v}):=$ grey;

DO VAR $v^{\prime} \mid g\left(v, v^{\prime}\right)$ 八 color $\left(v^{\prime}\right) \#$ black $=>$

IF $\operatorname{color}\left(v^{\prime}\right)=$ white $=>\operatorname{Visit}\left(v^{\prime}, g\right)$

[*] RAISE cyclic

OD;

Color $(\mathrm{v}):=$ black: out $:=\{\mathrm{v}\}+$ out

\section{$\%$ pick an successor not done}

$\%$ grey — partly visited

$\%$ add $v$ to front of out

The implementation is as non-deterministic as the spec: depending on the order in which Topsort chooses $v$ and visit chooses $v^{\prime}$, any topologically sorted sequence can result. We could get a deterministic implementation in many ways, for example by taking the smallest node in each case (the min method on sets is defined in section 9 of the reference manual):
VAR $\mathrm{v}:=\{\mathrm{v} 0 \mid \operatorname{color}(\mathrm{v} 0)=$ white $\}$ min
$\operatorname{VAR} \mathrm{v}^{\prime}:=\{\mathrm{v} 0 \mid \mathrm{g}(\mathrm{v}, \mathrm{v} 0)$ in Topsort
VAR $v^{\prime}:=\left\{\mathrm{v} 0 \mid \mathrm{g}(\mathrm{v}, \mathrm{v} 0)\right.$ 八 color $\left(\mathrm{v}^{\prime}\right) \#$ black $\}$.min visit

An implementation in $\mathrm{C}$ would do something like this; the details would depend on the representation of $\mathrm{G}$.

\section{Example: Editor buffers}

A text editor usually has a buffer abstraction. A buffer is a mutable sequence of c's. To get started, suppose that $\mathrm{C}=$ Char and a buffer has two operations,

\section{Get (i) to get character}

Replace to replace a subsequence of the buffer by a subsequence of an argument of type SEQ $c$, where the subsequences are defined by starting position and size.

We can make this specification precise as a Spec class.
CLASS Buffer EXPORT B, C, X, Get, Replace =

$$
\begin{aligned}
\text { TYPE X } & =\text { Nat } \\
C & =\text { Char } \\
\mathrm{B} & =\mathrm{SEQ} C
\end{aligned}
$$

$\%$ indeX in buffer

$\%$ Buffer contents

$\%$ Note: initially empty

$\%$ Note: defined iff $0<=x<b$. size
FUNC Get $(x)->C=R E T b(x)$

' $:$ X) $=$

PROC Replace (from: $X$, size: $X, b$
$\%$ Note: fails if it touches C's that aren't there.

AR b1, b2, b3 $\mid b=b 1+b 2+b 3 八 b 1$.size = from $/ \mathrm{b} 2$.size = size $=>$ $b:=b 1+b^{\prime} \cdot \operatorname{seg}($ from', size') $+b 3$

END Buffer

We can implement a buffer as a sorted array of pieces called a 'piece table'. Each piece contains a SEQ C, and the whole buffer is the concatenation of all the pieces We use binary search to fin a piece, so the cost of Get is at most logarithmic in the number of pieces. Replace may require inserting a piece in the piece table, so its cost is at most linear in the number of pieces. ${ }^{7}$ In particular, neither depends on the number of C's. Also, each Replace increases the size of the array of pieces by at most two.

A piece is a $B$ (in $C$ it would be a pointer to a $B$ ) together with the sum of the length of all the previous pieces, that is, the index in Buffer.b of the first $C$ that it represents; the index is there so that the binary search can work. There are internal routines Locate $(x)$, which uses binary search to find the piece containing $\mathrm{x}$, and Split $(\mathrm{x})$, which returns the index of a piece that starts at $x$, if necessary creating it by splitting an existing piece. Replace calls Split twice to isolate the substring being removed, and then replaces it with a single piece. The time for Replace is linear in pt. size because on the average half of pt is moved when split or Replace inserts a piece, and in half of pt,p.x is adjusted if size' \# size.

7 By using a tree of pieces rather than an array, we could make the cost of Replace logarithmic as well, but to keep things simple we won't do that. See FSImpl in handout 7 for more on this point. 
CLASS BufImpl EXPORT B, C,X, Get, Replace =

\section{$\%$ implements Buffer}

TYPE

$$
\begin{aligned}
& \mathrm{N}=\mathrm{X} \\
& \mathrm{P}=[\mathrm{b}, \mathrm{x}] \\
& \mathrm{PT}=\operatorname{SEQ} \mathrm{P}
\end{aligned}
$$

$\%$ Types as in Buffer, plus $\%$ iNdex in piece table

$\%$ Piece: $\mathrm{x}$ is pos in Buffer.b $\%$ Piece Table

VAR pt $:=$ PT \{\}

ABSTRACTION FUNCTION buffer. $\mathrm{b}=+:\{\mathrm{p}:$ IN pt || $\mathrm{p} . \mathrm{b}\}$

$\%$ buffer.b is the concatenation of the contents of the pieces in $\mathrm{pt}$

INVARIANT (ALL $\mathrm{n}:$ IN pt.dom $\mid \begin{aligned} & \mathrm{pt}(\mathrm{n}) . \mathrm{b} \#\{\} \\ & \wedge \mathrm{pt}(\mathrm{n}) \cdot \mathrm{x}=+\end{aligned}$

$\%$ no pieces are empty, and $x$ is the position of the piece in Buffer. $b$, as promised.

FUNC Get $(\mathrm{x}) \rightarrow \mathrm{C}=$ VAR $\mathrm{p}:=$ pt $(\operatorname{Locate}(\mathrm{x})) \mid \operatorname{RET} \mathrm{p} \cdot \mathrm{b}(\mathrm{x}-\mathrm{p} \cdot \mathrm{x})$

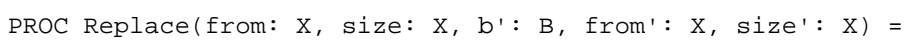

VAR n1 := Split (from); n2 := Split (from + size),

new $:=P\left\{b:=b^{\prime} \cdot \operatorname{seg}(\right.$ from', size'), $x:=$ from $\}$

pt $:=$ pt.sub $(0, \mathrm{n} 1-1)$

+ NonNull (new)

+ pt.sub(n2, pt.size - 1) *AdjustX(size' - size)

PROC Split $(x) \rightarrow \mathrm{N}=$

$\%$ Make pt $(n)$ start at $x$, so pt $(\operatorname{Split}(x)) \cdot x=x$. Fails if $x>b$. size.

$\%$ If pt=abcd|efg|hi, then Split (4) is RET 1 and Split (5) is pt:=abcd|e|fg|hi; RET 2 IF $\mathrm{pt}=\{\} 八 \mathrm{x}=0 \Rightarrow \operatorname{RET} 0$

[*] VAR $n:=\operatorname{Locate}(x), p:=p t(n), b 1, b 2$

$\mathrm{p} \cdot \mathrm{b}=\mathrm{b} 1+\mathrm{b} 2 \Lambda \mathrm{p} \cdot \mathrm{x}+\mathrm{b} 1 \cdot \mathrm{size}=\mathrm{x}=>$

VAR frag1 $:=p\{b:=b 1\}$, frag $2:=p\{b:=b 2, x:=x\}$

pt $:=\operatorname{pt} . \operatorname{sub}(0, \mathrm{n}-1)$

+ NonNull (frag 1$)+$ NonNull (frag 2$)$

+ pt.sub $(\mathrm{n}+1$, pt.size -1$) ;$

FI

RET $\left(\mathrm{b} 1=\{\} \Rightarrow \mathrm{n}\left[{ }^{*}\right] \mathrm{n}+1\right)$

FUNC Locate $(\mathrm{x}) \rightarrow \mathrm{N}=\operatorname{VAR} \mathrm{n} 1:=0, \mathrm{n} 2:=\mathrm{pt}$.size $-1 \mid$

$\%$ Use binary search to find the piece containing $\mathrm{x}$. Yields 0 if $\mathrm{pt}=\{\}$,

$\%$ pt.size-1 if pt\#\{\} $\backslash \mathrm{x}>=\mathrm{b}$. size; never fails. The loop invariant is

$\% \mathrm{pt}=\{\} \backslash / \mathrm{n} 2>=\mathrm{n} 1 /$ pt $(\mathrm{n} 1) \cdot \mathrm{x}\langle=\mathrm{x} /(\mathrm{x}<\mathrm{pt}(\mathrm{n} 2) \cdot \mathrm{x} \backslash / \mathrm{x}>=\mathrm{pt} \cdot$ last.x $)$

\% The loop terminates because $\mathrm{n} 2-\mathrm{n} 1>1=\mathrm{n} 1<\mathrm{n}<\mathrm{n} 2$, so $\mathrm{n} 2-\mathrm{n} 1$ decreases.

DO $\mathrm{n} 2-\mathrm{n} 1>1 \Rightarrow$

VAR $\mathrm{n}:=(\mathrm{n} 1+\mathrm{n} 2) / 2 \mid \operatorname{IF} \mathrm{pt}(\mathrm{n}) \cdot \mathrm{x}<=\mathrm{x}=>\mathrm{n} 1:=\mathrm{n}\left[{ }^{*}\right] \mathrm{n} 2:=\mathrm{n} F I$

$\mathrm{OD} ; \operatorname{RET}\left(\mathrm{x}<\mathrm{pt}(\mathrm{n} 2) \cdot \mathrm{x}=\mathrm{n} 1\left[{ }^{*}\right] \mathrm{n} 2\right)$

FUNC NonNull (p) $\rightarrow$ PT $=\operatorname{RET}(p \cdot b \#\{\} \Rightarrow \operatorname{PT}\{\mathrm{p}\}[*]\{\})$

FUNC AdjustX(dx: Int $) \rightarrow(P \rightarrow P)=\operatorname{RET}(\backslash p \mid p\{x+:=d x\})$

END BufImpl

If subsequences were represented by their starting and ending positions, there would be lots of extreme cases to worry about.
Suppose we now want each $\mathrm{c}$ in the buffer to have not only a character code but also some additional properties, for instance the font, size, underlining, etc. Get and Replace remain the same. In addition, we need a third exported methodApply that applies to each character in a subsequence of the buffer a map function $c \rightarrow c$. Such a function might make all the c's italic, for example, or increase the font size by $10 \%$.

PROC Apply (map: C->C, from: X, size: X) $=$
b := b.sub $(0$, from-1)

$+b \cdot \operatorname{seg}($ from, size) * map

+ b.sub (from + size, b.size-1)

Here is an implementation for Apply that takes time linear in the number of pieces. It works by changing the representation to add a map function to each piece, and in Apply composing the map argument with the map of each affected piece. We need a new version of Get that applies the proper map function, to go with the new representation.

TYPE $\mathrm{P}=[\mathrm{b}, \mathrm{x}, \operatorname{map}: \mathrm{C}->\mathrm{C}]$

$\% \mathrm{x}$ is pos in Buffer.b

ABSTRACTION FUNCTION buffer.b $=+:\{p:$ IN pt ||$p \cdot b * p \cdot m a p\}$

$\%$ buffer.b is the concatenation of the pieces in $p$ with their map's applied.

$\%$ This is the same AF we had before, except for the addition of * $\mathrm{p}$. map.

FUNC Get $(\mathrm{x}) \rightarrow \mathrm{C}=\operatorname{VAR} \mathrm{p}:=\operatorname{pt}(\operatorname{Locate}(\mathrm{x})) \mid \operatorname{RET}$ p.map $(\mathrm{p} \cdot \mathrm{b}(\mathrm{x}-\mathrm{p} \cdot \mathrm{x}))$

PROC Apply (map: C->C, from: X, size: $X)=$

VAR n1 $:=$ Split (from), n2 $:=$ Split (from + size)

pt $:=$ pt.sub $(0, \mathrm{n} 1-1)$

+ pt.sub(n1, n2 - 1$) *(\backslash p \mid p\{m a p:=p \cdot m a p * \operatorname{map}\})$

Note that we wrote Split so that it keeps the same map in both parts of a split piece. We also need to add map := $(\backslash \mathrm{C} \mid \mathrm{C})$ to the constructor for new in Replace.

This implementation was used in the Bravo editor for the Alto, the first what-you-see-is-whatyou-get editor. It is still used in Microsoft Word.

\section{Example: Windows}

A window (the kind on your computer screen, not the kind in your house) is a map from points to colors. There can be lots of windows on the screen; they are ordered, and closer ones block the view of more distant ones. Each window has its own coordinate system; when they are arranged on the screen, an offset says where each window's origin falls in screen coordinates.

MODULE Window EXPORT Get, Paint $=$

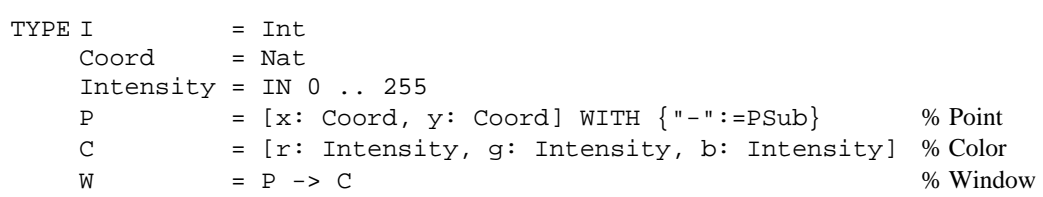

FUNC PSub $(p 1, p 2) \rightarrow P=\operatorname{RET} P\{x:=p 1 \cdot x-p 2 \cdot x, y:=p 1 \cdot y-p 2 \cdot y\}$ 
The shape of the window is determined by the points where it is defined; obviously it need not be rectangular in this very general system. We have given a point a "_" method that computes the vector distance between two points.

A 'window system' consists of a sequence of [w, offset: $P]$ pairs; we call a pair a $v$. The sequence defines the ordering of the windows (closer windows come first in the sequence); it is indexed by 'window number' wn. The offset gives the screen coordinate of the window's

$(0,0)$ point, which we think of as its upper left corner. There are two main operations:

Paint ( wn, $p, c)$ to set the value of $P$ in window wn, and Get $(p)$ to read the value of $p$ in the topmost window where it is defined (that is, the first one in the sequence). The idea is that what you see (the result of Get) is the result of painting the windows from last to first, offsetting each one by its off set component and using the color that is painted later to completely overwrite one painted earlier. Of course real window systems have other operations to change the shape of windows, add, delete, and move them, change their order, and so forth, as well as ways for the window system to suggest that newly exposed parts of windows be repainted, but we won't consider any of these complications.

First we give the spec for a window system initialized with $\mathrm{n}$ empty windows. It is customary to call the coordinate system used by Get the screen coordinates. The $v$. off set field gives the screen coordinate that corresponds to $\{0,0\}$ in v.w. The v.c (p) method below gives the value of v's window at the point corresponding to $p$ after adjusting by v's offset. The state ws is just the sequence of v's. For simplicity we initialize them all with the same offset $\{10,5\}$, which is not too realistic.

Get finds the smallest wn that is defined at $\mathrm{p}$ and uses that window's color at $\mathrm{p}$. This corresponds to painting the windows from last (biggest wN) to first with opaque paint, which is what we wanted. Paint uses window rather than screen coordinates.

The state (the VAR) is a single sequence of windows.

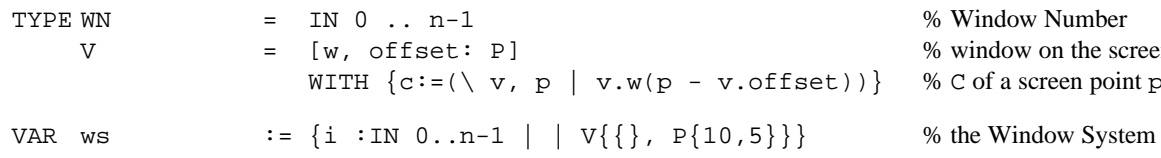

FUNC Get $(\mathrm{p}) \rightarrow C=$ VAR wn $:=\left\{w^{\prime} \mid\right.$ V.c! (ws (wn'), p) $\} \cdot \min \mid \operatorname{RET}$ ws (wn).c(p)

PROC Paint (wn, $p, c)=$ ws $(w n) \cdot w(p):=c$

END Window

Now we give an implementation that only keeps track of the visible color of each point (that is, it just keeps the pixels on the screen, not all the pixels in windows that are covered up by other windows). We only keep enough state to handle Get and Paint.

The state is one w that represents the screen, plus an exposed variable that keeps track of which window is exposed at each point, and the offsets of the windows. This is sufficient to implement Get and Paint; to deal with erasing points from windows we would need to keep more information about what other windows are defined at each point, so that exposed would have a type P $\rightarrow$ SET WN. Alternatively, we could keep track for each window of where it is defined.

Real window systems usually do this, and represent exposed as a set of visible regions of the various windows. They also usually have a 'background' window that covers the whole screen, so that every point on the screen has some color defined; we have omitted this detail from the spec and the implementation.

We need a history variable wH that contains the w part of all the windows. The abstraction function just combines $\mathrm{wH}$ and offset to make ws. The important properties of the implementation are contained in the invariant, from which it's clear that Get returns the answer specified by window. Get. Another way to do it is to have a history variable wsH that is equal to ws. This makes the abstraction function very simple, but then we need an invariant that says offset $(\mathrm{wn})=\mathrm{wsH}(\mathrm{n})$. offset. This is perfectly correct, but it's usually better to put as little stuff in history variables as possible.

MODULE WinImpl EXPORT Get, Paint $=$

$$
\begin{aligned}
& \begin{array}{lll}
\mathrm{W} & :=\mathrm{W}\{\} \\
\text { exposed }: & \mathrm{P} \rightarrow \mathrm{WN}:=\{\}
\end{array} \\
& \text { offset }:=\{i: \text { IN } 0 \ldots n-1|| P(5,10)\} \\
& \mathrm{wH} \quad:=\{i: I N 0 \ldots \mathrm{n}-1|| \mathrm{W}\{\}\}
\end{aligned}
$$

$\%$ no points defined

$\%$ which wn shows at $\mathrm{p}$

$\%$ history variable

ABSTRACTION FUNCTION wS $=(\backslash \mathrm{wn} \mid \mathrm{V}\{\mathrm{w}:=\mathrm{wH}(\mathrm{wn})$, offset $:=\operatorname{offset}(\mathrm{wn})\})$ INVARIANT

$$
\begin{aligned}
& \text { (ALL } \mathrm{p} \mid \mathrm{w} ! \mathrm{p}=\text { exposed! } \mathrm{p} \\
& \bigwedge(w ! p=\Rightarrow \quad\{w n \mid V \cdot c !(w s(w n), p)\} \cdot \min =\operatorname{exposed}(p) \\
& 八 \mathrm{w}(\mathrm{p})=\mathrm{ws}(\operatorname{exposed}(\mathrm{p})) \cdot \mathrm{c}(\mathrm{p}))
\end{aligned}
$$

The invariant says that each visible point comes from some window, exposed tells the topmost window that defines it, and its color is the color of the point in that window. Note that for convenience the invariant uses the abstraction function; of course we could have avoided this by expanding it in line, but there is no reason to do so, since the abstraction function is a perfectly good function.

FUNC Get $(p) \rightarrow C=\operatorname{RET} w(p)$

PROC Paint (wn, $p, c)=$

$$
\begin{aligned}
& \text { VAR } \mathrm{pO} \mid \mathrm{p}=\mathrm{p} 0-\operatorname{offset}(\mathrm{wn}) \Rightarrow \\
& \operatorname{IF} \mathrm{wn}<=\text { exposed }(\mathrm{p} 0) \Rightarrow \mathrm{w}(\mathrm{p} 0):=\mathrm{c} \text {; exposed }(\mathrm{p} 0) \begin{array}{l}
\% \text { theen coordinate } \\
:=\mathrm{wn}\left[{ }^{*}\right] \text { SKIP FI } \\
\% \text { update the history var }
\end{array} \\
& \mathrm{wH}(\mathrm{wn})(\mathrm{p}):=\mathrm{C}
\end{aligned}
$$

END WinImpl 


\section{Index}

!, 9

(...),

$;, 14$

[*], 13

\{* $\rightarrow\}, 8$

$<\ldots>>, 3$

$==>, 3,10$

$=>, 3,12$

$\rightarrow, 4,10$

algorithm, 5

ALL, 3, 10
APROC, 4, 7

arbitrary relation, 15

array, 8

assignment, 3, 8, 12

atomic, 16

atomic actions, 3

atomic command, 6

atomic procedure, 7

BEGIN, 14

behavior, 2
Bool, 8

choice, 12

choose, $4,10,15$

class, 19

combination, 10

command, 3, 6, 12

communicate, 2

compose, 11

composition, 16

conditional, 11,
constant, 7

constructor, 8

contract, 2

declare, 7

defined, 9

Dijkstra, 1

DO, 4,16

else, 14

END, 14

EXCEPT, 14

exception, 5

exceptional outcome, 6

existential quantifier, 5,10

expression, 4,6

FI, 14

function, 7, 8, 10

function constructor, 8,10

function declaration, 11

functional behavior, 2

global, 17

guard, 3, 12

handler, 5

hierarchy, 17

history, 2, 6

if, 3,12

implementer,

implementer, 2

infinite, 3

Int, 8

invocation, 12

lambda expression, 8

local, 3, 17

loop, 16

meaning

of an atomic command, 6

of an expression, 6

method, 7, 11, 16

module, 7,17

name, 6

name space, 17

Nelson, 1

non-atomic command, 6

non-atomic semantics, 6

non-deterministic, 4, 5, 6, 13, 15

normal outcome, 6,14

OD, 4,16

operator, 12

organizing your program, 7

outcome, 6

parameterized, 17

precedence, 14,15

precisely, 2

predicate, $3,10,12$

PROC, 7

procedure, 7

program, 2, 4, 7

program counter, 6

puantifier $3,4,10$

quatife, 3,4

RAISES, 5

record constructor, 8 relation, 6

repetition, 16

RET, 4

routine, 7

seq, 11

$\mathrm{SEQ}, 3$

sequence, 8,16

sequential program, 6

set, 3,8

set constructor, 9

set of sequences of states, 6

side 7

side-ect,

pec, 2

pecification, 2,4

state, 2, 6

state transition,

state variable, 6

strongly typed, 8

such that, 3

SUCHTHAT, 8

erminates, 16

then, 3,12

hread, 7

THREAD, 7

transition, 2, 6

wo-level hierarchy, 7

type, 7

undefined, 8, 12

universal quantifier, 3,10

value, 6

VAR, $3,4,15$

variable, 6,7

variable introduction, 15

WITH, 11
This page intentionally left blank 


\section{Operators $(\S 5, \S 9)$ \\ Op Pr Type $\mathrm{x}$ op $\mathrm{y}$ is \\ - 9 Any x's y field/method \\ IS 8 Any does $x$ have type $y$ ? \\ AS 8 Any $x$ with type $y$ \\ ** 8 Int $\mathrm{x}^{\mathrm{y}}$ \\ * 7 Int $\mathrm{x} \times \mathrm{y}$ \\ func composition \\ relation composition \\ / 7 Int $\mathrm{x} / \mathrm{y}$ rounded to 0 \\ // 7 Int mod: $\mathrm{x}-(\mathrm{x} / \mathrm{y})^{\star} \mathrm{y}$ \\ +6 Int $x+y$ \\ func overlay \\ seq concatenation \\ - 6 Int $x-y$ \\ set set difference \\ seq multiset diff \\ ! 6 func $x$ defined at $y$ \\ !! 6 func $\mathrm{x}$ ! $\mathrm{y} \wedge \mathrm{x}(\mathrm{y})$ not ex \\ .. 5 Int seq $\{x, x+1, \ldots, y\}$ \\ $=4$ Any $x=y$ \\ \# 4 Any $x \neq y$ \\ $=4$ seq $\mathrm{x}=\mathrm{y}$ as multisets \\ $<=4$ Int $x \leq y$ \\ set $\quad \mathrm{x} \subseteq \mathrm{y}$ (subset) \\ seq $\quad x$ a prefix of $y$ \\ $<<=4$ seq $x$ a sub-seq of $y$ \\ IN 4 set/seq $x \in y$ (member) \\ $\sim 3$ Bool not $x$ (unary) \\ $\wedge 2$ Bool $\mathrm{x} \wedge \mathrm{y}$ (and) \\ set $x \cap y$ (intersection) \\ $\backslash / 1$ Bool $x \vee y$ (or) set $\quad \mathrm{x} \cup \mathrm{y}$ (union) \\ $==>0$ Bool $x$ implies $y$}

Operators associate to the left.

\section{Expression forms (\$ 5)}

$f(e)$

op : sq

\begin{tabular}{ll|l}
$($ ALL & $x$ & pred $)$ \\
$(\operatorname{EXISTS}$ & $\mathrm{x}$ & pred $)$ \\
$($ pred $=>$ & $e_{1}$ & {$\left[{ }^{*}\right]$}
\end{tabular}

func

set/seq

Bool

Bool

Any

\section{Constructors $(\$ 5)$}

\begin{tabular}{|c|c|c|}
\hline$\left\{e_{1}, \ldots, e_{n}\right\}$ & set & with these members \\
\hline$\{i:$ Nat $|i<3| i * \star 2\}$ & & of $i^{2}$ s where $i<3$ \\
\hline$f\left\{e_{1}->e_{2}\right\}$ & func & $\mathrm{f}$ except $=e_{2}$ at $\arg e_{1}$ \\
\hline$\{\star->$ e $\}$ & & $=e$ at every arg \\
\hline$(\backslash i:$ Int $\mid i<3)$ & & lambda (also LAMBDA) \\
\hline$\left\{e_{1}, \ldots, e_{n}\right\}$ & seq & of e's in this order \\
\hline$\{i:$ IN $0 \ldots 5|| i * * 2\}$ & & $\{0,1,4,9,16,25\}$ \\
\hline$i:=0$ BY $i+1$ WHILE $i<6|| i *$ & & same \\
\hline$\left(e_{1}, \ldots, e_{n}\right)$ & tuple & of e's \\
\hline$\left\{f_{1}:=e_{1}, \ldots, f_{n}:=e_{n}\right\}$ & record & $r$ except $f_{1}=e_{1} \ldots$ \\
\hline
\end{tabular}

\section{Methods (§ 9)}

set

Ops: 八 \/ - <= IN, op:

size number of members

choose some member of $s$

seq $\quad s$ as some sequence

pred $\quad \mathrm{s} \cdot \operatorname{pred}(\mathrm{x})=(\mathrm{x} \in \mathrm{s})$

$\mathrm{fmax} / \mathrm{min}$ some $\max / \mathrm{min}$ by $\mathrm{f}_{1}$

$\max / \mathrm{min}$ some $\max / \mathrm{min}$ by $<=$

set/seq perms set of all perms of $\mathrm{sq}$

fsort sq sorted (q stably) by $f_{1}$

sort sq sorted (q stably) by $<=$

func Ops: * + ! !

dom, rng domain, range

inv inverse

restrict domain to set $s_{1}$

rel $\quad r(x, y)=(f(x)=y)$

predicate set $\quad \mathrm{s}=\{\mathrm{x} \mid \operatorname{pred}(\mathrm{x})\}$

relation Ops: * and func +

dom, rng domain, range

inv inverse

set $\mathrm{F} \quad \mathrm{f}(\mathrm{x})=\{\mathrm{y} \mid \mathrm{r}(\mathrm{x}, \mathrm{y})\}$

func $\quad f(x)=\operatorname{set} F(x)$.choose

graph ispath is $q_{1}$ a path in $g$ ?

closure transitive closure of $\mathrm{g}$

seq Ops:..$+-<=<<=$ IN, op :, func * !

also see size number of elements

func above head $q(0)$

tail $\{q(1), \ldots, q(q$. size-1) $\}$

remh remove head = tail

last q(q.size-1)

reml $\quad\{q(0), \ldots, q(q . s i z e-2)\}$

sub $\quad\left\{q\left(i_{1}\right), \ldots, q\left(i_{2}\right)\right\}$

seg $\left\{q\left(i_{1}\right), \ldots\right\}, i_{2}$ elements

fill $i_{2}$ copies of $x_{1}$

lexLE $\quad q$ lexically $<=q_{1}$ by $f_{2}$ ?

fsorter perm sorts $q$ stably by $f_{1}$

count number of $x_{1}$ 's in $q$

set $\quad$ q as a set, $=q . r n g$

tuple tuple with q's values

uple seq

function invocation

$\mathrm{sq}(0)$ op $\mathrm{sq}(1) \ldots$

$\operatorname{pred}\left(x_{1}\right) \wedge \ldots \wedge \operatorname{pred}\left(x_{n}\right)$

$\operatorname{pred}\left(x_{1}\right) \vee \ldots \vee \operatorname{pred}\left(x_{n}\right)$

$e_{1}$ if pred else $e_{2}$
Types (\$ 4)

Any, Null, Bool, Int, basic

Nat, Char, String

SET T, IN S set

$\mathrm{T}_{1} \rightarrow \mathrm{T}_{2}$

APROC $\mathrm{T}_{1} \rightarrow \mathrm{T}_{2}$

PROC $\quad \mathrm{T}_{1} \rightarrow \mathrm{T}_{2}$

SEQ T

$\left(\mathrm{T}_{1}, \ldots, \mathrm{T}_{\mathrm{n}}\right)$

$\left[f_{1}: T_{1}, \ldots, f_{n}: T_{n}\right]$

$\left(\mathrm{T}_{1}+\ldots+\mathrm{T}_{\mathrm{n}}\right)$

T WITH $\left\{\mathrm{m}_{1}:=\mathrm{f}_{1}, \ldots\right\}$

T SUCHTHAT pred

function

procedures

sequence

tuple

record

union

add methods

limit values

Commands (§ 6) $P r$

SKIP, HAVOC,

simple

RET e, RAISE ex

$\mathrm{p}(\mathrm{e})$

$x:=e, x:=p(e)$,

invocation

$\left(\mathrm{x}_{1}, \ldots\right):=\mathrm{e}$

$\mathrm{C}_{1} \operatorname{EXCEPT}$ ex $=>\mathrm{C}_{2} \quad 3$ handle ex

$\mathrm{C}_{1} ; \mathrm{C}_{2}$

2 sequential

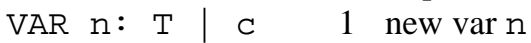

pred $=>\mathrm{C}$

1 if (guarded cmd)

$\mathrm{C}_{1}$ [ ] $\mathrm{C}_{2}$

0 or (ND choice)

$\mathrm{C}_{1}[*] \quad \mathrm{C}_{2}$

$<$ C $>>$

BEGIN C END

0 else

atomic $\mathrm{C}$

brackets

IF C FI

DO $C$ OD

loop until fail

Command operators associate to the left, but EXCEPT associates to the right.

\section{Modules (\$ 7)}

MODULE/CLASS M

$$
\left[\mathrm{T}_{1} \mathrm{WITH}\left\{\mathrm{m}_{1}: \mathrm{T}_{11}->\mathrm{T}_{12}, \ldots\right\}, \ldots\right]
$$

EXPORT $n_{1}, \ldots=$

TYPE $\mathrm{T}_{1}=\mathrm{SET} \mathrm{T}_{2}$

$\mathrm{T}_{3}=\operatorname{ENUM}\left[\mathrm{n}_{1}, \ldots\right]$

CONST $n: \mathrm{T}:=\mathrm{e}$

$\mathrm{VAR} \quad \mathrm{n}: \mathrm{T}:=\mathrm{e}$

EXCEPTION ex $=\left\{\operatorname{ex}_{11}, \ldots\right\}+\mathrm{ex}_{2}+.$.

FUNC $f\left(n_{1}: T_{1}, \ldots\right) \rightarrow T=c$

APROC, PROC, THREAD similarly

END M

Naming conventions (except in 'Operators')

c command op operator

e expression $\mathrm{p}$ proccedure

ex exception $P r$ precedence

$\mathrm{f}$ function, field $\mathrm{q}$ sequence

$g$ graph $r$ record, relation

$i$ Int $s$ set

$m$ method $T$ type

$n$ name $x$ Any

$z_{i} \quad i$ th extra argument of a method, or one of several like non-terminals in a rule

$\S \quad$ a section of the Spec reference manual 


\section{How to Write a Spec}

Figure out what the state is.

Choose the state to make the spec simple and clear, not to match the code.

\section{Describe the actions.}

What they do to the state.

What they return.

\section{Helpful hints}

Notation is important, because it helps you to think about what's going on.

Invent a suitable vocabulary.

Less is more. Less state is better. Fewer actions are better.

More non-determinism is better, because it allows more implementations.

In distributed systems, replace the separate nodes with non-determinism in the spec.

Pass the coffee-stain test: people should want to read the spec.

I'm sorry I wrote you such a long letter; I didn't have time to write a short one. - Pascal

\section{How to Design an Implementation}

\section{Write the spec first.}

\section{Dream up the idea of the implementation.}

Embody the key idea in the abstraction function.

\section{Check that each implementation action simulates some spec actions.}

Add invariants to make this easier. Each action must maintain them.

Change the implementation (or the spec, or the abstraction function) until this works.

\section{Make the implementation correct first, then efficient.}

More efficiency means more complicated invariants.

You might need to change the spec to get an efficient implementation.

Measure first before making anything faster.

An efficient program is an exercise in logical brinkmanship. - Dijkstra 


\section{Spec Reference Manual}

Spec is a language for writing specifications and the first few stages of successive refinement towards a practical implementation. As a specification language it includes constructs (quantifiers, backtracking or non-determinism, some uses of atomic brackets) which are impractical in a final implementation; they are there because they make it easier to write clear, unambiguous and suitably general specifications. If you want to write a practical program, avoid them.

This document defines the syntax of the language precisely and the semantics informally. You should read the Introduction to Spec (handout 3) before trying to read this manual. In fact, this manual is intended mainly for reference; rather than reading it carefully, skim through it, and then use the index to find what you need. For a precise definition of the atomic semantics read Atomic Semantics of Spec. (handout 9). Handout 17 on Formal Concurrency gives the nonatomic semantics semi-formally.

\section{Overview}

Spec is a notation for writing specifications for a discrete system. What do we mean by a specification? It is the allowed sequences of transitions of a state machine. So Spec is a notation for describing sequences of transitions of a state machine.

Expressions and commands

The Spec language has two essential parts:

An expression describes how to compute a value as a function of other values, either constants or the current values of state variables.

A command describes possible transitions, or changes in the values of the state variables.

Both are based on the state, which in Spec is a mapping from names to values. The names are called state variables or simply variables: in the examples below they are $i$ and $j$.

There are two kinds of commands:

An atomic command describes a set of possible transitions. For instance, the command $<<:=i+1>>$ describes the transitions $i=1 \rightarrow i=2, \quad i=2 \rightarrow i=3$, etc. (Actually, many transitions are summarized by $i=1 \rightarrow i=2$, for instance, $(i=1, j=1) \rightarrow(i=2, j=1)$ and $(i=1$, $j=15) \rightarrow(i=2, \quad j=15))$. If a command allows more than one transition from a given state we say it is non-deterministic. For instance, the command, $\langle<i:=1$ [] $i:=i+1\rangle$ allows the transitions $i=2 \rightarrow i=1$ and $i=2 \rightarrow i=3$. More on this in Atomic Semantics of Spec.

A non-atomic command describes a set of sequences of states. More on this in Formal Concurrency.

A sequential program, in which we are only interested in the initial and final states, can be described by an atomic command.
Spec's notation for commands, that is, for changing the state, is derived from Edsger Dijkstra's guarded commands (E. Dijkstra, A Discipline of Programming, Prentice-Hall, 1976) as extended by Greg Nelson (G. Nelson, A generalization of Dijkstra's calculus, ACM TOPLAS 11, 4, Oct. 1989, pp 517-561). The notation for expressions is derived from mathematics.

\section{Organizing a program}

In addition to the expressions and commands that are the core of the language, Spec has four other mechanisms that are useful for organizing your program and making it easier to understand.

A routine is a named computation with parameters (passed by value). There are four kinds:

A function is an abstraction of an expression.

An atomic procedure is an abstraction of an atomic command.

A general procedure is an abstraction of a non-atomic command.

A thread is the way to introduce concurrency.

A type is a stylized assertion about the set of values that a name can assume. A type is also an easy way to group and name a collection of routines, called its methods, that operate on values in that set.

An exception is a way to report an unusual outcome.

A module is a way to structure the name space into a two-level hierarchy. An identifier i declared in a module $m$ is known as $i$ in $m$ and as $m$. $i$ throughout the program. A class is a module that can be instantiated many times to create many objects.

A Spec program is some global declarations of variables, routines, types, and exceptions, plus a set of modules each of which declares some variables, routines, types, and exceptions.

\section{Outline}

This manual describes the language bottom-up:

Lexical rules

Types

Expressions

Commands

Modules

At the end there are two sections with additional information: Scope rules

Built-in methods for set, sequence, and routine types.

There is also an index. The Introduction to Spec has a one-page language summary. 


\section{Grammar rules}

Nonterminal symbols are in lower case; terminal symbols are punctuation other than $::=$, or are quoted, or are in upper case.

Alternative choices for a nonterminal are on separate lines.

symbol* denotes zero of more occurrences of symbol.

The symbol empty denotes the empty string.

If $\mathrm{x}$ is a nonterminal, the nonterminal $\mathrm{xList}$ is defined by

xList

$$
\begin{aligned}
::= & x \\
& x, x \text { List }
\end{aligned}
$$

A comment in the grammar runs from \% to the end of the line; this is just like Spec itself.

A [n] in a comment means that there is an explanation in a note labeled [n] that follows this chunk of grammar.

\section{Lexical rules}

The symbols of the language are literals, identifiers, keywords, operators, and the punctuation ( ) $[$ ] \{\}$, ;:$ : $\mid<>>>=>$ [ ] [*]. Symbols must not have embedded white space. They are always taken to be as long as possible.

A literal is a decimal number such as 3765 , a quoted character such as ' $\mathrm{x}$ ', or a double-quoted string such as "Hello\n".

An identifier (id) is a letter followed by any number of letters, underscores, and digits followed by any number of ' characters. Case is significant in identifiers. By convention type and procedure identifiers begin with a capital letter. An identifier may not be the same as a keyword. The predefined identifiers Any, Bool, Char, Int, Nat, Null, String, true, false, and nil are declared in every program. The meaning of an identifier is established by a declaration; see section 8 on scope for details. Identifiers cannot be redeclared.

By convention keywords are written in upper case, but you can write them in lower case if you like; the same strings with mixed case are not keywords, however. The keywords are

$\begin{array}{llllll}\text { ALL } & \text { APROC } & \text { AS } & \text { BEGIN } & \text { BY } & \text { CLASS } \\ \text { CONST } & \text { DO } & \text { END } & \text { ENUM } & \text { EXCEPT } & \text { EXCEPTION } \\ \text { EXISTS } & \text { EXPORT } & \text { FI } & \text { FUNC } & \text { HAVOC } & \text { IF } \\ \text { IN } & \text { IS } & \text { LAMBDA } & \text { MODULE } & \text { OD } & \text { PROC } \\ \text { RAISE } & \text { RAISES } & \text { RET } & \text { SEQ } & \text { SET } & \text { SKIP } \\ \text { SUCHTHAT } & \text { THREAD } & \text { TYPE } & \text { VAR } & \text { WHILE } & \text { WITH }\end{array}$

An operator is any sequence of the characters !@\# $\$^{\wedge} \AA^{*}-+=:$. $<>? 八 \mid \sim$ except the sequences

: . $\mid<<>>:==>->$ (these are punctuation), or one of the keyword operators AS, IN, and IS.

A comment in a Spec program runs from a outside of quotes to the end of the line. It does not change the meaning of the program.

\section{Types}

A type defines a set of values; we say that a value $\mathrm{v}$ has type $\mathrm{T}$ if $\mathrm{v}$ is in $\mathrm{T}$ 's set. The sets are not disjoint, so a value can belong to more than one set and therefore can have more than one type. In addition to its value set, a type also defines a set of routines (functions or procedures) called its methods; a method normally takes a value of the type as its first argument.

An expression has exactly one type, determined by the rules in section 5; the result of the expression has this type unless it is an exception.

The picky definitions given on the rest of this page are the basis for Spec's type-checking. You can skip them on first reading, or if you don't care about type-checking.

About unions: If the expression e has type $\mathrm{T}$ we say that e has a routine type $\mathrm{w}$ if $\mathrm{T}$ is a routine type $\mathrm{W}$ or if $\mathrm{T}$ is a union type and exactly one type $\mathrm{W}$ in the union is a routine type. Under corresponding conditions we say that e has a sequence or set type, or a record type with a field $f$.

Two types are equal if their definitions are the same (that is, have the same parse trees) after al type names have been replaced by their definitions and all WITH clauses have been discarded. Recursion is allowed; thus the expanded definitions might be infinite. Equal types define the same value set. Ideally the reverse would also be true, but type equality is meant to be decided by a type checker, whereas the set equality is intractable.

A type $\mathrm{T}$ fits a type $\mathrm{U}$ if the type-checker thinks they may have some values in common. This can only happen if they have the same structure, and each part of $\mathrm{T}$ fits the corresponding part of $\mathrm{U}$. 'Fits' is an equivalence relation. Precisely, $T$ fits $U$ if:

$$
\mathrm{T}=\mathrm{U} \text {. }
$$

$\mathrm{T}$ is $\mathrm{T}^{\prime}$ SUChthat $\mathrm{F}$ or $\left(\ldots+\mathrm{T}^{\prime}+\ldots\right)$ and $\mathrm{T}^{\prime}$ fits $\mathrm{U}$, or vice versa. There may be no values in common, but the type-checker can't analyze the sUснтнAт clauses to find out.

$\mathrm{T}$ and $\mathrm{U}$ are tuples of the same length and each component of $\mathrm{T}$ fits the corresponding component of $\mathrm{U}$.

$\mathrm{T}$ and $\mathrm{U}$ are record types, and for every decl $i d: \mathrm{T}$ ' in $\mathrm{T}$ there is a corresponding decl $\mathrm{id}$ : $U^{\prime}$ in $U$ such that $T$ ' fits $U$ ', or vice versa.

T=T1->T2 RAISES EXt and U=U1->U2 RAISES EXu, or one or both RAISES are missing, and T1 fits U1 and T2 fits U2. Similar rules apply for PROC and APROC types.

$T=S E T T^{\prime}$ and $U=S E T U^{\prime}$ and $T^{\prime}$ fits $U^{\prime}$.

$T=$ Int $->T^{\prime}$ or SEQ $T^{\prime}$ and $U=S E Q U^{\prime}$ and $T^{\prime}$ fits $U^{\prime}$.

T includes $\mathrm{U}$ if the same conditions apply with "fits" replaced by "includes", all the "vice versa" clauses dropped, and in the $->$ rule "T1 fits U1" replaced by " $\mathrm{U} 1$ includes $\mathrm{T} 1$ and EXt is a superset of EXu". If T includes U then T's value set includes U's value set; again, the reverse is intractable.

An expression e fits a type $U$ in state $s$ if e's type fits $U$ and the result of e in state $s$ has type $U$ or is an exception; in general this can only be checked at runtime unless $u$ includes e's type. The check that e fits $\mathrm{T}$ is required for assignment and routine invocation; together with a few other checks it is called type-checking. The rules for type-checking are given in sections 5 and 6. 
type $\begin{aligned}::= & \text { name } \\ & \text { "Any" } \\ & \text { "Null" } \\ & \text { "Char" } \\ & \text { "String" } \\ & \text { "Int" } \\ & \text { "Nat" } \\ & \text { SEQ type } \\ & \text { SET type } \\ & \text { ( typeList } \\ & \text { [ declLis } \\ & \text { ( union) } \\ & \text { aType -> } \\ & \text { APROC aTy } \\ & \text { PROC aTy } \\ & \text { type wITH } \\ & \text { type SUCH } \\ & \text { IN exp } \\ & \text { id [ type } \\ ::= & \text { id . id } \\ & \text { id } \\ & \text { type . id } \\ := & \text { id }: \text { type }\end{aligned}$

id

$::=$ type + type

union + type

aType $\quad::=()$

returns $\quad::=$ empty

$\rightarrow \rightarrow$ type

RAISES exceptionset

exceptionset $::=\{$ exceptionList $\}$

name

exceptionset $\checkmark /$

exceptionset $\backslash$ / exceptionset $\%$ set union
exceptionset - exceptionset \% set differen

exception $\quad::=$ id

$::=$ id

stringLiteral

methodDef $\quad::=$ method $:=$ name

$\%$ name of a type

$\%$ every value has this type

$\%$ with value set $\{$ nil $\}$

$\%$ with value set $\{$ true, false

$\%$ like an enumeration

$\%=\mathrm{SEQ}$ Char

$\%$ integers

\% naturals: non-negative integers

$\%$ sequence [1]

$\%$ set

$\%$ tuple; ( $\mathrm{T}$ ) is the same as $\mathrm{T}$

$\%$ record with declared fields

$\%$ union of the types

$\%$ function [2]

$\%$ atomic procedure

$\%$ non-atomic procedure

$\%$ attach methods to a type [3]

$\%$ restrict the value set [4]

$\%=\mathrm{T}$ SUCHTHAT $(\backslash \mathrm{t}: \mathrm{T} \mid \mathrm{t}$ IN exp

$\%$ where exp's type has an IN method

$\%$ type from a module [5]

$\%$ the first id denotes a module

$\%$ short for $\mathrm{m}$. id if id is declared

$\%$ in the current module $\mathrm{m}$, and for

$\%$ Global.id if id is declared globally

$\%$ the id method of type

$\%$ id has this type

$\%$ short for id: Id [6]

$\%$ only for procedures

$\%$ the exceptions it can return

\section{$\%$ a set of exceptions}

$\%$ declared as an exception set

$\%$ means "id"

method

$\%$ the string must be an operator
$\%$ other than "=" or "\#" (see section 3)

$\%$ name is a routine
The ambiguity of the type grammar is resolved by taking $->$ to be right associative and giving WITH and RAISES higher precedence than $\rightarrow$.

[1] A SEQ $\mathrm{T}$ is just a function from $\{0,1, \ldots$, size-1 $\}$ to $T$. That is, it is short for (Int->T) SUCHTHAT $(\backslash \mathrm{f}$ : Int->T $\mid$ (EXISTS size: Int | WITH $\{$ see section 9$\}$.

(ALL $i$ : Int $\mid f ! i=(i$ IN $0 \ldots$ size-1)) )

This means that invocation, !, and * work for a sequence just as they do for any function. In addition, there are many other useful operators on sequences; see section 9. The string type is just SEQ Char; there are String literals, defined in section 5.

[2] A $T->U$ value is a partial function from a state and a value of type $T$ to a value of type $U$. A $T->U$ RAISES xs value is the same except that the function may raise the exceptions in $x s$.

[3] We say $m$ is a method of $\mathrm{T}$ defined by $\mathrm{f}$, and denote $\mathrm{f}$ by $\mathrm{T} . \mathrm{m}$, if

$\mathrm{T}=\mathrm{T}^{\prime} \operatorname{WITH}\{\ldots, \mathrm{m}:=\mathrm{f}, \ldots\}$ and $\mathrm{m}$ is an identifier or is "op" where op is an operator (the construct in braces is a methodDefList), or

$T=T^{\prime}$ WITH $\{$ methodDefList $\}, m$ is not defined in methodDefList, and $m$ is a method of $T$ ' defined by $f$, or

$T=\left(\ldots+T^{\prime}+\ldots\right), m$ is a method of $T^{\prime}$ defined by $f$, and there is no other type in the union with a method $\mathrm{m}$.

There are two special forms for invoking methods: e1 infixop e2 or prefixop e, and e1.id (e2) or e.id or e.id(). They are explained in notes [1] and [3] to the expression grammar in the next section. This notation may be familiar from object-oriented languages. Unlike many such languages, Spec makes no provision for varying the method in each object, though it does allow inheritance and overriding.

A method doesn't have to be a routine, though the special forms won't type-check unless the method is a routine. Any method $m$ of $\mathrm{T}$ can be referred to by T.m.

[4] In $T$ SUchthat $f, f$ is a predicate on $T$ 's, that is, a function ( $T \rightarrow$ Bool). The type T SUCHтHAT $f$ has the same methods as $T$, and its value set is the values of $T$ for which $f$ is true. See section 5 for primary.

[5] If a type is defined by $m$ [typeList] . id and $m$ is a parameterized module, the meaning is $\mathrm{m}^{\prime}$. id where $\mathrm{m}^{\prime}$ is defined by MODULe $\mathrm{m}^{\prime}=\mathrm{m}$ [typelist] END $\mathrm{m}^{\prime}$. See section 7 for a full discussion of this kind of type.

[6] Id is the id of a type, obtained from id by dropping trailing ' characters and digits, and capitalizing the first letter or all the letters (it's an error if these capitalizations yield different identifiers that are both known at this point). 


\section{Expressions}

An expression is a partial function from states to results; results are values or exceptions. That is, an expression computes a result for a given state. The state is a function from names to values. This state is supplied by the command containing the expression in a way explained later. The meaning of an expression (that is, the function it denotes) is defined informally in this section. The meanings of invocations and lambda function constructors are somewhat tricky, and the informal explanation here is supplemented by a formal account in Atomic Semantics of Spec Because expressions don't have side effects, the order of evaluation of operands is irrelevant (but see [5] and [13]).

Every expression has a type. The result of the expression is a member of this type if it is not an exception. This property is guaranteed by the type-checking rules, which require an expression used as an argument, the right hand side of an assignment, or a routine result to fit the type of the formal, left hand side, or routine range (see section 4 for the definition of 'fit'). In addition, expressions appearing in certain contexts must have suitable types: in e1 (e2), e1 must have a routine type; in $\mathrm{e} 1+\mathrm{e} 2$, e1 must have a type with a "+" method, etc. These rules are given in detail in the rest of this section. A union type is suitable if exactly one of the members is suitable. Also, if $\mathrm{T}$ is suitable in some context, so are $\mathrm{T}$ WITH $\{\ldots\}$ and $\mathrm{T}$ SUCHTHAT $\mathrm{f}$

An expression can be a literal, a variable known in the scope that contains the expression, or a function invocation. The form of an expression determines both its type and its result in a state:

literal has the type and value of the literal.

name has the declared type of name and its value in the current state, state ("name"). The form T.m (where T denotes a type) is also a name; it denotes the m method of T. Note that if name is $i d$ and $i d$ is declared in the current module $m$, then it is short for $m . i d$.

invocation $f(e)$ : $f$ must have a function (not procedure) type U $>$ T RAISES EX or U->T (note that a sequence is a function), and e must fit $U$; then $f(e)$ has type T. In more detail, if $f$ has result $r f$ and e has type $U$ ' and result re, then $U$ ' must fit $U$ (checked statically) and re must have type $U$ (checked dynamically if $U$ ' involves a union or SUснтнAт; if the dynamic check fails the result is a fatal error). Then $f(e)$ has type $T$.

If either $r f$ or $r e$ is undefined, so is $f(e)$. Otherwise, if either is an exception, that exception is the result of $f(e)$; if both are, $r f$ is the result.

If both $r f$ and $r e$ are normal, the result of $r f$ at $r e$ can be:

A normal value, which becomes the result of $f(e)$.

An exception, which becomes the result of $f(e)$. If $\mathrm{r} f$ is defined by a function body that loops, the result is a special looping exception that you cannot handle.

Undefined, in which case $f(e)$ is undefined and the command containing it fails (has no outcome) - failure is explained in section 6 .

A function invocation in an expression never affects the state. If the result is an exception, the containing command has an exceptional outcome; for details see section 6 .

The other forms of expressions (e.id, constructors, prefix and infix operators, combinations, and quantifications) are all syntactic sugar for function invocations, and their results are obtained by the rule used for invocations. There is a small exception for conditionals [5] and for the conditional logical operators $\wedge, \backslash /$, and $==>$ that are defined in terms of conditionals [13].

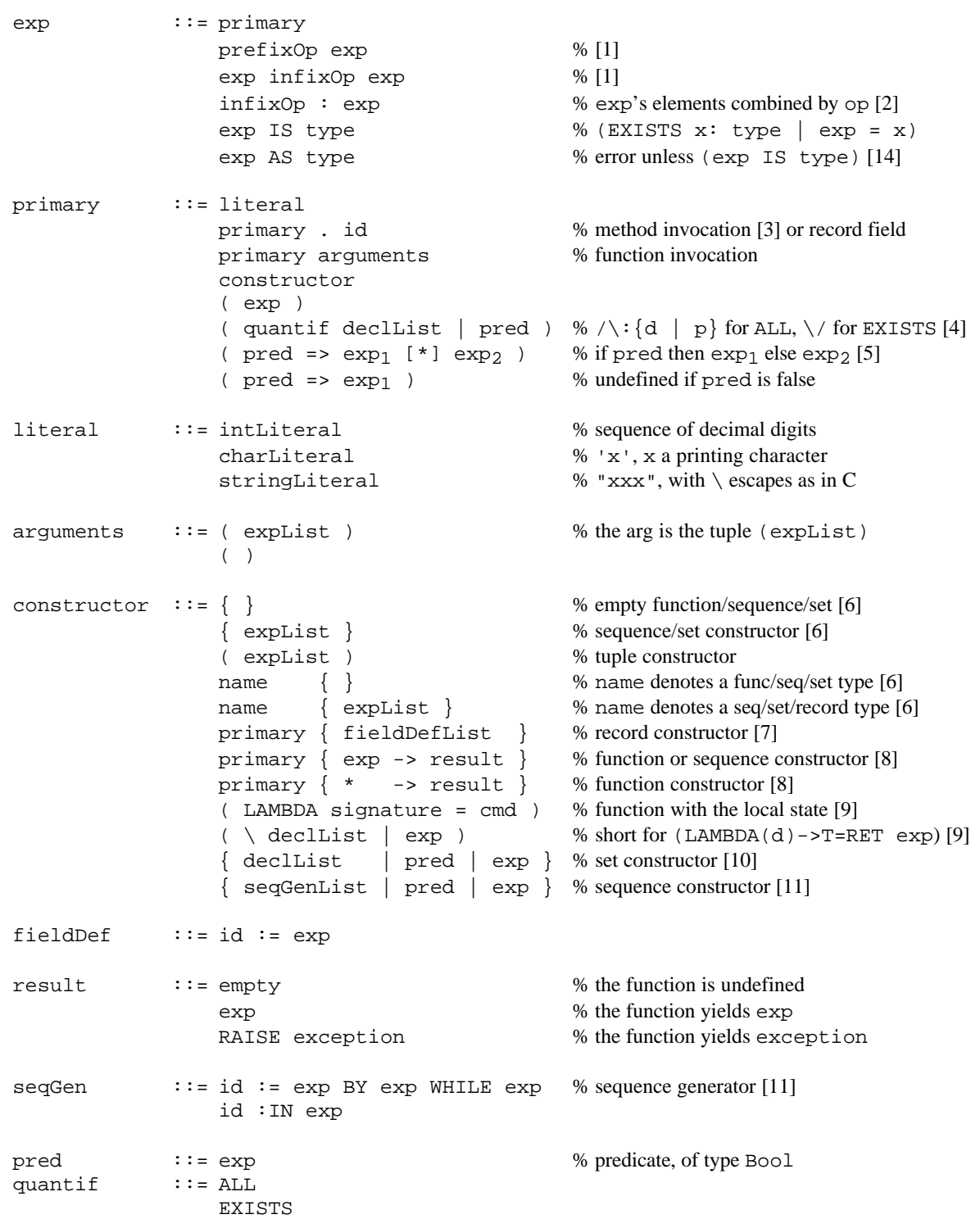




\begin{tabular}{|c|c|c|c|c|}
\hline \multicolumn{2}{|c|}{ (precedence) } & \multicolumn{2}{|l|}{ argument/result types } & \multirow{2}{*}{$\begin{array}{l}\text { operation } \\
\text { exponentiate }\end{array}$} \\
\hline$:=\star \star$ & $\%(8)$ & (Int, Int) $\rightarrow$ Int & & \\
\hline * & $\%(7)$ & $($ Int, Int $)->$ Int & & multiply \\
\hline & $\%$ & $(\mathrm{~T}->\mathrm{U}, \mathrm{U}->\mathrm{V})->(\mathrm{T}->\mathrm{V})$ & [12] & function composition \\
\hline / & $\%(7)$ & (Int, Int) $->$ Int & & divide \\
\hline // & $\%(7)$ & (Int, Int) $->$ Int & & remainder \\
\hline+ & $\%(6)$ & (Int, Int) $->$ Int & & add \\
\hline & $\%$ & $(\operatorname{SEQ} T, \operatorname{SEQ} T)->\operatorname{SEQ} T$ & {$[12]$} & concatenation \\
\hline & $\%$ & $(\mathrm{~T}->\mathrm{U}, \quad \mathrm{T}->\mathrm{U}) \rightarrow(\mathrm{T}->\mathrm{U})$ & {$[12]$} & function overlay \\
\hline - & $\%(6)$ & (Int, Int) $->$ Int & & subtract \\
\hline & $\%$ & $(\operatorname{SET} T, \operatorname{SET} T) \rightarrow$ SET $T$ & {$[12]$} & set difference; \\
\hline & $\%$ & (SEQ T, SEQ T) $\rightarrow$ SEQ T & [12] & multiset difference \\
\hline$!$ & $\%(6)$ & $(\mathrm{T}->\mathrm{U}, \mathrm{T})->\mathrm{B} \circ \mathrm{Ol}$ & [12] & function is defined \\
\hline$! !$ & $\%(6)$ & $(\mathrm{T}->\mathrm{U}, \mathrm{T})->\mathrm{B} \circ \mathrm{Ol}$ & [12] & func has normal value \\
\hline . & $\%(5)$ & (Int, Int) $->\mathrm{SEQ}$ Int & [12] & subrange \\
\hline$<=$ & $\%(4)$ & (Int, Int)->Bool & & less than or equal \\
\hline & $\%$ & $(\operatorname{SET} T, \operatorname{SET} T)->$ BOOl & [12] & subset \\
\hline & $\%$ & $(\operatorname{SEQ} T, \quad S E Q T)->$ BOol & {$[12]$} & prefix \\
\hline$<$ & $\begin{array}{l}\%(4) \\
\%\end{array}$ & $\begin{array}{c}(\mathrm{T}, \mathrm{T})->\mathrm{B} o o 1, \mathrm{~T} \text { with } \\
\mathrm{e} 1<\mathrm{e} 2=(\mathrm{e} 1<=\mathrm{e} 2\end{array}$ & $\begin{array}{l}<= \\
\mathrm{e} 1 \# \mathrm{e} 2)\end{array}$ & less than \\
\hline$>$ & $\%(4)$ & $\begin{array}{l}(\mathrm{T}, \mathrm{T})->\text { Bool, } \mathrm{T} \text { with } \\
\mathrm{e} 1>\mathrm{e} 2=\mathrm{e} 2<\mathrm{e} 1\end{array}$ & $<=$ & greater than \\
\hline$>=$ & $\begin{array}{l}\%(4) \\
\%\end{array}$ & $\begin{array}{l}(\mathrm{T}, \mathrm{T})->\text { Bool, } \mathrm{T} \text { with } \\
\mathrm{e} 1>=\mathrm{e} 2=\mathrm{e} 2<=\mathrm{e} 1\end{array}$ & $<=$ & greater or equal \\
\hline$=$ & $\%(4)$ & (Any, Any) $->$ Bool & [1] & equal \\
\hline \# & $\begin{array}{l}\%(4) \\
\%\end{array}$ & $\begin{array}{l}(\text { Any, Any })->\text { Bool } \\
\text { e1\#e } 2=\sim(e 1=e 2)\end{array}$ & & not equal \\
\hline$<<=$ & $\%(4)$ & $(\mathrm{SEQ} T, \mathrm{SEQ} T)->$ Bool & {$[12]$} & non-contiguous sub-sec \\
\hline IN & $\%(4)$ & $(\mathrm{T}$, SET T $)->$ Bool & [12] & membership \\
\hline 八 & $\%(2)$ & (Bool, Bool)->Bool & [13] & conditional and \\
\hline & $\%$ & $(\operatorname{SET} T, \operatorname{SET} T) \rightarrow$ SET $T$ & [12] & intersection \\
\hline$\backslash /$ & $\%(1)$ & $(\mathrm{B} \circ \circ 1, \mathrm{~B} \circ \circ 1)->\mathrm{B} \circ \circ \mathrm{l}$ & [13] & conditional or \\
\hline & $\%$ & (SET $T, \operatorname{SET} T$ T) $>$ SET $T$ & [12] & union \\
\hline$==>$ & $\%(0)$ & (Bool, Bool) $->$ Bool & [13] & conditional implies \\
\hline op & $\%(5)$ & not one of the above & {$[1]$} & \\
\hline- & $\%(6)$ & Int->Int & & negation \\
\hline$\sim$ & $\%(3)$ & Bool->Bool & & complement \\
\hline op & $\%(5)$ & not one of the above & [1] & \\
\hline
\end{tabular}

The ambiguity of the expression grammar is resolved by taking the infixops to be left associative and using the indicated precedences for the prefixops and infixops (with 8 for IS and AS and 5 for : or any operator not listed); higher numbers correspond to tighter binding. The precedence is determined by the operator symbol and doesn't depend on the operand types.

[1] The meaning of prefixop e is T. "prefixop" (e), where T is e's type, and of e1 infixop e2 is $\mathrm{T} 1$. "infixop" (e1, e2), where $\mathrm{T} 1$ is e1's type. The built-in types Int (and Nat with the same operations), Bool, sequences, sets, and functions have the operations given in the grammar. Section 9 on built-in methods specifies the operators for built-in types other than Int and Bool. Special case: e1 IN e2 means T2."IN" (e1, e2), where T2 is e2's type.

Note that the $=$ operator does not require that the types of its arguments agree, since both are Any. Also, = and \# cannot be overridden by WITH. To define your own abstract equality, use a different operator such as $"=="$.

[2] The exp must have type SEQ T or SET T. The value is the elements of exp combined into a single value by infixop, which must be associative and have an identity, and must also be commutative if exp is a set. Thus

$$
+:\{i \text { : Int }|0<i 八 i<5| i * * 2\}=1+4+9+16=30 \text {, }
$$

and if $s$ is a sequence of strings, $+: s$ is the concatenation of the strings. For another example, see the definition of quantifications in [4]. Note that the entire set is evaluated; see [10].

[3] Methods can be invoked by dot notation.

The meaning of e.id or e.id() is T.id(e), where T is e's type.

The meaning of e1.id(e2) is T.id(e1, e2), where T is e1's type.

Section 9 on built-in methods gives the methods for built-in types other than Int and Bool.

[4] A quantification is a conjunction (if the quantifier is ALL) or disjunction (if it is EXISTS) of the pred with the id's in the decllist bound to every possible value (that is, every value in their types); see section 4 for decl. Precisely, (ALL d $\mid p)=八:\{d \mid p\}$ and $($ EXISTS $d \mid p)=V /:\{d \mid p\}$. All the expressions in these expansions are evaluated, unlike $\mathrm{e} 2$ in the expressions e1 $\bigwedge$ e2 and e1 $\backslash /$ e2 (see [10] and [13]).

[5] A conditional (pred => e1 $\left[^{*}\right]$ e2) is not exactly an invocation. If pred is true, the result is the result of e1 even if e2 is undefined or exceptional; if pred is false, the result is the result of e2 even if e1 is undefined or exceptional. If pred is undefined, so is the result; if pred raises an exception, that is the result. If $\left[^{*}\right] \mathrm{e} 2$ is omitted and pred is false, the result is undefined.

[6] In a constructor \{expList \} each exp must have the same type $\mathrm{T}$, the type of the constructor is ( $\operatorname{SEQ} T+\operatorname{SET} T$ ), and its value is the sequence containing the values of the exps in the given order, which can also be viewed as the set containing these values.

If expList is empty the type is the union of all function, sequence and set types, and the value is the empty sequence or set, or a function undefined everywhere. If desired, these constructors can be prefixed by a name denoting a suitable set or sequence type.

A constructor $T\{e 1, \ldots, e n\}$, where $T$ is a record type $[f 1: T 1, \ldots, f n: T n]$, is short for a record constructor (see [7]) $\mathrm{T}\{\mathrm{f} 1:=\mathrm{e} 1, \ldots, \mathrm{fn}:=\mathrm{en}\}$.

[7] The primary must have a record type, and the constructor has the same type as its primary and denotes the same value except that the fields named in the fieldDefList have the given 
values. Each value must fit the type declared for its id in the record type. The primary may also denote a record type, in which case any fields missing from the fieldDefList are given arbitrary (but deterministic) values. Thus if $R=[a$ : Int, $b:$ Int $], R\{a:=3, b:=4\}$ is a record of type $R$ with $a=3$ and $b=4$, and $R\{a:=3, b:=4\}\{a:=5\}$ is a record of type $R$ with $a=5$ and $b=4$. If the record type is qualified by a Іеснтнат, the fields get values that satisfy it, and the constructor is undefined if that's not possible.

[8] The primary must have a function or sequence type, and the constructor has the same type as its primary and denotes a value equal to the value denoted by the primary except that it maps the argument value given by exp (which must fit the domain type of the function or sequence) to result (which must fit the range type if it is an exp). For a function, if result is empty the constructed function is undefined at exp, and if result is RAISE exception, then exception must be in the RAISES set of primary's type. For a sequence result must not be empty or RAISE, and exp must be in primary.dom or the constructor expression is undefined.

In the * form the primary must be a function type or a function, and the value of the constructor is a function whose result is result at every value of the function's domain type (the type on the left of the $->)$. Thus if $F=($ Int $->$ Int $)$ and $f=F\left\{{ }^{*}>0\right\}$, then $f$ is zero everywhere and $f\{4->1\}$ is zero except at 4 , where it is 1 . If this value doesn't have the function type, the constructor is undefined; this can happen if the type has a suснтнAт clause. For example, the type can't be a sequence.

[9] A LAMBDA constructor is a statically scoped function definition. When it is invoked, the meaning of the body is determined by the local state when the LAMBDA was evaluated and the global state when it is invoked; this is ad-hoc but convenient. See section 7 for signature and section 6 for cmd. The returns in the signature may not be empty. Note that a function can't have side effects.

The form ( $\backslash$ decllist $\mid$ exp) is short for (LAMBDA (declList) $\rightarrow T=$ RET exp), where $T$ is the type of exp. See section 4 for decl.

[10] A set constructor \{ decllist | pred | exp \} has type SET T, where exp has type T in the current state augmented by declList; see section 4 for decl. Its value is a set that contains $\mathrm{x}$ iff (EXISTS declList $\mid$ pred $八 \mathrm{x}=\exp$ ). Thus

$\{i$ : Int $|0<i 八 i<5| i * * 2\}=\{1,4,9,16\}$

and both have type SET Int. If pred is omitted it defaults to true. If $\mid$ exp is omitted it defaults to the last id declared:

$\{i$ : Int $\mid 0<i 八 i<5\}=\{1,2,3,4\}$

Note that if $\mathrm{s}$ is a set or sequence, IN $\mathrm{s}$ is a type (see section 4), so you can write a constructor like $\{i: I N$ s $\mid i>4\}$ for the elements of s greater than 4 . This is shorter and clearer than

$$
\text { \{i } \mid \text { i IN } s \text { 八 } i>4
$$

If there are any values of the declared id's for which pred is undefined, or pred is true and exp is undefined, then the result is undefined. If nothing is undefined, the same holds for exceptions; if more than one exception is raised, the result exception is an arbitrary choice among them.

[11] A sequence constructor \{ seqGenList | pred | exp \} has type SEQ T, where exp has type $\mathrm{T}$ in the current state augmented by seqGenList, as follows. The value of

$\{\mathrm{x} 1:=$ e01 BY e1 WHILE p1, .., xn := e0n BY en WHILE pn $\mid$ pred $\mid$ exp $\}$

is the sequence which is the value of result produced by the following program. Here exp has type $\mathrm{T}$ and result is a fresh identifier (that is, one that doesn't appear elsewhere in the program). There's an informal explanation after the program.

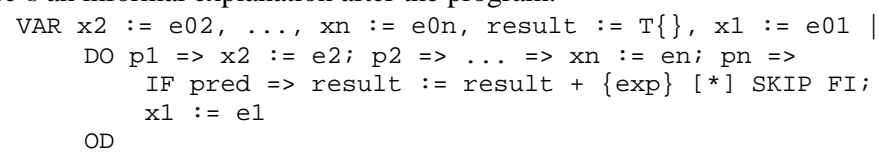

However, $e 0 i$ and $e i$ are not allowed to refer to $\times j$ if $j>i$. Thus the $n$ sequences are unrolled in parallel until one of them ends, as follows. All but the first are initialized; then the first is initialized and all the others computed, then all are computed repeatedly. In each iteration, once all the $\mathrm{xi}$ have been set, if pred is true the value of exp is appended to the result sequence; thus pred serves to filter the result. As with set constructors, an omitted pred defaults to true, and an omitted $\mid$ exp defaults to $\mid$ xn. An omitted whILE pi defaults to whILE true. An omitted $:=\mathrm{e} 0 \mathrm{i}$ defaults to

$:=\{x:$ Ti $\mid$ true $\}$.choose

where $\mathrm{Ti}$ is the type of $\mathrm{e} i$; that is, it defaults to an arbitrary value of the right type.

The generator $\mathrm{xi}$ : IN ei generates the elements of the sequence ei in order. It is short for $j:=0$ BY $j+1$ WHILE $j<$ ei.size, xi BY ei(j)

where $j$ is a fresh identifier. Note that if the :IN isn't the first generator then the first element of ei is skipped, which is probably not what you want. Note that : IN in a sequence constructor overrides the normal use of IN $\mathrm{s}$ as a type (see [10]).

Undefined and exceptional results are handled the same way as in set constructors.

Examples

$$
\begin{aligned}
& \{i:=0 \text { BY } i+1 \text { WHILE } i<=n\} \\
& (r:=\text { head BY r.next WHILE } r \# \text { nil | | r.val }\} \\
& \{x: I N \text { s, sum }:=0 \text { BY sum }+x\} \\
& \{x: I N \text { s, sum }:=0 \text { BY sum }+x\} \text {.last } \\
& \{x: I N \text { s, rev }:=\{\} \text { BY }\{x\}+\text { rev }\} \text {. last } \\
& \{x: I N s \mid f(x)\} \\
& \{i: I N 1 \ldots n|i / / 2 \# 0| i * i\} \\
& \{i: I N 1 \ldots n, i t e r:=\text { e BY } f(i t e r)\}
\end{aligned}
$$

$=0 \ldots \mathrm{n}=\{0,1, \ldots, \mathrm{n}\}$ the val fields of a list starting at head partial sums of $s$ $+: s$, the last partial sum reverse of $s$

$s * f$

squares of odd numbers $<=\mathrm{n}$ $\left\{f(e), f^{2}(e), \ldots, f^{n}(e)\right.$

[12] These operations are defined in section 9 .

[13] The conditional logical operators are defined in terms of conditionals:

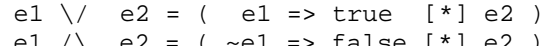

$$
\text { e1 }==e^{2}=\left(\sim e 1 \Rightarrow \text { true }\left[{ }^{*}\right] \text { e } 2\right)
$$

Thus the second operand is not evaluated if the value of the first one determines the result.

[14] AS changes only the type of the expression, not its value. Thus if (exp IS type) the value of (exp AS type) is the value of exp, but its type is type rather than the type of exp. 


\section{Commands}

A command changes the state (or does nothing). Recall that the state is a mapping from names to values; we denote it by state. Commands are non-deterministic. An atomic command is one that is inside $<<\ldots .>>$ brackets.

The meaning of an atomic command is a set of possible transitions (that is, a relation) between a state and an outcome (a state plus an optional exception); there can be any number of outcomes from a given state. One possibility is a looping exceptional outcome. Another is no outcomes. In this case we say that the atomic command fails; this happens because all possible choices within it encounter a false guard or an undefined invocation.

If a subcommand fails, an atomic command containing it may still succeed. This can happen because it's one operand of [ ] or [*] and the other operand succeeds. If can also happen because a non-deterministic construct in the language that might make a different choice. Leaving exceptions aside, the commands with this property are [ ] and VAR (because it chooses arbitrary values for the new variables). If we gave an operational semantics for atomic commands, this situation would correspond to backtracking. In the relational semantics that we actually give (in Atomic Semantics of Spec), it corresponds to the fact that the predicate defining the relation is the "or" of predicates for the subcommands. Look there for more discussion of this point.

A non-atomic command defines a collection of possible transitions, roughly one for each $<<\ldots>>$ command that is part of it. If it has simple commands not in atomic brackets, each one also defines a possible transition, except for assignments and invocations. An assignment defines two transitions, one to evaluate the right hand side, and the other to change the value of the left hand side. An invocation defines a transition for evaluating the arguments and doing the call and one for evaluating the result and doing the return, plus all the transitions of the body These rules are somewhat arbitrary and their details are not very important, since you can always write separate commands to express more transitions, or atomic brackets to express fewer transitions. The motivation for the rules is to have as many transitions as possible, consistent with the idea that an expression is evaluated atomically.

A complete collection of possible transitions defines the possible sequences of states or histories there can be any number of histories from a given state. A non-atomic command still makes choices, but it does not backtrack and therefore can have histories in which it gets stuck, even though in other histories a different choice allows it to run to completion. For the details, see handout 17 on formal concurrency.

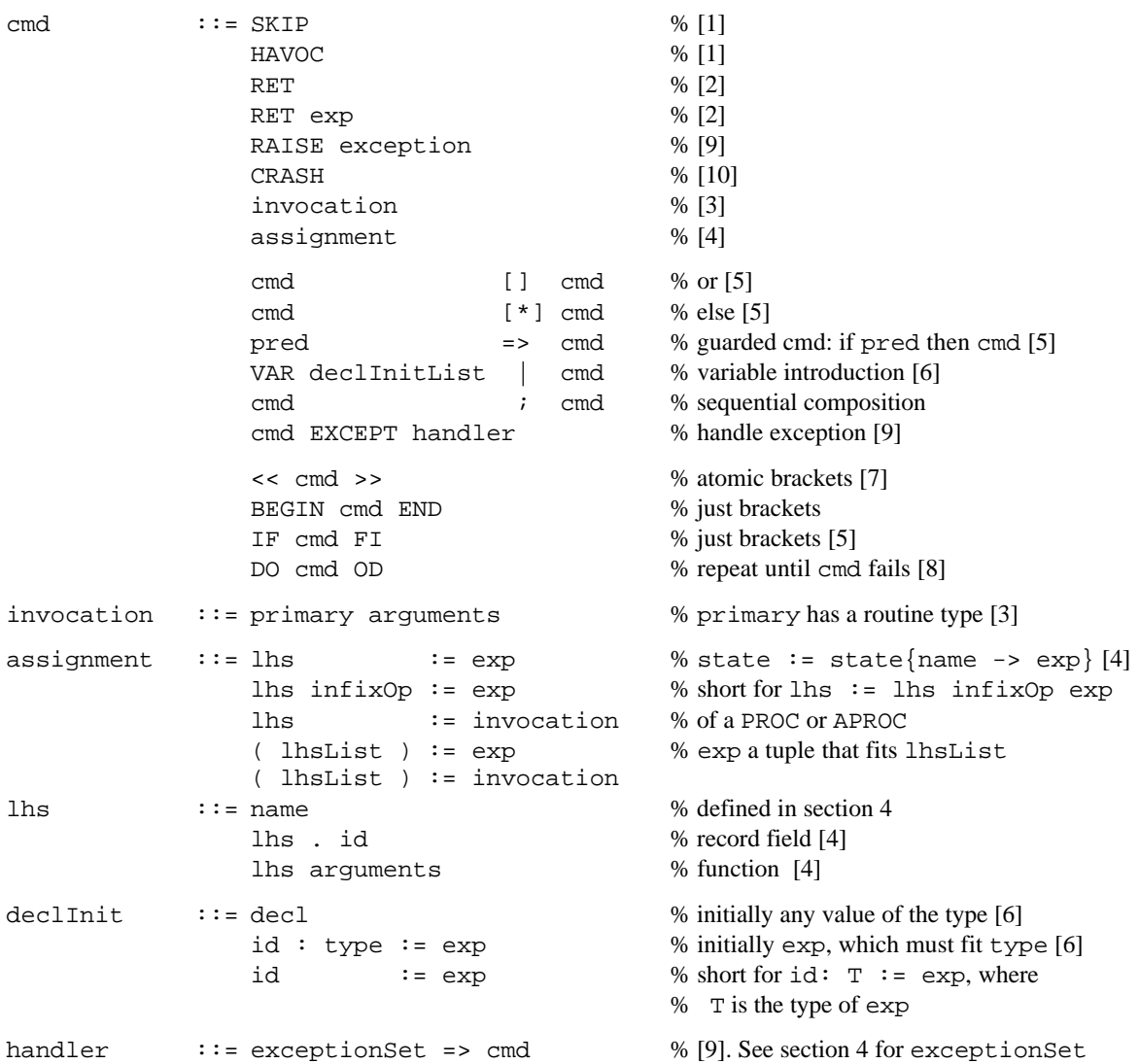

$\%$ [9]. See section 4 for exceptionset

The ambiguity of the command grammar is resolved by taking the command composition operations ; , [ ] , and [*] to be left-associative and EXCEPT to be right associative, and giving [] and [*] lowest precedence, $=>$ and $\mid$ next (to the right only, since their left operand is an exp), ; next, and EXCEPT highest precedence.

[1] The empty command and SKIP make no change in the state. HAVOC produces an arbitrary outcome from any state; if you want to specify undefined behavior when a precondition is not satisfied, write $\sim$ precondition $\Rightarrow$ HAVOC.

[2] A RET may only appear in a routine body, and the exp must fit the result type of the routine The exp is omitted iff the returns of the routine's signature is empty.

[3] For arguments see section 5. The argument are passed by value, that is, assigned to the formals of the procedure A function body cannot invoke a PROC Or APROC; together with the rule for assignments (see [7]) this ensures that it can't affect the state. An atomic command can invoke an APROC but not a PROC. A command is atomic iff it is $<<$ cmd $>>$, a subcommand of an 
atomic command, or one of the simple commands SKIP, HAVOC, RET, or RAISE. The typechecking rule for invocations is the same as for function invocations in expressions.

[4] You can only assign to a name declared with VAR or in a signature. In an assignment the exp must fit the type of the $1 \mathrm{hs}$, or there is a fatal error. In a function body assignments must be to names declared in the signature or the body, to ensure that the function can't have side effects. An assignment to a left hand side that is not a name is short for assigning a constructor to a name. In particular,

lhs (arguments) := exp is short for lhs := lhs $\{$ arguments->exp $\}$, and

lhs . id $\quad:=\exp$ is short for $l \mathrm{hs}:=\operatorname{lhs}\{$ id $:=\exp \}$

These abbreviations are expanded repeatedly until $\mathrm{lhs}$ is a name.

In an assignment the right hand side may be an invocation (of a procedure) as well as an ordinary expression (which can only invoke a function). The meaning of 1 hs $:=$ exp or

lhs := invocation is to first evaluate the exp or do the invocation and assign the result to a temporary variable $\mathrm{v}$, and then do $\mathrm{lhs}:=\mathrm{v}$. Thus the assignment command is not atomic unless it is inside $\langle<\ldots>>$.

If the left hand side of an assignment is a (lhslist), the exp must be a tuple of the same length, and each component must fit the type of the corresponding 1hs. Note that you cannot write a tuple constructor that contains procedure invocations.

[5] A guarded command fails if the result of pred is undefined or false. It is equivalent to $\mathrm{cmd}$ if the result of pred is true. A pred is just a Boolean exp; see section 4.

S1 [ ] S2 chooses one of the Si to execute. It chooses one that doesn't fail. Usually S1 and S2 will be guarded. For example,

$\mathrm{x}=1 \Rightarrow \mathrm{y}:=0$ [] $\mathrm{x}>1 \Rightarrow \mathrm{y}:=1$ sets $\mathrm{y}$ to 0 if $\mathrm{x}=1$, to 1 if $\mathrm{x}>1$, and has no outcome if $\mathrm{x}<1$. But $\mathrm{x}=1 \Rightarrow \mathrm{y}:=0[] \quad \mathrm{x}>=1 \Rightarrow \mathrm{y}:=1$ might set $\mathrm{y}$ to 0 or 1 if $\mathrm{x}=1$.

S1 [*] S2 is the same as S1 unless S1 fails, in which case it's the same as S2.

IF ... FI are just command brackets, but it often makes the program clearer to put them around a sequence of guarded commands, thus:

$$
\begin{aligned}
& \text { IF } x<0 \Rightarrow y:=3 \\
& {[] \quad x=0 \Rightarrow y:=4} \\
& \text { [*] }
\end{aligned}
$$

6] In a VAR the unadorned form of declInit initializes a new variable to an arbitrary value of the declared type. The $:=$ form initializes a new variable to exp. Precisely,

$$
\text { VAR id: } T:=\exp \mid \mathrm{C}
$$

is equivalent to

VAR id: $\mathrm{T}$ | id := exp; $c$

The exp could also be a procedure invocation, as in an assignment.

Several declinits after VAR is short for nested VARs. Precisely,

$$
\text { VAR declinit, declinitlist } \mid \text { cmd }
$$

is short for

VAR declinit | VAR declInitlist | cmd

This is unlike a module, where all the names are introduced in parallel.

[7] In an atomic command the atomic brackets can be used for grouping instead of BEGIN ... END; since the command can't be any more atomic, they have no other meaning in this context.
[8] Execute $c m d$ repeatedly until it fails. If $\mathrm{cmd}$ never fails, the result is a looping exception that doesn't have a name and therefore can't be handled. Note that this is not the same as failure.

[9] Exception handling is as in Clu, but a bit simplified. Exceptions are named by literal strings (which are written without the enclosing quotes). A module can also declare an identifier that denotes a set of exceptions. A command can have an attached exception handler, which gets to look at any exceptions produced in the command (by RAISE or by an invocation) and not handled closer to the point of origin. If an exception is not handled in the body of a routine, it is raised by the routine's invocation.

An exception ex must be in the RAISES set of a routine $r$ if either RAISE ex or an invocation of routine with ex in its RAISES set occurs in the body of $r$ outside the scope of a handler for ex.

[10] CRASH stops the execution of any current invocations in the module other than the one that executes the CRASH, and discards their local state. The same thing happens to any invocations outside the module from within it. After CRASH, no procedure in the module can be invoked from outside until the routine that invokes it returns. CRASH is meant to be invoked from within a special Crash procedure in the module that models the effects of a failure.

\section{Modules}

A program is some global declarations plus a set of modules. Each module contains variable routine, exception, and type declarations.

Module definitions can be parameterized with mformals after the module id, and a parameterized module can be instantiated. Instantiation is like macro expansion: the formal parameters are replaced by the arguments throughout the body to yield the expanded body. The parameters must be types, and the body must type-check without any assumptions about the argument that replaces a formal other than the presence of a WITH clause that contains all the methods mentioned in the formal parameter list (that is, formals are treated as distinct from all other types).

Each module is a separate scope, and there is also a Global scope for the identifiers declared at the top level of the program. An identifier id declared at the top level of a non-parameterized module $\mathrm{m}$ is short for $\mathrm{m}$.id when it occurs in $\mathrm{m}$. If it appears in the exports, it can be denoted by $\mathrm{m}$.id anywhere. When an identifier id that is declared globally occurs anywhere, it is short for Global.id. Global cannot be used as a module id.

An exported id must be declared in the module. If an exported id has a WITH clause, it must be declared in the module as a type with at least those methods, and only those methods are accessible outside the module; if there is no WITH clause, all its methods and constructors are accessible. This is Spec's version of data abstraction. 


\begin{tabular}{|c|c|c|}
\hline program & $::=$ toplevel* module* END & \\
\hline module & $::=$ modclass id mformals exports & $=$ body END id \\
\hline modclass & $::=$ MODULE & $\%[4]$ \\
\hline $\begin{array}{l}\text { exports } \\
\text { export }\end{array}$ & $\begin{aligned}:: & =\text { EXPORT exportList } \\
::= & \text { id } \\
& \text { id WITH }\{\text { methodList }\}\end{aligned}$ & $\%$ see section 4 for method \\
\hline mformals & $\begin{aligned}::= & \text { empty } \\
& {[\text { mfpList }] }\end{aligned}$ & \\
\hline $\operatorname{mfp}$ & $\begin{aligned}::= & \text { id } \\
& \text { id WITH }\{\text { declList }\}\end{aligned}$ & $\begin{array}{l}\% \text { module formal parameter } \\
\% \text { see section } 4 \text { for decl }\end{array}$ \\
\hline body & $\begin{aligned}::= & \text { toplevel* } \\
& \text { id [ typelist ] }\end{aligned}$ & $\begin{array}{l}\% \text { id must be the module id } \\
\% \text { instance of parameterized module }\end{array}$ \\
\hline toplevel & $\begin{aligned}::= & \text { VAR declinit* } \\
& \text { CONST declInit* } \\
& \text { routineDecl } \\
& \text { EXCEPTION exSetDecl* } \\
& \text { TYPE typeDecl* }\end{aligned}$ & $\begin{array}{l}\% \text { declares the decl ids [1] } \\
\% \text { declares the decl ids as constant } \\
\% \text { declares the routine id } \\
\% \text { declares the exception set ids } \\
\% \text { declares the type ids and any } \\
\% \text { ids in ENUMs }\end{array}$ \\
\hline routineDecl & $\begin{aligned}::= & \text { FUNC id signature }=\mathrm{cmd} \\
& \text { APROC id signature }=<<\mathrm{cmd}>> \\
& \text { PROC id signature }=\mathrm{cmd} \\
& \text { THREAD id signature }=\mathrm{cmd}\end{aligned}$ & $\begin{array}{l}\% \text { function } \\
\% \text { atomic procedure } \\
\% \text { non-atomic procedure } \\
\% \text { one thread for each possible } \\
\% \text { invocation of the routine }[2]\end{array}$ \\
\hline signature & 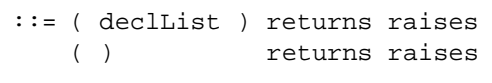 & $\begin{array}{l}\% \text { see section } 4 \text { for returns } \\
\% \text { and raises }\end{array}$ \\
\hline exSetDecl & $::=i d=$ exceptionset & $\%$ see section 4 for exceptionset \\
\hline typeDecl & $\begin{aligned}::=i d & =\text { type } \\
i d & =\text { ENUM }[\text { idList }]\end{aligned}$ & $\begin{array}{l}\% \text { see section } 4 \text { for type } \\
\% \text { a value is one of the id's [3] }\end{array}$ \\
\hline
\end{tabular}

[1] The ": = exp" in a declInit (defined in section 6) specifies an initial value for the variable The exp is evaluated in a state in which each variable used during the evaluation has been initialized, and the result must be a normal value, not an exception. The exp sees all the names known in the scope, not just the ones that textually precede it, but the relation "used during evaluation of initial values" on the variables must be a partial order so that initialization makes sense. As in an assignment, the exp may be a procedure invocation as well as an ordinary expression. It's a fatal error if the exp is undefined or the invocation fails.

[2] Instead of being invoked by the client of the module or by another procedure, a thread is automatically invoked in parallel once for every possible value of its arguments. The thread is named by the id in the declaration together with the argument values. So

VAR sum $:=0$, count $:=0$
THREAD P $(i:$ Int $)=i$ IN $0 \ldots 9 \Rightarrow$

$\operatorname{VAR} t \mid t:=\mathrm{F}(i) ;<<$ sum $:=$ sum $+\mathrm{t}>>$; $<<$ count $:=$ count $+1>>$ adds up the values of $F(0) \ldots F(9)$ in parallel. It creates a thread $P(i)$ for every integer $i$; the threads $P(0), \ldots, P(9)$ for which the guard is true invoke $F(0), \ldots, F(9)$ in parallel and total the results in sum. When count $=10$ the total is complete.

A thread is the only way to get an entire program to do anything (except evaluate initializing expressions, which could have side effects), since transitions only happen as part of some thread.

[3] The id's in the list are declared in the module; their type is the Enum type. There are no operations on enumeration values except the ones that apply to all types: equality, assignment, and routine argument and result communication.

[4] A class is shorthand for a module that declares a convenient object type. The next few paragraphs specify the shorthand, and the last one explains the intended usage.

If the class $i d$ is $O b j$, the module $i d$ is ObjMod. Each variable declared in a top level VAR in the class becomes a field of the objRec record type in the module. The module exports only a type $\mathrm{Obj}$ that is also declared globally. obj indexes a collection of state records of type objRec stored in the module's objs variable, which is a function Obj->ObjRec. Obj's methods are all the names declared at top level in the class except the variables, plus the new method described below; the exported obj's methods are all the ones that the class exports plus new.

To make a class routine suitable as a method, it needs access to an objRec that holds the state of the object. It gets this access through a self parameter of type obj, which it uses to refer to the object state objs (self). To carry out this scheme, each routine in the module, unless it appears in a WITH clause in the class, is 'objectified' by giving it an extra self parameter of type obj. In addition, in a routine body every occurrence of a variable $\mathrm{v}$ declared at top level in the class is replaced by objs (self) . v in the module, and every invocation of an objectified class routine gets self as an extra first parameter.

The module also gets a synthesized and objectified stdNew procedure that adds a state record to objs, initializes it from the class's variable initializations (rewritten like the routine bodies), and returns its $\mathrm{Ob}_{j}$ index; this procedure becomes the new method of obj unless the class already has a new routine.

\section{A class cannot declare a THREAD.}

The effect of this transformation is that a variable obj of type obj behaves like an object. The state of the object is objs (obj). The invocation obj.m or obj.m(x) is short for objMod.m (obj) or objMod.m(obj, x) by the usual rule for methods, and it thus invokes the method m; in m's body each occurrence of a class variable refers to the corresponding field in obj's state. obj.new () returns a new and initialized obj object. The following example shows how a class is transformed into a module. 
CLASS Obj EXPORT T1, f, p, ... =

TYPE T1 $=\ldots$ WITH $\{$ add $:=$ AddT $\}$

CONST $\mathrm{C}:=$

MODULE ObjMOd EXPORT Obj WITH $\{$ TI, f, p, new $\}=$

TYPE T1 $=\ldots$ WITH $\quad\{$ add $:=$ AddT $\}$

CONST $\mathrm{C}:=\ldots$

VAR V1:T1:=ei, v2:T2:=pi(v1), .. TYPE ObjRec $=[\mathrm{v} 1: \mathrm{T} 1, \mathrm{v} 2: \mathrm{T} 2, \ldots]$

$\mathrm{Obj}=$ Int $\mathrm{WITH}\{\mathrm{T} 1, \mathrm{c}, \mathrm{f}:=\mathrm{f}, \mathrm{p}:=\mathrm{p}$,

$V A R$ objs: Obj $\rightarrow$ ObjRec $:=(1)$

FUNC $\mathrm{f}(\mathrm{p} 1: \mathrm{RT} 1, \ldots)=\ldots \mathrm{v} 1 \ldots$ PROC $\mathrm{p}(\mathrm{p} 2: \mathrm{RT} 2, \ldots)=\ldots \mathrm{v} 2 \ldots$

FUNC AddT $(t 1, t 2)=\ldots$

FUNC $f($ self: Obj, p1: RT1, ...) = ... objs(self).v1 ... PROC p(self: Obj, p2: RT2, ...) = ... objs(self).v2 ... FUNC AddT $(t 1, t 2)=\ldots \quad \%$ in T1's WITH, so not objectified

PROC StdNew(self: obj) $\rightarrow$ Obj $=$

VAR obj: obj | obj IN objs.dom => objs (obj) : $:=$ objRec \{\}

pi (objs (obj) .v1)

END ObjMod

TYPE Obj $=$ ObjMod.Obj

In abstraction functions and invariants we also write obj.n for field $\mathrm{n}$ in obj's state, that is, for objMod.objs (obj).n.

\section{Scope}

The declaration of an identifier is known throughout the smallest scope in which the declaration appears (redeclaration is not allowed). This section summarizes how scopes work in Spec; terms defined before section 7 have pointers to their definitions. A scope is one of

the whole program, in which just the predefined (section 3), module, and globally declared identifiers are declared;

a module;

the part of a routineDecl or LAMBDA expression (section 5) after the =;

the part of a VAR declInit | cmd command after the | (section 6);

the part of a constructor or quantification after the first $\mid$ (section 5).

a record type or methodDeflist (section 4);

An identifier is declared by

a module id, mfp, or toplevel (for types, exception sets, ENUM elements, and named routines),

a decl in a record type (section 4), | constructor or quantification (section 5), declinit (section 6), routine signature, or WITH clause of a mfp, or

a methodDef in the WITH clause of a type (section 4).

An identifier may not be declared in a scope where it is already known. An occurrence of an identifier id always refers to the declaration of id which is known at that point, except when id is being declared (precedes a $:$, the $=$ of a toplevel, the $:=$ of a record constructor, or the $:=$ or BY in a segGen), or follows a dot. There are four cases for dot:

moduleId . id - the id must be exported from the basic module moduleId, and this expression denotes the meaning of id in that module.

record . id - the id must be declared as a field of the record type, and this expression denotes that field of record. In an assignment's 1 hs see [7] in section 6 for the meaning.

typeId . id - the typeId denotes a type, id must be a method of this type, and this expression denotes that method.

primary . id - the id must be a method of primary's type, and this expression, together with any following arguments, denotes an invocation of that method; see [2] in section 5 on expressions.

If $i d$ refers to an identifier declared by a toplevel in the current module $\mathrm{m}$, it is short for $\mathrm{m}$. id. If it refers to an identifier declared by a toplevel in the program, it is short for Global.id.

Once these abbreviations have been expanded, every name in the state is either global (contains a dot and is declared in a toplevel), or local (does not contain a dot and is declared in some other way).

Exceptions look like identifiers, but they are actually string literals, written without the enclosing quotes for convenience. Therefore they do not have scope. 


\section{Built-in methods}

Some of the type constructors have built-in methods, among them the operators defined in the expression grammar. The built-in methods for types other than Int and Bool are defined below. Note that these are not complete definitions of the types; they do not include the constructors.

Sets

\section{A set has methods for}

computing union, intersection, and set difference, and adding or removing an element, testing for membership and subset,

choosing (deterministically) a single element from a set, or a sequence with the same members, or a maximum or minimum element, and turning a set into its characteristic predicate (the inverse is the predicate's set method).

We define these operations using a module that represents a set by its characteristic predicate. Precisely, SET T behaves as though it were Set [T] .S, where

\section{MODULE Set [T] EXPORT $S=$}

TYPE $S=$ AnY->Bool SUCHTHAT $(\backslash S \mid($ ALL any $\mid S($ any $)=\Rightarrow($ any IS T $))$ ) $\%$ Defined everywhere so that type inclusion will work; see section 4 . WITH $\{$ " $\backslash / ":=$ Union, $" \bigwedge ":=$ Intersection, $"-":=$ Difference,
"IN":=In, "<=":=Subset, choose:=Choose, seq:=Seq, pred:=Pred, perms:=Perms, fsort $:=$ FSort, sort:=Sort,

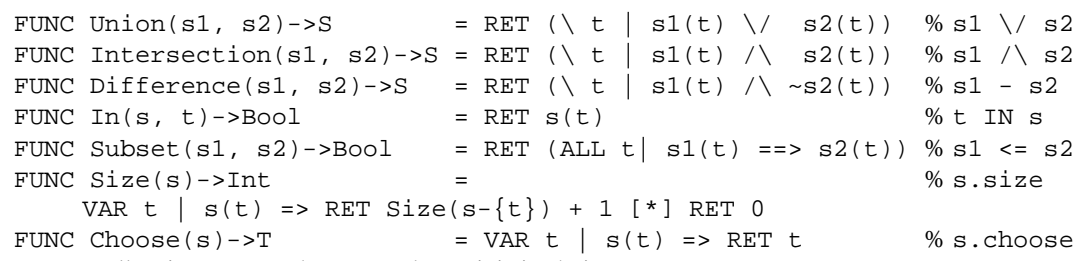

$\%$ s.perms
There are constructors \{\} for the empty set, $\{e 1, e 2, \ldots\}$ for a set with specific elements, and $\{$ decllist $\mid$ pred $\mid \exp \}$ for a set whose elements satisfy a predicate. These constructors are described in [6] and [10] of section 5. Note that $\{t \mid p\}$.pred $=(\backslash t \mid p)$, and similarly $(\backslash t$ p) . set $=\{t \mid p\}$.

\section{Functions}

The function types T->U and T->U RAISES XS have methods for

composition, overlay, inverse, and restriction;

testing whether a function is defined at an argument and whether it produces a normal (nonexceptional) result at an argument, and for the domain and range;

converting a function to a relation (the inverse is the relation's func method).

In other words, they behave as though they were Function $[T, U] . F$, where (making allowances for the fact that $\mathrm{XS}$ and $\mathrm{v}$ are pulled out of thin air):

\section{MODULE Function [T, U] EXPORT F =}

TYPE F $=$ T->U RAISES XS WITH $\{n * ":=$ Compose, $"+":=$ Overlay, inv:=Inverse, restrict:=Restrict, "! ":=Defined, "!!":=Normal,

$$
R=(T, U)->\text { Bool }
$$

FUNC Compose (f, $g: U \rightarrow V) \rightarrow(T \rightarrow V)=\operatorname{RET}(\backslash t \mid g(f(t)))$

FUNC Overlay (f1, f2) $\rightarrow F=\operatorname{RET}\left(\backslash t \mid\left(f 2 ! t \Rightarrow f 2(t)\left[{ }^{*}\right] f 1(t)\right)\right)$ $\%(f 1+f 2)$ is $f 2(x)$ if that is defined, otherwise $f 1(x)$

FUNC Inverse $(f) \rightarrow(U \rightarrow T)=$ RET f.rel.inv.func

FUNC Restrict (f, $s: \operatorname{SET} T) \rightarrow F=\operatorname{RET}(1) t \mid(t$ IN $s \Rightarrow f(t)))$

FUNC Defined $(f, t)->$ Bool $=$

IF $f(t)=f(t) \Rightarrow$ RET true [*] RET false FI EXCEPT XS $\Rightarrow$ RET true FUNC Normal $(f, t)->$ Bool $=$

$$
\text { IF } f(t)=f(t) \Rightarrow \text { RET true [*] RET false FI EXCEPT XS } \Rightarrow \text { RET false }
$$

FUNC Domain $(f) \rightarrow$ SET $T=R E T\{t \mid f ! t\}$

FUNC Range (f) $\rightarrow$ SET $U=\operatorname{RET}\{t|f ! ! t| f(t)\}$

FUNC Rel(f) $\rightarrow R=\operatorname{RET}(\backslash t, u \mid f(t)=u)$

END Function

Note that there are constructors \{\} for the function undefined everywhere, $T\left\{{ }^{*}->\right.$ result $\}$ for a function of type $T$ whose value is result everywhere, and $f\{\exp \rightarrow$ result $\}$ for a function which is the same as $f$ except at exp, where its value is result. These constructors are described in [6] and [8] of section 5. There are also lambda constructors for defining a function by a computation, described in [9] of section 5 . $\%$ s. sort; only if $\mathrm{T}$ has $<=$ $\%$ s.fmax (f); a max under $\mathrm{f}$ $\%$ s.fmin (f); a min under s.max; only if $\mathrm{T}$ has $<=$ $\% \mathrm{~s} . \mathrm{min}$; only if $\mathrm{T}$ has $<=$ 
A total function $\mathrm{T}->\mathrm{B} O \mathrm{Ol}$ is a predicate and has an additional method to compute the set of T's that satisfy the predicate (the inverse is the set's pred method). In other words, a predicate behaves as though it were Predicate $[T] . P$, where

MODULE Predicate [T] EXPORT P =

TYPE $P=T \rightarrow$ Bool WITH $\{$ set $:=$ Set $\}$

FUNC Set $(p) \rightarrow$ SET $T=\operatorname{RET}\{t \mid p(t)\}$

END Predicate

A predicate with $\mathrm{T}=(\mathrm{TO}, \mathrm{UO})$ is a relation and has additional methods to turn it into a

function, a total function, or a function to sets of UO's, and to get its domain and range, invert it or compose it (overriding the methods for a function). In other words, it behaves as though it were Relation [T0, U0].R, where (making allowances for the fact that $\mathrm{V}$ is pulled out of thin air in Compose):

\section{MODULE Relation [T, U] EXPORT R}

TYPE $R=(T, U) \rightarrow$ Bool WITH $\{$ func:=Func, totalF: $:=$ TotalFunc, setF $:=$ SetFunc, dom:=Domain, rng :=Range,

$$
\text { inv:=Inverse, } " \star n:=\text { Compose }
$$

FUNC Func $(r) \rightarrow(T \rightarrow U)=$

$\%$ The result function is defined at $t$ iff $r$ relates $t$ to a single $u$.

$$
\operatorname{RET}(\backslash t \mid(r \cdot \operatorname{set} F(t) \cdot \operatorname{size}=1 \Rightarrow r \cdot \operatorname{set} F(t) \text {. choose }))
$$

FUNC TotalFunc $(r) \rightarrow(T \rightarrow(U+N u l l))=$

$\%$ The result function is defined everywhere, returning some related $\mathrm{U}$, or $\mathrm{nil}$ if there is none. $\operatorname{RET}(\backslash t \mid(r \cdot \operatorname{setF}(t) \#\{\} \quad \Rightarrow r \cdot \operatorname{set} F(t)$. choose [*] nil))

FUNC SetFunc $(r) \rightarrow(T \rightarrow \operatorname{SET} U)=\operatorname{RET}(\backslash t \mid\{u \mid r(t, u)\})$

$\%$ The result function is defined everywhere, returning the set of related U's.

FUNC Domain $(r) \rightarrow \operatorname{SET} T=\operatorname{RET}\{t, u|r(t, u)| t\}$

FUNC Range $(r) \rightarrow \operatorname{SET} U=\operatorname{RET}\{t, u|r(t, u)| u\}$

FUNC Inverse $(r) \rightarrow((U, T) \rightarrow$ Bool $)=\operatorname{RET}(\backslash u, t \mid r(t, u))$

FUNC Compose $(r: R, S:(U, V)->$ Bool $) \rightarrow(T, V)->$ Bool $=$

$\operatorname{RET}(\backslash t, \mathrm{v} \mid(\operatorname{EXISTS} u \mid r(t, u) \wedge \mathrm{s}(\mathrm{u}, \mathrm{v})))$

END Relation

A relation with $\mathrm{T}=\mathrm{U}$ is a graph and has additional methods to test whether a sequence of $\mathrm{T}$ 's is a path in the graph and to compute the transitive closure . In other words, it behaves as though it were Graph $[\mathrm{T}] . \mathrm{G}$, where

\section{MODULE Graph [T] EXPORT G =}

TYPE G $=(\mathrm{T}, \mathrm{T}) \rightarrow$ Bool WITH $\{$ isPath:=IsPath, closure:=TransitiveClosure $P=\operatorname{SEQ} T$

FUNC IsPath $(g, p)=\operatorname{RET}(\operatorname{ALL} i: \operatorname{IN} p \cdot \operatorname{dom}-\{0\} \mid g(p(i-1), p(i))$ )

$\%$ Any $\mathrm{p}$ of size $<=1$ is a path by this definition.

FUNC Transitiveclosure $(g) \rightarrow G=\operatorname{RET}(\backslash t 1, t 2$

$($ EXISTS $\mathrm{p} \mid \mathrm{p} \cdot$ size $>1$ 八 p.head $=\mathrm{t} 1$ 八p.last $=\mathrm{t} 2$ 八 g.isPath $(\mathrm{p})$ ) $)$

END Graph

\section{Sequences}

A function is called a sequence if its domain is a finite set of consecutive Int's starting at 0 , that is, if it has type

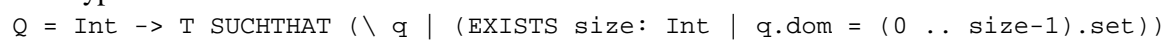

We denote this type (with the methods defined below) by SEQ T. A sequence inherits the methods of the function (though it overrides + ), and it also has methods for

detaching or attaching the first or last element,

extracting a segment of a sequence, concatenating two sequences, or finding the size,

making a sequence with all elements the same

making a sequence into a tuple or set (rng also makes it into a set),

testing for empty, prefix, or sub-sequence (not necessarily contiguous),

lexical comparison, permuting, and sorting,

treating a sequence as a multiset with operations to:

count the number of times an element appears, test membership and multiset equality,

take differences, and remove an element $("+"$ or $" \backslash /$ " is union and addl adds an

element).

All these operations are undefined if they use out-of-range subscripts, except that an empty subsequence is defined regardless of the subscripts.

We define the sequence methods with a module. Precisely, SEQ T is Sequence $[T] \cdot Q$, where:

\section{MODULE Sequence [T] EXPORTS $Q=$}

TYPE I

$=(\mathrm{I} \rightarrow \mathrm{T})$

$\operatorname{SUCHTHAT}(\backslash \mathrm{q} \mid(\operatorname{ALL} i \mid \mathrm{q} ! \mathrm{i}=(0<=i 八 i<\mathrm{q}$. size $)))$

WITH $\{$ size:=Size, sub:=Sub, $"+":=$ Concatenate,

head:=Head, tail:=Tail, addh:=AddHead, remh:=Tail,

last:=Last, reml:=Removelast, addl $:=$ AddLast

seg:=Seg, fill:=Fill, tuple: $=$ Tuple,

isEmpty:=IsEmpty, $"<=":=$ Prefix, $"<<=":=$ SubSeq,

lexLE:=LexLE, perms:=Perms,

fsorter:=FSorter, fsort:=FSort, sort:=Sort,

$\%$ These methods treat a sequence as a multiset (or bag). count:=Count, "IN":=In, $"==":=$ EqElem, " \/":=Concatenate, " $"$ :=Diff, set:=Q.rng

FUNC Size(q) $->$ Int $=$ RET q.dom.size

FUNC $\operatorname{Sub}(q, i 1, i 2)->Q=$

FUNC Concatenate $(q 1, q 2) \rightarrow Q=\operatorname{VAR} q \mid \quad \% q 1+q 2$

q. $\operatorname{sub}(0, q 1 . s i z e-1)=q 1 / q . \operatorname{sub}(q 1 . \operatorname{size}, q \cdot \operatorname{size}-1)=q 2 \Rightarrow \operatorname{RET} q$

FUNC Head $(q) \rightarrow T=$ RET $q(0)$

FUNC Tail (q) $\rightarrow Q=$

q.size $>0 \Rightarrow \operatorname{RET} q . \operatorname{sub}(1, q . \operatorname{size}-1)$

FUNC AddHead $(q, t) \rightarrow Q=\operatorname{RET}\{t\}+q$
$\% \mathrm{q}$. head; first element

$\%$ q.addh ( $\mathrm{t}$ )
$\%$ q.tail; all but first 
FUNC Last $(q) \rightarrow T=\operatorname{RET} q(q . s i z e-1)$

FUNC RemoveLast $(q) \rightarrow Q=$

q \# \{\}$\Rightarrow \operatorname{RET}$ q.sub $(0, q . \operatorname{size}-2)$

FUNC AddLast $(q, t) \rightarrow Q=\operatorname{RET} q+\{t$

$\%$ q. last; last element

$\%$ q.reml; all but last

$\% \mathrm{q} \cdot \operatorname{addl}(t)$

FUNC Seg(q, $i, n: I) \rightarrow Q=\operatorname{RET} q . \operatorname{sub}(i, i+n-1)$

$\%$ q. seg $(i, n) ; n$ T's from q(i)

FUNC Fill $(t, n: I) \rightarrow Q=\operatorname{RET}\{i: I N \quad 0 \ldots n-1|| t\} \quad \%$ yields $i$ copies of $t$

FUNC IsEmpty $(q) \rightarrow$ Bool $=\operatorname{RET}(q=\{\})$

FUNC Prefix(q1, q2) $\rightarrow$ Bool $=$

$$
\operatorname{RET}(\operatorname{EXISTS} q \mid \mathrm{q} 1+\mathrm{q}=\mathrm{q} 2 \text { ) }
$$

$\% \mathrm{q} 1<=\mathrm{q} 2$

FUNC SubSeq(q1, q2) $\rightarrow$ Bool $=$

$\%$ Are $\mathrm{q} 1$ 's elements in $\mathrm{q} 2$ in the same order, not necessarily contiguously.

$$
\text { RET (EXISTS p: SET Int } \mid p<=q 2 \text {.dom } \wedge \text { } q 1=\text { p. seq. sort * } \mathrm{q}^{2} \text { ) }
$$

FUNC LexLE (q1, q2, $\mathrm{f}:(\mathrm{T}, \mathrm{T})->\mathrm{Bool}) \rightarrow$ Bool $=$

$\% \mathrm{q} 1 . \operatorname{lexLE}(\mathrm{q} 2, \mathrm{f}) ; \mathrm{f}$ is $<=$

$\%$ Is $q 1$ lexically less than or equal to $q 2$. True if $q 1$ is a prefix of $q 2$,

$\%$ or the first element in which $\mathrm{q} 1$ differs from $\mathrm{q} 2$ is less.

$$
\text { RET } \mathrm{q} 1<=q 2
$$

$\backslash\left(\operatorname{EXISTS} i:\right.$ IN $q 1 . \operatorname{dom} 八 \mathrm{q}^{2} . \operatorname{dom} \mid q 1 \cdot \operatorname{sub}(0, i-1)=q 2 . \operatorname{sub}(0, i-1)$

$$
\text { 八 q1(i) \#q2(i)) 八f(q1(i), q2(i)) }
$$

FUNC Perms $(q)->$ SET $Q=$

$\operatorname{RET}\left\{q^{\prime} \mid\left(\operatorname{ALL} t \mid q \cdot \operatorname{count}(t)=q^{\prime} \cdot \operatorname{count}(t)\right)\right\}$

FUNC FSorter $(q, f:(T, T)->$ Bool) $)->$ SEQ Int $=\quad$ \% q.fsorter $(f) ; f$ is $<=$

$\%$ The permutation that sorts q stably. Note: can't use min to define this, since min is defined using sort.

VAR ps: $:\{$ : IN q.dom. seq.perms $\quad \%$ all perms that sort $q$

VAR p0 :IN ps |

$$
\text { (ALL } p: \text { IN ps } \mid \text { po.lexLE (p, Int." }<=")) \Rightarrow \operatorname{RET} \text { p0 }
$$

FUNC FSort (q, f: $(\mathrm{T}, \mathrm{T})->$ Bool) $\rightarrow \mathrm{Q}=\quad$ \% q.fsort $(\mathrm{f}) ; \mathrm{f}$ is $<=$ for the sort RET $q$. fsorter $(f) * q$

FUNC Sort $(\mathrm{q})->Q=$ RET q.fsort $(\mathrm{T} . "<=") \quad$ \% q. sort; only if $\mathrm{T}$ has $<=$

FUNC Count $(q, t)->$ Int $=\operatorname{RET}\left\{t^{\prime}:\right.$ IN $\left.q \mid t^{\prime}=t\right\}$.size \%q.count $(t)$

FUNC In $(t, q)->$ Bool $=\operatorname{RET}(q \cdot \operatorname{count}(t) \# 0) \quad \% t$ IN $q$

FUNC EqElem(q1, q2) $\rightarrow$ Bool = RET q1 IN q2.perms $\quad \%$ q1 == q2; equal as multisets

FUNC Diff(q1, q2) $\rightarrow Q=$

$\% \mathrm{q} 1-\mathrm{q} 2$

$\operatorname{RET}\{q \mid(\operatorname{ALL} t \mid q \cdot \operatorname{count}(t)=\{q 1 \cdot \operatorname{count}(t)-q 2 \cdot \operatorname{count}(t), 0\} \cdot \max )\} \cdot$ choose

END Sequence

We can't program Tuple in Spec, but it is defined as follows. If $q$ : $S E Q T$, then q.tuple is a tuple of q.size T's, the first equal to $q(0)$, the second equal to $q(1)$, and so forth. For the inverse, if $u$ is a tuple of T's, then $u$. seq is a SEQ T such that $u$.seq.tuple $=u$. If $u$ is a tuple in which not all the elements have the same declared type, then u. seq is a SEQ Any such that u. seq.tuple $=u$.

Int has a method $\ldots$ for making sequences: $i \ldots j=\{i, i+1, \ldots, j-1, j\}$. If $j<i$, $i \ldots j=\{\}$. You can also write $i \ldots j$ as $\{k:=i$ BY $k+1$ WHILE $k<=j\}$; see [11] in section 5. Int also has a seq method: $i$.seq $=0 \ldots i-1$.
There is a constructor $\{e 1, e 2, \ldots\}$ for a sequence with specific elements and a constructor \{\} for the empty sequence. There is also a constructor $q\{e 1->e 2\}$, which is equal to $q$ except at $e 1$ (and undefined if e1 is out of range). For the constructors see [6] and [8] of section 5. To generate a sequence there are constructors $\{x: I N q \mid$ pred $\mid \exp \}$ and $\{x:=$ e1 BY e2 WHILE pred1 | pred2 | exp\}. For these see [11] of section 5.

To map each element $t$ of $q$ to $f(t)$ use function composition $q * f$. Thus if $q$ : SEQ Int, $q *(\backslash i$ : Int $\mid i * i)$ yields a sequence of squares. You can also write this $\{i: I N q|| i * i\}$. 


\section{Index}

$-, 9,19,21$

!, 9,22

$! !, 9,22$

$\#, 9,10$
$\div, 3$

( ), $3,8,16$

(expList), 8

(typelist), 5

$\star *, 9$

$, 3,5$

/, 9

$/, 9$

$\wedge, 9,20$
$:, 8,14$

$, 3,5$

$=, 3,8,14,18$

$;, 14$

[ ] $, 3,14$

[declList]

[n], 3

, 8

$\backslash /, 9,20$

$\{* \quad \rightarrow$ result $\}, 8$

\{\}$, 3,8$

\{decllist | pred | exp\}, 8

\{exceptionList\}, 5

$\{$ exp $\rightarrow$ result $\}, 8$

\{expList\}, 8

\{methodDefList\}, 5, 6

\{\}, 21

$\{e 1, e 2, \ldots\}, 21$

$\sim, 9$

$+, 5,9,19,21,22$

$>, 9$

$</<<, 14,16$

$<<>>, 3$

$<<=, 9,22$

$<=, 19,21$

$=, 9,10$

$==>, 9$

$=>, 3,8,14$

$>, 9$

$<=/>, 9$

$>>14$

abstract equality, 10

add, 9

add an element, 9 adding an element, 19, 22

addl, 22

ALL, 8

Any, 5, 10

append an element, 9

APROC, 5, 16

arguments, 8

AS, 8

assignment, 14

associative, 6, 10, 14

atomic command, 1, 13, 14

atomic procedure, 2

backtracking, 13 expanded definitions, 4

bag, $22 \quad$ expression, 1,7

BEGIN, 14

body, 16

Bool, 5

built-in methods, 19

capital letter, 3

Char, 5

characteristic predicate, 19

choice, 14

choosing an element, 19

CLASS, 16

class, 17
closure, 21

closure, 21

cmd, 14

command, 1,13

comment, 3

composition, 20

concatenation, 9

conditional, 10

conditional and, 9

conditional or,

CONST, 17

constructor, 8

count, 22

data abstraction, 6

decl, 5

declaration, 18

defined, 9,20

difference, 22

divide, 9

DO, 14

dot, 18

e.id, 10

e.id(), 10

e1 infixOp e2, 10 e1.id (e2), 10

else, 14

$\mathrm{y}, 3,10$

mpty sequence, 22

empty set, 19

ENUM, 16

equal, 9

EXCEPT, 14

exception, 5, 6, 7, 15, 16

tionset, 5, 16

expression has a type, 7

FI, 14

fill, 22

fit, $4,7,11,14,15$

formal parameters, 16

function, 2, 6, 14, 19, 20

function undefined everywhere, 20

general procedure, 2

Global.id, 16, 18

grammar, 3

graph, 21

greater or equal, 9

greater than, 9

grouping, 15

guard, 13,14

guard, 13, 14

has a routine type, 4

has type $\mathrm{T}, 4$

Havoc, 14

head, 22

id, 3,6

id := exp, 8

id [ typelist ],5

identifier, 3

if, 14

implies, 9

N, 9, 19, 22

includes, 4

initial value, 17

nitialize, 15

instantiate, 16

intersection, 9, 19

Introduction to Spec, 1 invocation, 7, 8, 10, 14

IS, 8

isEmpty, 22

isPath, 21

known, 20

LAMBDA, 8, 11

last, 19

lexical comparison, 22

lexical co

List, 3
literal, 3,7 ,

local, 18

ooping exception, 7,13

m[typeList] .id, 6

$\max , 19$

meaning

of an atomic command, 13

of an expression, 7

membership, 9,19

method, 4, 5, 6, 19

mfp, 16

min, 19

module, 2, 16

multiply, 9

multiset, 22

multiset difference, 9

name, $1,5,18$

new variable, 15

non-atomic command, 2, 13

Non-Atomic Semantics of Spec, 1

non-deterministic, 1

nonterminal symbol, 3

normal result, 20

not equal, 9

Null, 5

OD, 14

operator, 3,6

Orderedset, 19

organizing your program, 2

outcome, 13

parameterized module, 16

path in the graph, 2

precedence, $6,9,14$

precondition, 14
pred, 8,19

predefined identifiers, 3

predicate, 20

prefix, 9,22

prefixop, 9

prefixop e, 10

primary, 8
PROC, 5,16

program, 2,16

punctuation, 3

quantif, 8

quantification, 10

RAISE, 8, 14

RAISE exception, 11

RAISES, 5, 11

RAISES set, 15

record, 5,10

redeclaration, 18

relation, 21

lement, 9, 19, 22

result, 7

result type, 14

RET, 14

routine, $2,14,16$

scope, 18

SEQ, 5, 6, 22

SEQ Char, 6

sequence, 22

sequential composition, 14

sequential program, 2

SET, 5, 10, 11, 19, 20

set difference, 9,19

set of sequences of states, 2

set of values, 4

et with specific elements, 19

setF, 2

side effects, 15

signature, 15, 16

SKIP, 14

specifications, 1

state, $1,7,13,18$

state variable, 1

String, 5, 6

stringliteral, 5

sub-sequence, 9,22

subset, 9,19

subtract, 9

symbol, 3

syntactic sugar, 7

T. m, 6, 7

T->U, 6

tail, 22

terminal symbol, 3

test membership, 19, 22

thread, 17

toplevel, 16, 18 transition, 1

transitive closure, 2

tuple, 5, 14, 15

tuple constructor, 8

type, 2, 4, 5, 16

type equality, 4

type-checking, 4, 7, 14

undefined $7,10,13$

UNION, 5, 6, 7, 9, 19, 22

upper case, 3

value,

variable, $1,14,15$

white space, 3

WITH, 5, 6, 10, 16 


\section{Examples of Specifications and Implementations}

This handout is a supplement for the first two lectures. It contains several example specifications and implementations, all written using Spec.

Section 1 contains a specification for sorting a sequence. Section 2 contains two specifications and one implementation for searching for an element in a sequence. Section 3 contains specifications for a read/write memory. Sections 4 and 5 contain implementations for a $\mathrm{read} /$ write memory based on caching and hashing, respectively. Finally, Section 6 contains an implementation based on replicated copies.

\section{Sorting}

The following specification describes the behavior required of a program that sorts sets of some type $\mathrm{T}$ with a "<=" comparison method. We do not assume that $"<=$ " is antisymmetric; in other words, we can have $t 1<=t 2$ and $t 2<=t 1$ without having $t 1=t 2$, so that " $<="$ is not enough to distinguish values of $\mathrm{T}$. For instance, $\mathrm{T}$ might be the record type [name:String, salary: Int] with "<=" comparison of the salary field. Several T's can have different names but the same salary.

APROC Sort (S: SET T) $\rightarrow$ SEQ $T=<<$

VAR $q: \operatorname{SEQ} T \mid(\operatorname{ALL} i: T \mid s \cdot \operatorname{count}(i)=q \cdot \operatorname{count}(i)) /$ Sorted (b) $\Rightarrow \operatorname{RET} b>>$

This specification uses the auxiliary function sorted, defined as follows.

FUNC Sorted(q: SEQ T) $\rightarrow$ Bool $=\operatorname{RET}($ ALL $i$ : IN q.dom $-\{0\} \mid q(i-1)<=q(i)$ )

If we made Sort a FUNC rather than a PROC, what would be wrong? ${ }^{1}$ What could we change to make it a FUNC?

We could have written this more concisely as

APROC SOrt (S: SET T) $\rightarrow$ SEQ $T=$

$$
<\text { VAR q : IN a.perms | Sorted }(q) \Rightarrow \text { RET q >> }
$$

using the perms method for sets that returns a set of sequences that contains all the possible permutations of the set.

\section{Searching}

\section{Search specification}

We begin with a specification for a procedure to search an array for a given element. Again, this is an APROC rather than a FUNC because there can be several allowable results for the same inputs.

\footnotetext{
${ }^{1}$ Hint: a FUNC can't have side effects and must be deterministic (return the same value for the same arguments).
}

APROC Search (q: SEQ T, $x: T$ ) $\rightarrow$ Int RAISES $\{$ NotFound $\}=$

< IF VAR i: Int $\mid(0<=i 八 i<q$. size $八 \mathrm{q}(\mathrm{i})=\mathrm{x}) \Rightarrow \operatorname{RET} i$

[*] RAISE NotFound

FI $>>$

Or, equivalently but slightly more concisely:

APROC Search (q: SEQ T, $x$ : T) $\rightarrow$ Int RAISES $\{$ NotFound $\}=$

$<$ IF VAR $i$ :IN q.dom $\mid q(i)=x=$ RET $i\left[{ }^{*}\right]$ RAISE NotFound FI $>$

Sequential search implementation

Here is an implementation of the search specification given above. It uses sequential search, starting at the first element of the input sequence.

APROC SeqSearch (q: SEQ T, $\mathrm{x}: \mathrm{T})->$ Int RAISES $\{$ NotFound $\}=<$ VAR $i:=0$

DO $i<q . s i z e=>$ IF $q(i)=x \Rightarrow$ RET $i\left[{ }^{*}\right] i+:=1$ FI OD; RAISE NotFound $>>$

Alternative search specification

Some searching algorithms, for example, binary search, assume that the input argument sequence is sorted. Such algorithms require a different specification, one that expresses this requirement.

APROC Search1 (q: SEQ T, $x: T$ ) $\rightarrow$ Int RAISES $\{$ NotFound $\}=<<$

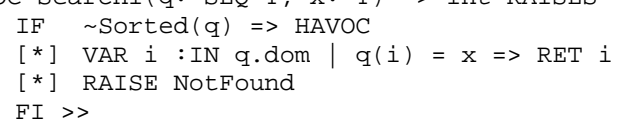

$\mathrm{FI}>>$

You might consider writing the specification to raise an exception when the array is not sorted:

APROC Search2 (q: SEQ T, $x: T$ ) $\rightarrow$ Int RAISES $\{$ NotFound, NotSorted $\}=<<$

IF $\sim$ Sorted $(q) \Rightarrow$ RAISE NotSorted$$
\text { ... }
$$

This is not a good idea. The whole point of binary search is to obtain $\mathrm{O}(\log n)$ time performance (for a sorted input sequence). But any implementation of the Search2 specification requires an $\mathrm{O}(n)$ check, even for a sorted input sequence, in order to verify that the input sequence is in fact sorted.

This is a simple but instructive example of the difference between defensive programming and efficiency. If search were part of an operating system interface, it would be intolerable to have HAVOC as a possible transition, because the operating system is not supposed to go off the deep end no matter how it is called (though it might be OK to return the wrong answer if the input isn't sorted; what would that spec be?). On the other hand, the efficiency of a program often depends on assumptions that one part of it makes about another, and it's appropriate to express such an assumption in a spec by saying that you get HAVOC if it is violated. We don't care to be more specific about what happens because we intend to ensure that it doesn't happen. Obviously a program written in this style will be more prone to undetected or obscure errors than one that checks the assumptions, as well as more efficient. 


\section{Read/write memory}

The simplest form of read/write memory is a single read/write register, say of type D (for data), with arbitrary initial value. The following Spec module describes this:

\section{MODULE Register [D] EXPORT Read, Write =}

VAR $x: D$

$\%$ arbitrary initial value

APROC Read() $\rightarrow D=\langle<\operatorname{RET} x\rangle$

APROC Write (d) $=\langle<\mathrm{x}:=\mathrm{d}\rangle\rangle$

END Register

Now we give a specification for a simple addressable memory with elements of type D. This is like a collection of read/write registers, one for each address in a set A. In other words, it's a

function from addresses to data values. For variety, we include new Reset and Swap operations in addition to Read and write.

MODULE Memory [A, D] EXPORT Read, Write, Reset, Swap =

TYPE M $=$ A $\rightarrow D$

$\operatorname{VAR} m:=\operatorname{Init}()$

APROC Init () $->M=\left\langle\right.$ VAR $\left.m^{\prime}\right|$ (ALL a $\left.\mid m^{\prime} ! a\right)=>$ RET $m^{\prime}>>$

$\%$ Choose an arbitrary function that is defined everywhere.

FUNC Read(a) $\rightarrow$ D $=\langle<\operatorname{RET} m(a)>>$

$\operatorname{APROC} \operatorname{Write}(a, d)=\langle<m(a):=d>>$

APROC Reset $(\mathrm{d}) \quad=\left\langle<\mathrm{m}:=\mathrm{M}\left\{{ }^{*}->\mathrm{d}\right\}>>\right.$

$\%$ Set all memory locations to $d$.

APROC Swap $(a, d) \rightarrow D=\left\langle\operatorname{VAR} d^{\prime}:=m(a)\right| m(a):=d ; \operatorname{RET} d^{\prime}>>$

$\%$ Set location a to the input value and return the previous value.

END Memory

The next three sections describe implementations of Memory.

\section{Write-back cache implementation}

Our first implementation is based on two memory mappings, a main memory $\mathrm{m}$ and a write-back cache $\mathrm{c}$. The implementation maintains the invariant that the number of addresses at which $\mathrm{c}$ is defined is constant. A real cache would probably maintain a weaker invariant, perhaps bounding the number of addresses at which $\mathrm{c}$ is defined.

MODULE WBCache $[\mathrm{A}, \mathrm{D}]$ EXPORT Read, Write, Reset, Swap = $\%$ implements Memory

$\begin{array}{ll}\operatorname{TYPEM} & =\mathrm{A} \rightarrow \mathrm{D} \\ \mathrm{C} & =\mathrm{A} \rightarrow \mathrm{D} \\ \text { CONST Csize } & : \text { Int }:=\ldots \\ \operatorname{VAR} \mathrm{m} & :=\operatorname{InitM}() \\ \mathrm{C} & :=\operatorname{InitC}()\end{array}$

$\%$ cache size

APROC Initm() $\rightarrow M=<$ VAR $m^{\prime} \mid$ (ALL a $\left.\mid m^{\prime} ! a\right) \quad \Rightarrow$ RET $m^{\prime}>$ $\%$ Returns a $\mathrm{M}$ with arbitrary values.

APROC InitC () $\rightarrow C=\left\langle<\operatorname{VAR} C^{\prime}\right| C^{\prime}$.dom.size = CSize $\Rightarrow$ RET $\left.C^{\prime}\right\rangle>$ $\%$ Returns a $\mathrm{C}$ that has exactly CSize entries defined, with arbitrary values.

APROC Read(a) $\rightarrow D=<<\operatorname{Load}(a) ; \operatorname{RET} C(a)>>$

APROC Write $(a, d)=\langle<$ IF $\sim C$ ! a $\Rightarrow$ Flushone () [*] SKIP FI; $c(a):=d>>$ $\%$ Makes room in the cache if necessary, then writes to the cache.

APROC Reset $(d)=\langle<\ldots\rangle\rangle$

APROC $\operatorname{Swap}(\mathrm{a}, \mathrm{d}) \rightarrow \mathrm{D}=\left\langle<\operatorname{VAR} d^{\prime}\right| \operatorname{Load}(\mathrm{a}) ; \mathrm{d}^{\prime}:=\mathrm{C}(\mathrm{a}) ; \mathrm{c}(\mathrm{a}):=\mathrm{d} ; \operatorname{RET} \mathrm{d}^{\prime}>>$

$\%$ Internal procedures.

APROC Load $(a)=\langle<$ IF $\sim C ! a \Rightarrow$ Flushone ()$; C(a):=m(a)[*]$ SKIP FI $>>$ $\%$ Ensures that address a appears in the cache.

APROC Flushone () =

$\%$ Removes one (arbitrary) address from the cache, writing the data value back to main memory if necessary. $<<\operatorname{VAR} a \mid c ! a=\operatorname{IF} \operatorname{Dirty}(\mathrm{a}) \Rightarrow \mathrm{m}(\mathrm{a}):=\mathrm{c}(\mathrm{a})\left[{ }^{*}\right] \operatorname{SKIP} \mathrm{FI} ; \mathrm{c}:=\mathrm{c}\{\mathrm{a} \rightarrow>\}>$ FUNC Dirty (a) $\rightarrow$ Bool $=$ RET $c ! a 八 c(a) \# m(a)$

$\%$ Returns true if the cache is more up-to-date than the main memory.

END WBCache

The following Spec function is an abstraction function mapping a state of the wBCache module to a state of the Memory module. It's written to live inside the module. It says that the contents of location $a$ is $c(a)$ if $a$ is in the cache, and $m(a)$ otherwise.

FUNC AF() $\rightarrow M=\operatorname{RET}(\backslash a \mid c ! a \Rightarrow c(a) \quad[*] m(a)$ 


\section{Hash table implementation}

Our second implementation of Memory uses a hash table for the representation.

MODULE HashMemory [A WITH $\{$ hf: A->Int $\}$, D] EXPORT Read, Write, Reset, Swap = $\%$ Implements Memory.

$\%$ The module expects that the hash function $\mathrm{A} . \mathrm{hf}$ is total and that its range is $0 \ldots \mathrm{n}$ for some $\mathrm{n}$.

TYPE Pair $\quad=[a, d]$
$\begin{array}{ll}\mathrm{B} & =\mathrm{SEQ} \text { Pair } \\ \text { HashT } & =\mathrm{SEQ} \mathrm{B}\end{array}$

$\operatorname{VAR} \mathrm{nb} \quad:=\operatorname{NumB}()$

$\mathrm{m} \quad:=$ HashT.fill $(\mathrm{B}\{\}, \mathrm{nb}) \quad \%$ Memory hash table; initially empty

ROC Read (a) $\rightarrow D=<\operatorname{VAR} b:=m(a . h f), i:$ Int |

$i:=$ FindEntry $(a, b)$ EXCEPT NotFound $=>$ RET default ; RET b(i).d >>

$\operatorname{APROC} \operatorname{Write}(a, d)=<\operatorname{VAR} b:=\operatorname{DeleteEntry}(\mathrm{a}, \mathrm{m}(\mathrm{a} . \mathrm{hf}))$

$m(a . h f):=b+\{$ Pair $\{a, d\}\}>>$

APROC Reset $(\mathrm{d})=\langle<\mathrm{m}:=\operatorname{HashT}$.fill $(\mathrm{B}\{\}, \mathrm{nb}) ;$ default $:=\mathrm{d}>>$

APROC Swap (a, d) $\rightarrow D=\left\langle\operatorname{VAR} d^{\prime}\right| d^{\prime}:=\operatorname{Read}(a) ; \operatorname{Write}(a, d) ; \operatorname{RET} d^{\prime}>>$

\section{\% Internal procedures.}

FUNC NumBs () $\rightarrow$ Int $=$

$\%$ Returns the number of buckets needed by the hash function; havoc if the hash function is not as expected

IF VAR $n$ : Nat A.hf.rng $=(0 \ldots \mathrm{n})$. set $\Rightarrow$ RET $n+1\left[{ }^{*}\right]$ HAVOC FI

APROC Findentry $(a, b) \rightarrow$ Int RAISES (NotFound) $=$

$\%$ If a appears in a pair in $b$, returns the index of some pair containing $a$; otherwise raises NotFound.

$<$ VAR i : IN b.dom $\mid \mathrm{b}(\mathrm{i}) \cdot \mathrm{a}=\mathrm{a}=>$ RET i [*] RAISE NotFound $>>$

APROC DeleteEntry $(a, b) \rightarrow B<\operatorname{VAR} i$ : Int

$\%$ Removes some pair with address a from $b$, if any exists.

$i:=$ Findentry $(a, b)$ EXCEPT NotFound $\Rightarrow$ RET $b$;

RET b.sub(0, i-1) + b.sub(i+1, b.size-1) >>

END HashMemory

Note that FindEntry and DeleteEntry are APROCs because they are not deterministic when given arbitrary $\mathrm{b}$ arguments.

The following is a key invariant that holds between invocations of the operations of HashMemory:

FUNC Inv () $\rightarrow$ BOol $=$ RET

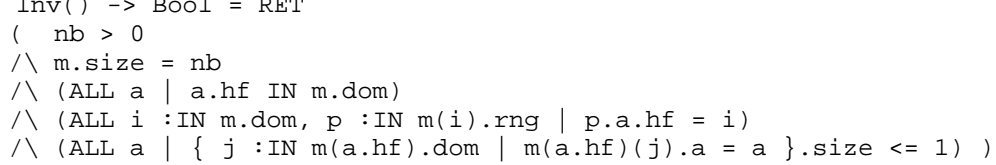

This says that the number of buckets is positive, that the hash function maps all addresses to actual buckets, that a pair containing address a appears only in the bucket at index a.hf in $\mathrm{m}$, and that at most one pair for an address appears in the bucket for that address. Note that these conditions imply that in any reachable state of HashMemory, each address appears in at most one pair in the entire memory.

The following Spec function is an abstraction function between states of the HashMemory module and states of the Memory module:

FUNC AF() $\rightarrow M=$ RET

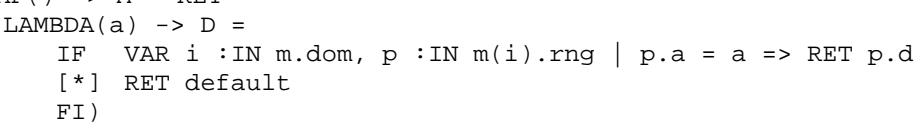

That is, the data value for address a is any value associated with address a in the hash table; if there is none, the data value is the default value. Spec says that a function is undefined at an argument if its body can yield more than one result value. The invariants given above ensure that the LAMBDA is actually single-valued for all the reachable states of HashMemory.

Of course HashMemory is not a fully detailed implementation. Its main deficiency is that it doesn't explain how to maintain the variable-length bucket sequences, which is usually done with a linked list. However, the implementation does capture all the essential details.

\section{Replicated copies}

Our final implementation is based on some number $k \geq 1$ of copies of each memory location Initially, all copies have the same default value. A write operation only modifies an arbitrary majority of the copies. A Read reads an arbitrary majority, and selects and returns the most recent of the values it sees. In order to allow the Read to determine which value is the most recent, each write records not only its value, but also a sequence number.

For simplicity, we just show the module for a single read/write register. The constant $\mathrm{k}$ determines the number of copies.

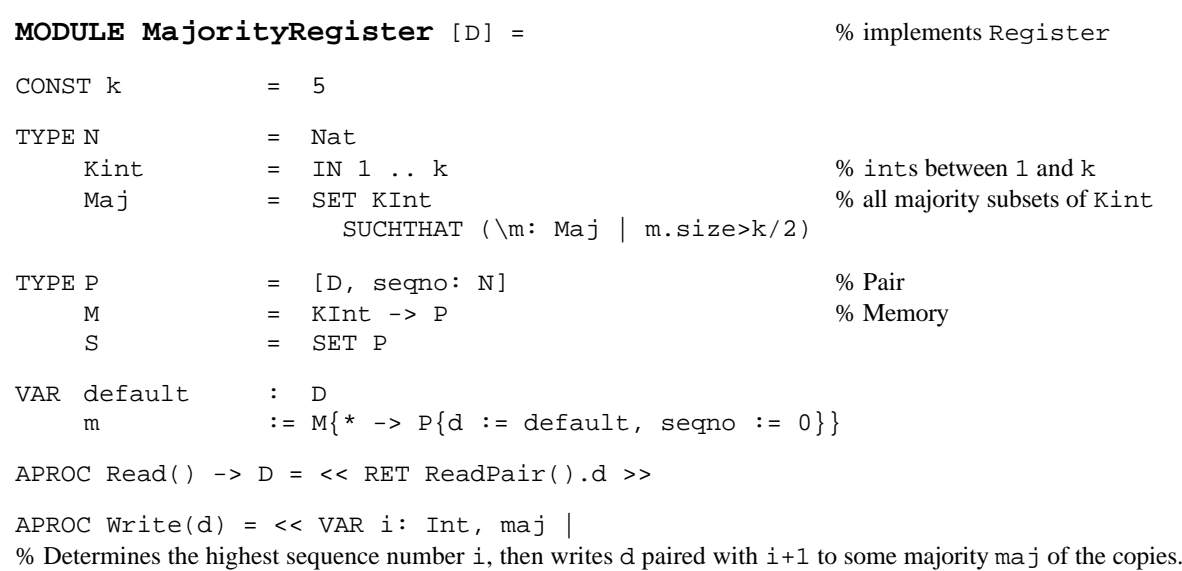


i : = ReadPair (). seqno;

DO VAR $j: \operatorname{IN} m a j \mid m(j)$.seqno \# $i+1=m(j):=P\{d:=d$, seqno := $i+1\}$ OD $>>$

\section{$\%$ Internal procedures.}

APROC ReadPair() $\rightarrow P=<$ VAR $s:=$ ReadMaj () |

$\%$ Returns a pair with the largest sequence number from some majority of the copies.

VAR $\mathrm{p}:$ IN $\mathrm{s} \mid \mathrm{p}$.seqno $=\left\{\mathrm{p}^{\prime}:\right.$ IN $\mathrm{s}|| \mathrm{p}^{\prime} \cdot$ seqno $\}$ max $\Rightarrow$ RET $\mathrm{p} \gg$

APROC ReadMaj () $\rightarrow S=\langle<\operatorname{VAR} \operatorname{maj}| \operatorname{RET}\{i: I N \operatorname{maj}|| m(i)\}>>$

$\%$ Returns the set of pairs belonging to some majority of the copies.

END MajorityRegister

We could have written the body of ReadPair as

<< VAR s := ReadMaj() | RET s.fmax((\p1, p2 $\mid$ p1.seqno <= p2.seqno)) >> except that fmax always returns the same maximal $p$ from the same $s$, whereas the VAR in ReadPair chooses one non-deterministically.

The following is a key invariant for MajorityRegister.

FUNC Inv (m: M) $\rightarrow$ BOOI $=$ RET

(ALL $p$ : IN m.rng, $p^{\prime}$ : IN $m$.rng $\mid p$. seqno $=p^{\prime}$. seqno $==>p \cdot d=p^{\prime} \cdot d$ )

The first conjunct says that any two pairs having the same sequence number also have the same data. The second conjunct says that the highest sequence number appears in some majority of the copies.

The following Spec function is an abstraction function between states of the MajorityRegister module and states of the Register module.

FUNC $A F() \rightarrow D=R E T m . r n g . f m a x((\backslash p 1, p 2 \mid p 1$.seqno <=p2.seqno $)) \cdot d$

That is, the abstract register data value is the data component of a copy with the highest sequence number. Again, because of the invariants, there is only one $p . d$ that will be returned. 


\section{Abstraction Functions and Invariants}

This handout describes the main techniques used to prove correctness of state machines: abstraction functions and invariant assertions. We demonstrate the use of these techniques for some of the Memory examples from handout 5.

Throughout this handout, we consider modules all of whose externally invocable procedures are APROCS. We assume that the body of each such procedure is executed all at once. Also, we do not consider procedures that modify global variables declared outside the module under consideration.

\section{Modules as state machines}

Our methods apply to an arbitrary state machine or automaton. In this course, however, we use a Spec module to define a state machine. Each state of the automaton designates values for all the variables declared in the module. The initial states of the automaton consist of initial values assigned to all the module's variables by the Spec code. The transitions of the automaton correspond to the invocations of APROCS together with their result values.

An execution fragment of a state machine is a sequence of the form $s_{0}, \pi_{1}, s_{1}, \pi_{2}, \ldots$, where each $s$ is a state, each $\pi$ is a label for a transition (an invocation of a procedure), and each consecutive $\left(s_{i}, \pi_{i+1}, s_{i+1}\right)$ triple follows the rules specified by the Spec code. (We do not define these rules here-wait for the lecture on atomic semantics.) An execution is an execution fragment that begins in an initial state.

The $\pi_{i}$ are labels for the transitions; we often call them actions. When the state machine is written in Spec, each transition is generated by some atomic command, and we can use some unambiguous identification of the command for the action. At the moment we are studying sequential Spec, in which every transition is the invocation of an exported atomic procedure. We use the name of the procedure, the arguments, and the results as the label.

Figure 1 shows some of the states and transitions of the state machine for the Memory module with $\mathrm{A}=\mathrm{IN} 1 \ldots 4$, and Figure 2 does likewise for the $\mathrm{WBCache}$ module with Csize $=2$. The arrow for each transition is labeled by its $\pi_{i}$, that is, by the procedure name, arguments, and result.

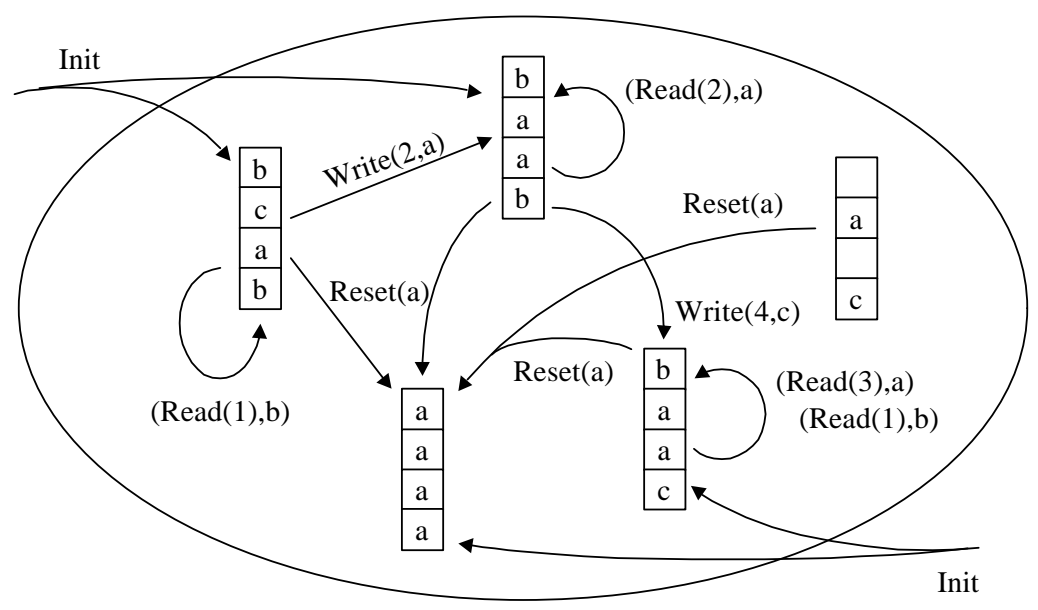

Figure 1: Part of the Memory state machine

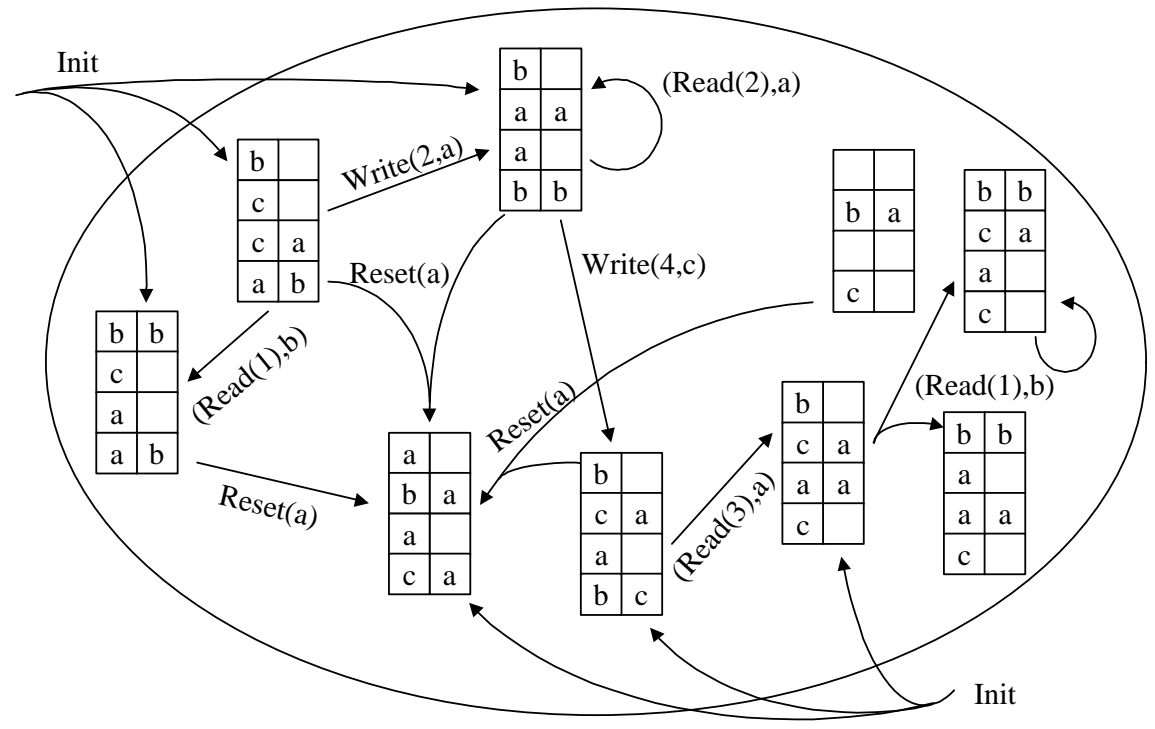

Figure 2: Part of the WBCache state machine 


\section{External behavior}

Usually, a client of a module is not interested in all aspects of its execution, but only in some kind of external behavior. Here, we formalize the external behavior as a set of traces. That is, from an execution (or execution fragment) of a module, we discard both the states and the internal actions, and extract the trace. This is the sequence of labels $\pi_{i}$ for external actions (that is, invocations of exported routines) that occur in the execution (or fragment). Then the external behavior of the module is the set of traces that are obtained from all of its executions.

It's important to realize that in going from the execution to the trace we are discarding a great deal of information. First, we discard all the states, keeping only the actions or labels. Second, we discard all the internal actions, keeping only the external ones. Thus the only information we keep in the trace is the behavior of the state machine at its external interface. This is appropriate, since we want to study state machines that have the same behavior at the external interface; we shall see shortly exactly what we main by 'the same' here. Two machines can have the same traces even though they have very different state spaces.

In the sequential Spec that we are studying now, a module only makes a transition when an exported routine is invoked, so all the transitions appear in the trace. Later, however, we will introduce modules with internal transitions, and then the distinction between the executions and the external behavior will be important.

For example, the set of traces generated by the Memory module includes the following trace:

$$
\begin{aligned}
& \text { (Reset (d), ) } \\
& \text { (Read (a1), d) } \\
& \text { (Write (a2, d')) } \\
& (\text { Read (a2), d') }
\end{aligned}
$$

However, the following trace is not included if $d^{\#} d^{\prime}$ :

$$
\begin{aligned}
& \text { (Reset (d)) } \\
& \text { (Read (a1), } d^{\prime} \text { ) } \\
& \text { (Write (a2, } d^{\prime} \text { ) }
\end{aligned}
$$$$
(\operatorname{Read}(a 2), d)
$$

In general, a trace is included in the external behavior of Memory if every Read (a) or Swap (a, d) operation returns the last value written to a by a write, Reset or Swap operation, or returned by a Read operation; if there is no such previous operation, then $\operatorname{Read}(a) \operatorname{or} \operatorname{Swap}(a, d)$ returns an arbitrary value.

\section{Implements relation}

In order to understand what it means for one state machine to implement another one, it is helpful to begin by considering what it means for one atomic procedure to implement another. The meaning of an atomic procedure is a relation between an initial state just before the procedure starts (sometimes called a 'pre-state') and a final state just after the procedure has finished (sometimes called a 'post-state'). This is often called an 'input-output relation'. For example, the relation defined by a square-root procedure is that the post-state is the same as the pre-state, except that the square of the procedure result is close enough to the argument. This meaning makes sense for an arbitrary atomic procedure, not just for one in a trace.
We say that procedure $P$ implements spec $S$ if the relation defined by $P$ (considered as a set of ordered pairs of states) is a subset of the relation defined by $S$. This means that $P$ never does anything that S couldn't do. However, $\mathrm{P}$ doesn't have to do everything that $\mathrm{S}$ can do. An implementation of square root is probably deterministic and always returns the same result for a given argument. Even though the spec allows several results (all the ones that are within the specified tolerance), we don't require an implementation to be able to produce all of them; instead we are satisfied with one.

Actually this is not enough. The definition we have given allows $P$ 's relation to be empty, that is, it allows $P$ not to terminate. This is usually called 'partial correctness'. In addition, we usually want to require that $P$ 's relation be total on the domain of $S$; that is, $P$ must produce some result whenever $S$ does. The combination of partial correctness and termination is usually called 'total correctness'.

If we are only interested in external behavior of a procedure that is part of a stateless module, the only state we care about is the arguments and results of the procedure. In this case, a transition is completely described by a single entry in a trace, such as (Read (a1), d) .

Now we are ready to consider modules with state. Our idea is to generalize what we did with pairs of states described by single trace entries to sequences of states described by longer traces. Suppose that $T$ and $S$ are any modules that have the same external interface (set of procedures that are exported and hence may be invoked externally). In this discussion, we will often refer to $S$ as the specification module and $T$ as the implementation. Then we say that $T$ implements $S$ if every trace of $T$ is also a trace of $S$. That is, the set of traces generated by $T$ is a subset of the set of traces generated by $S$.

This says that any external behavior of the implementation $T$ must also be an external behavior of the spec $S$. Another way of looking at this is that we shouldn't be able to tell by looking at the implementation that we aren't looking at the spec, so we have to be able to explain every behavior of $T$ as a possible behavior of $S$.

The reverse, however, is not true. We do not insist that the implementation must exhibit every behavior allowed by the spec. In the case of the simple memory the spec is completely deterministic, so the implementations cannot take advantage of this freedom. In general, however, the spec may allow lots of behaviors and the implementation choose just one. The spec for sorting, for instance, allows any sorted sequence as the result of sort; there may be many such sequences if the ordering relation is not total. The implementation will usually be deterministic and return exactly one of them, so it doesn't exhibit all the behavior allowed by the spec.

\section{Safety and liveness}

Just as with procedures, this subset requirement is not strong enough to satisfy our intuitive notion of implementation. In particular, it allows the set of traces generated by $T$ to be empty; in other word, the implementation might do nothing at all, or it might do some things and then stop. As we saw, the analog of this for a simple sequential procedure is non-termination. Usually we want to say that the implementation of a procedure should terminate, and similarly we want to say that the implementation of a module should keep doing things. More generally, when we 
have concurrency we usually want the implementation to be fair, that is, to eventually service all its clients, and more generally to eventually make any transition that continues to be enabled.

It turns out that any external behavior (that is, any set of traces) can be described as the intersection of two sets of traces, one defined by a safety property and the other defined by a liveness property. ${ }^{1}$ A safety property says that in the trace nothing bad ever happens, or more precisely, that no bad transition occurs in the trace. It is analogous to partial correctness for a stateless procedure; a state machine that never makes a bad transition can define any safety property. If a trace doesn't satisfy a safety property, you can always find this out by looking at a finite prefix of the trace, in particular, at a prefix that includes the first bad transition.

A liveness property says that in the trace something good eventually happens. It is analogous to termination for a stateless procedure. You can never tell that a trace doesn't have a liveness property by looking at a finite prefix, since the good thing might happen later. A liveness property cannot be defined by a state machine. It is usual to express liveness properties in terms of fairness, that is, in terms of a requirement that if some transition stays enabled continuously it eventually occurs (weak fairness), or that if some transition stays enabled intermittently it eventually occurs (strong fairness).

With a few exceptions, we don't deal with liveness in this course. There are two reasons for this. First, it is usually not what you want. Instead, you want a result within some time bound, which is a safety property, or you want a result with some probability, which is altogether outside the framework we have set up. Second, liveness proofs are usually hard.

\section{Abstraction functions and simulation}

The definition of 'implements' as inclusion of external behavior is a sound formalization of our intuition. It is difficult to work with directly, however, since it requires reasoning about infinite sets of infinite sequences of actions. We would like to have a way of proving that $T$ implements $S$ that allows us to deal with one of $T$ 's actions at a time. Our method is based on abstraction functions.

An abstraction function maps each state of the implementation $T$ to a state of the specification $S$ For example, each state of the WBCache module gets mapped to a state of the Memory module. The abstraction function explains how to interpret each state of the implementation as a state of the specification. For example, Figure 3 depicts part of the abstraction function from wBCache to Memory. Here is its definition in Spec, copied from handout 5.

FUNC $A F() \rightarrow M=\operatorname{RET}(\backslash a \mid c ! a=c(a)[*] m(a))$

1 B. Alpern and F. Schneider. Recognizing safety and liveness. Distributed Computing 2, 3 (1987), pp 117-126.

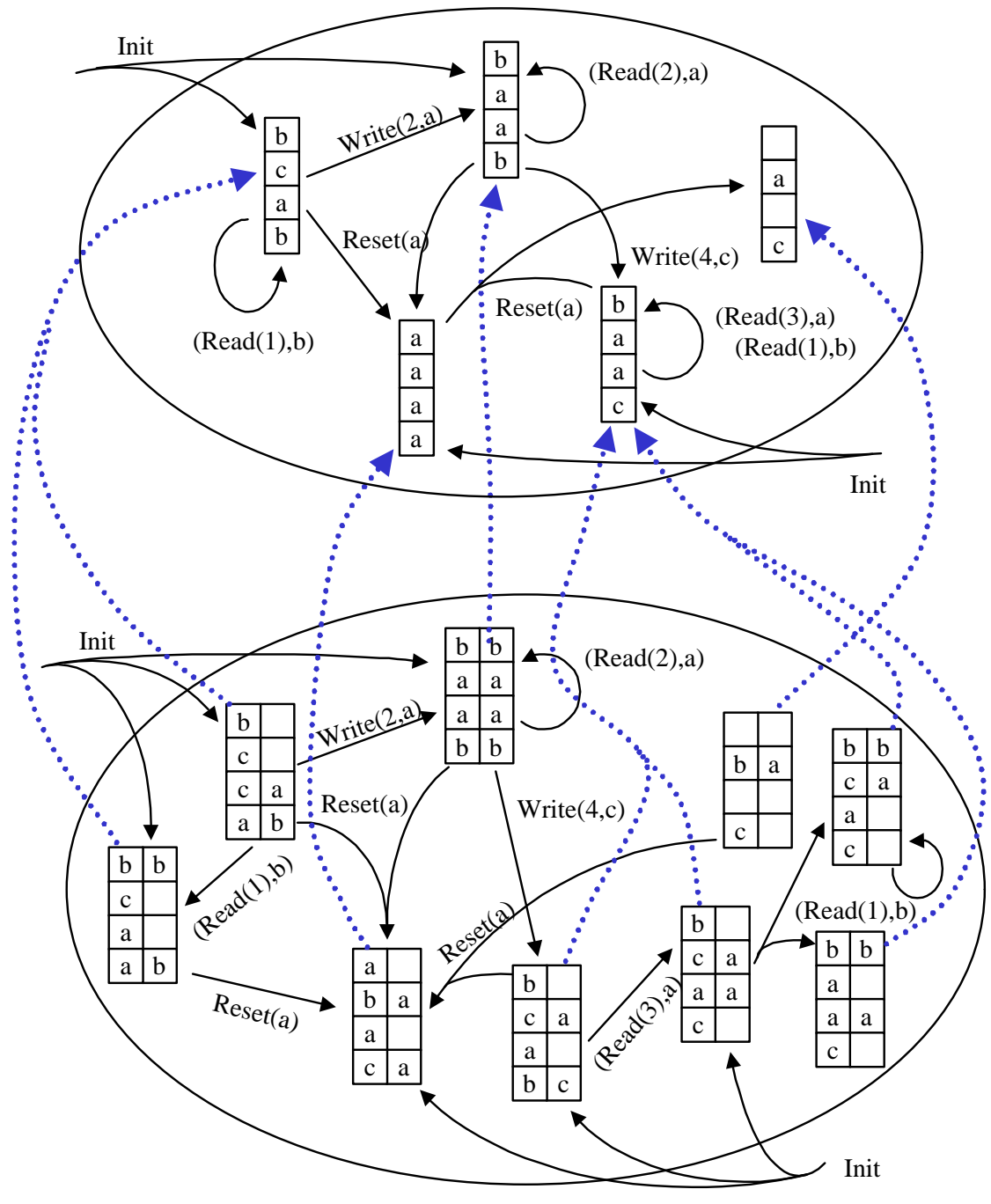

Figure 3: Abstraction function for wBCache 
You might think that an abstraction function should map the other way, from states of the specification to states of the implementation, explaining how to represent each state of the specification. This doesn't work, however, because there is usually more than one way of representing each state of the specification. For example, for the wBCache implementation of Memory, if an address is in the cache, then the value stored for that address in memory does not matter. There are also choices about which addresses appear in the cache. Thus, many states of the implementation can represent the same state of the specification. In other words, the abstraction function is many-to-one.

An abstraction function $F$ is required to satisfy the following two conditions.

1. If $t$ is any initial state of $T$, then $F(t)$ is an initial state of $S$.

2. If $t$ is a reachable state of $T$ and $\left(t, \pi, t^{\prime}\right)$ is a step of $T$, then there is a step of $S$ from $F(t)$ to $F\left(t^{\prime}\right)$, having the same trace.

Condition 2 says that $T$ simulates $S$; every step of $T$ faithfully copies a step of $S$. It is stated in a particularly simple way, forcing the given step of $T$ to simulate a single step of $S$. That is enough for the special case we are considering right now. Later, when we consider concurrent invocations for modules, we will generalize condition 2 to allow any number of steps of $S$ rather than just a single step.

The diagram in Figure 4 represents condition 2. The dashed arrows represent the abstraction function $F$, and the solid arrows represent the transitions; if the lower (double) solid arrow exists in the implementation, the upper (single) solid arrow must exist in the specification. The diagram is sometimes called a "commutative diagram" because if you start at the lower left and follow arrows, you will end up in the same state regardless of which way you go.

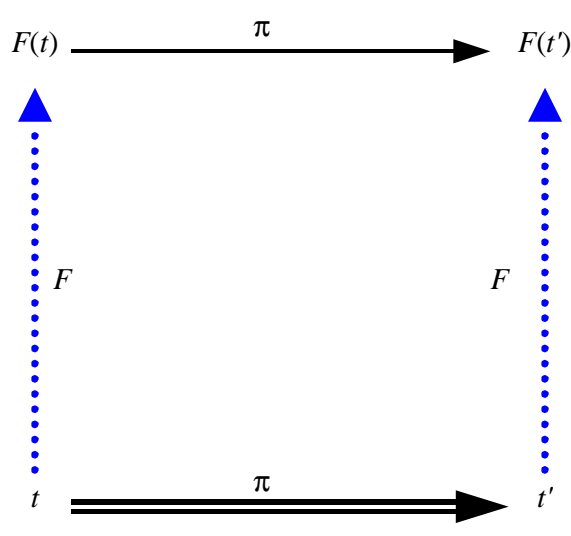

Figure 4: Commutative diagram for correctness
An abstraction function is important because it can be used to show that one module implements another:

Theorem 1: If there is an abstraction function from $T$ to $S$, then $T$ implements $S$, i.e., every trace of $T$ is a trace of $S$.

Note that this theorem applies to both finite and infinite traces.

Proof: (Sketch) Let $\beta$ be any trace of $T$, and let $\alpha$ be any execution of $T$ that generates trace $\beta$. Use Conditions 1 and 2 above to construct an execution $\alpha^{\prime}$ of $S$ with the same trace. That is, if $t$ is the initial state of $\alpha$, then let $F(t)$ be the initial state of $\alpha^{\prime}$. For each step of $\alpha$ in turn, use Condition 2 to add a corresponding step to $\alpha^{\prime}$.

More formally, this proof is an induction on the length of the execution. Condition 1 gives the basis: any initial state of $T$ maps to an initial state of $S$. Condition 2 gives the inductive step: if we have an execution of $T$ of length $n$ that simulates an execution of $S$, any next step by $T$ simulates a next step by $S$, so any execution of $T$ of length $n+1$ simulates an execution of $S$

We would like to have an inverse of Theorem 1: if every trace of $T$ is a trace of $S$, then there is an abstraction function that shows it. This is not true for the simple abstraction functions and simulations we have defined here. Later on, in handout 8 , we will generalize them to a simulation method that is strong enough to prove that $T$ implements $S$ whenever that is true.

\section{Invariants}

An invariant of a module is any property that is true of all reachable states of the module, i.e., all states that can be reached in executions of the module (starting from initial states). Invariants are important because condition 2 for an abstraction function requires us to show that the implementation simulates the spec from every reachable state, and the invariants characterize the reachable states. It usually isn't true that the implementation simulates the spec from every state.

Here are examples of invariants for the HashMemory and MajorityRegister modules, written in Spec and copied from handout 5.

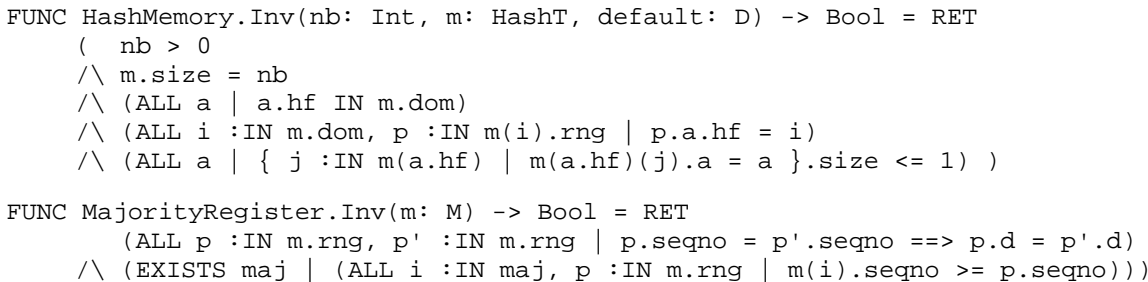

For example, for the HashMemory module, the invariant says (among other things) that a pair containing address a appears only in the appropriate bucket a.hf, and that at most one pair for an address appears in the bucket for that address.

The usual way to prove that a property $P$ is an invariant is by induction on the length of finite executions leading to the states in question. That is, we must show the following: 
(Basis, length =0) $P$ is true in every initial state.

(Inductive step) If $\left(t, \pi, t^{\prime}\right)$ is a transition and $P$ is true in $t$, then $P$ is also true in $t^{\prime}$.

Not all invariants are proved directly by induction, however. It is often better to prove invariants in groups, starting with the simplest invariants. Then the proofs of the invariants in the later groups can assume the invariants in the earlier groups.

Example: We sketch a proof that the property MajorityRegister.Inv is in fact an invariant. Basis: In any initial state, a single (arbitrarily chosen) default value $d$ appears in all the copies, along with the seqno 0 . This immediately implies both parts of the invariant.

Inductive step: Suppose that $\left(t, \pi, t^{\prime}\right)$ is a transition and Inv is true in $t$. We consider cases based on $\pi$. If $\pi$ is an invocation or response, or the body of a Read procedure, then the step does not affect the truth of Inv. So it remains to consider the case where $\pi$ is a write, say write (d) .

In this case, the second part of the invariant for $t$ (i.e., the fact that the highest seqno appears in more than half the copies), together with the fact that the write reads a majority of the copies, imply that the Write obtains the highest segno, say $i$. Then the new segno that the write

chooses must be the new highest seqno. Since the write writes $i+1$ to a majority of the copies, it ensures the second part of the invariant. Also, since it associates the same d with the segno $i+1$ everywhere it writes, it preserves the first part of the invariant.

\section{Proofs using abstraction functions}

Example: We sketch a proof that the function WBCache. AF given above is an abstraction function from WBCache to Memory. In this proof, we get by without any invariants.

For Condition 1, suppose that $t$ is any initial state of WBCache. Then $\mathrm{AF}(t)$ is some (memory) state of Memory. But all memory states are allowable in initial states of Memory. Thus, $A F(t)$ is an initial state of Memory, as needed. For Condition 2, suppose that $t$ and $\mathrm{AF}(t)$ are states of wBCache and Memory, respectively, and suppose that $\left(t, \pi, t^{\prime}\right)$ is a step of wBCache. We consider cases, based on $\pi$.

For example, suppose $\pi$ is Read (a). Then the step of wBCache may change the cache and memory by writing a value back to memory. However, these changes don't change the corresponding abstract memory. Therefore, the memory correspondence given by AF holds after the step. It remains to show that both Reads give the same result. This follows because:

The Read (a) in wBCache returns the value $t . c(a)$ if it is defined, otherwise $t . m(a)$.

The Read (a) in Memory returns the value of $\operatorname{AF}(t) \cdot m(a)$.

The value of $\operatorname{AF}(t) \cdot \mathrm{m}(\mathrm{a})$ is equal to $t . \mathrm{c}(\mathrm{a})$ if it is defined, otherwise $t \cdot \mathrm{m}(\mathrm{a})$. This is by the definition of $\mathrm{AF}$.

For another example, suppose $\pi$ is Write $(a, d)$. Then the step of wBCache writes value $d$ to location a in the cache. It may also write some other value back to memory. Since writing a value back does not change the corresponding abstract state, the only change to the abstract state is that the value in location a is changed to $d$. On the other hand, the effect of Write $(a, d)$ in Memory is to change the value in location a to $\mathrm{d}$. It follows that the memory correspondence, given by $\mathrm{AF}$, holds after the step.

We leave the other cases, for the other types of operations, to the reader. It follows that $A F$ is an abstraction function from WBCache to Memory. Then Theorem 1 implies that WBCache implements Memory, in the sense of trace set inclusion.

Example: Here is a similar analysis for MajorityRegister, using MajorityRegister. $\mathrm{AF}$ as the abstraction function.

FUNC $A F() \rightarrow D=R E T$ m.rng.fmax $((\backslash p 1, p 2 \mid p 1$.seqno $<=p 2$.seqno $)) \cdot d$

This time we depend on the invariant MajorityRegister. Inv. Suppose $\pi$ is Read (a). No state changes occur in either module, so the only thing to show is that the return values are the same in both modules. In MajorityRegister, the Read collects a majority of values and returns a value associated with the highest segno from among that majority. By the invariant that says that the highest seqno appears in a majority of the copies, it must be that the Read in fact obtains the highest segno that is present in the system. That is, the Read in MajorityRegister returns a value associated with the highest seqno that appears in state $t$.

On the other hand, the Read in Register just returns the value of the single variable $\mathrm{x}$ in state $s$. Since $\operatorname{AF}(t)=s$, it must be that $s . x$ is a value associated with the highest seqno in $t$. But the uniqueness invariant says that there is only one such value, so this is the same as the value returned by the Read in MajorityRegister.

Now suppose $\pi$ is Write (d). Then the key thing to show is that $A F\left(t^{\prime}\right)=s^{\prime}$. The majority invarian implies that the Write in MajorityRegister sees the highest segno $i$ and thus $i+1$ is the new highest seqno. It writes $(i+1, d)$ to a majority of the copies. On the other hand, the write in Register just sets $\mathrm{x}$ to $\mathrm{d}$. But clearly d is a value associated with the largest segno after the step, $\operatorname{so} \operatorname{AF}\left(t^{\prime}\right)=s^{\prime}$ as required.

It follows that $A F$ is an abstraction function from MajorityRegister to Register. Then Theorem 1 implies that MajorityRegister implements Register. 



\section{Disks and File Systems}

\section{Motivation}

The two lectures on disks and file systems are intended to show you a number of things:

Some semi-realistic examples of specs.

Many important implementation techniques for file systems.

Some of the tradeoffs between a simple spec and an efficient implementation.

Examples of abstraction functions and invariants.

Encoding: a general technique for representing arbitrary types as byte sequences.

How to model crashes.

Transactions: a general technique for making big actions atomic.

There are a lot of ideas here. After you have read this handout and listened to the lectures, it's a good idea to go back and reread the handout with this list of themes in mind.

\section{Outline of topics}

We give the specifications of disks and files in the Disk and File modules, and we discuss a variety of implementation issues:

Crashes

Disks

Files

Caching and buffering of disks and files

Representing files by trees and extents

Allocation

Encoding and decoding

Directories

Transactions

Redundancy

\section{Crashes}

The specs and implementations here are without concurrency. However, they do allow for crashes. A crash can happen between any two atomic commands. Thus the possibility of crashes introduces a limited kind of concurrency.
When a crash happens, the volatile global state is reset, but the stable state is normally unaffected. We express precisely what happens to the global state as well as how the module recovers by including a Crash procedure in the module. When a crash happens:

1. The Crash procedure is invoked. It need not be atomic.

2. If the Crash procedure does a CRASH command, the execution of the current invocations (if any) stop, and their local state is discarded; the same thing happens to any invocations outside the module from within it. After CRASH, no procedure in the module can be invoked from outside until crash returns.

3. The Crash procedure may do other actions, and eventually it returns.

4. Normal operation of the module resumes; that is, external invocations are now possible.

You can tell which parts of the state are volatile by looking at what Crash does; it will reset the volatile variables.

Because crashes are possible between any two atomic commands, atomicity is important for any operation that involves a change to stable state.

The meaning of a Spec program with this limited kind of concurrency is that each atomic command corresponds to a transition. A hidden piece of state called the program counter keeps track of what transitions are enabled next: they are the atomic commands right after the program counter. There may be several if the command after the program counter has [] as its operator. In addition, a crash transition is always possible; it resets the program counter to a null value from which no transition is possible until some external routine is invoked and then invokes the Crash routine.

If there are non-atomic procedures in the spec with many atomic commands, it can be rather difficult to see the consequences of a crash. It is therefore clearer to write a spec with as much atomicity as possible, making it explicit exactly what unusual transitions are possible when there's a crash. We don't always follow this style, but we give some examples of it, notably at the end of the section on disks.

\section{Disks}

Essential properties of a disk:

Storage is stable across crashes (we discuss error models for disks in the Disk spec).

It's organized in blocks, and the only atomic update is to write one block.

Random access is about 100k times slower than random access to RAM (10 ms vs. $100 \mathrm{~ns}$ )

Sequential access is 10-50 times slower than sequential access to RAM (20 MB/s vs. 200$1000 \mathrm{MB} / \mathrm{s}$ )

Costs 50 times less than RAM (\$.01 MB vs. \$1/MB from the back of a PC magazine) in January 2000.

MTBF 1 million hours $=100$ years 


\section{Performance numbers}

Blocks of $.5 \mathrm{k}-4 \mathrm{k}$ bytes

$20 \mathrm{MB} / \mathrm{sec}$ sequential, sustained (more with parallel disks)

$3 \mathrm{~ms}$ average rotational delay $(10000 \mathrm{rpm}=6 \mathrm{~ms}$ rotation time)

$7 \mathrm{~ms}$ average seek time; $3 \mathrm{~ms}$ minimum

It takes $10 \mathrm{~ms}$ to get anything at all from a random place on the disk. In another $10 \mathrm{~ms}$ you can transfer $200 \mathrm{~KB}$. Hence the cost to get $200 \mathrm{~KB}$ is only twice the cost to get 1 byte. By reading from several disks in parallel (called striping or RAID) you can easily increase the transfer rate by a factor of 5-10.

\section{Performance techniques:}

Avoid disk operations: use caching

Do sequential operations: allocate contiguously, prefetch, write to log

Write in background (write-behind)

\section{A spec for disks}

The following module describes a disk Dsk as a function from a DA to a disk block DB, which is just a sequence of DBSize bytes. The Dsk function can also yield nil, which represents a

permanent read error. The module is a class, so you can instantiate as many Disks as needed.

The state is one Dsk for each Disk. There is a New method for making a new disk; think of this as ordering a new disk drive and plugging it in. An extent $\mathrm{E}$ represents a set of consecutive disk addresses. The main routines are the read and write methods of Disk: read, which reads an extent, and write, which writes $n$ disk blocks worth of data sequentially to the extent $E\{d a, n\}$. The write is not atomic, but can be interrupted by a failure after each single block is written.

Usually a spec like this is written with a concurrent thread that introduces permanent errors in the recorded data. Since we haven't discussed concurrency yet, in this spec we introduce the errors in reads, using the AddErrors procedure. An error sets a block to nil, after which any read that includes that block raises the exception error. Strictly speaking this is illegal, since read is a function and therefore can't call the procedure AddErrors. When we learn about concurrency we can move AddErrors to a separate thread; in the meantime we take the liberty, since it would be a real nuisance for read to be a procedure rather than a function.

Since neither Spec nor our underlying model deals with probabilities, we don't have any way to say how likely an error is. We duck this problem by making AddErrors completely nondeterministic; it can do anything from introducing no errors (which we must hope is the usual case) to clobbering the entire disk. Characterizing errors would be quite tricky, since disks usually have at least two classes of error: failures of single blocks and failures of an entire disk. However, any user of this module must assume something about the probability and distribution of errors.

Transient errors are less interesting because they can be masked by retries. We don't model them, and we also don't model errors reported by writes. Finally, a realistic error model would include the possibility that a block that reports a read error might later be readable after all.
CLASS Disk EXPORT Byte, Data, DA, E, DBSize, read, write, size, check, Crash=

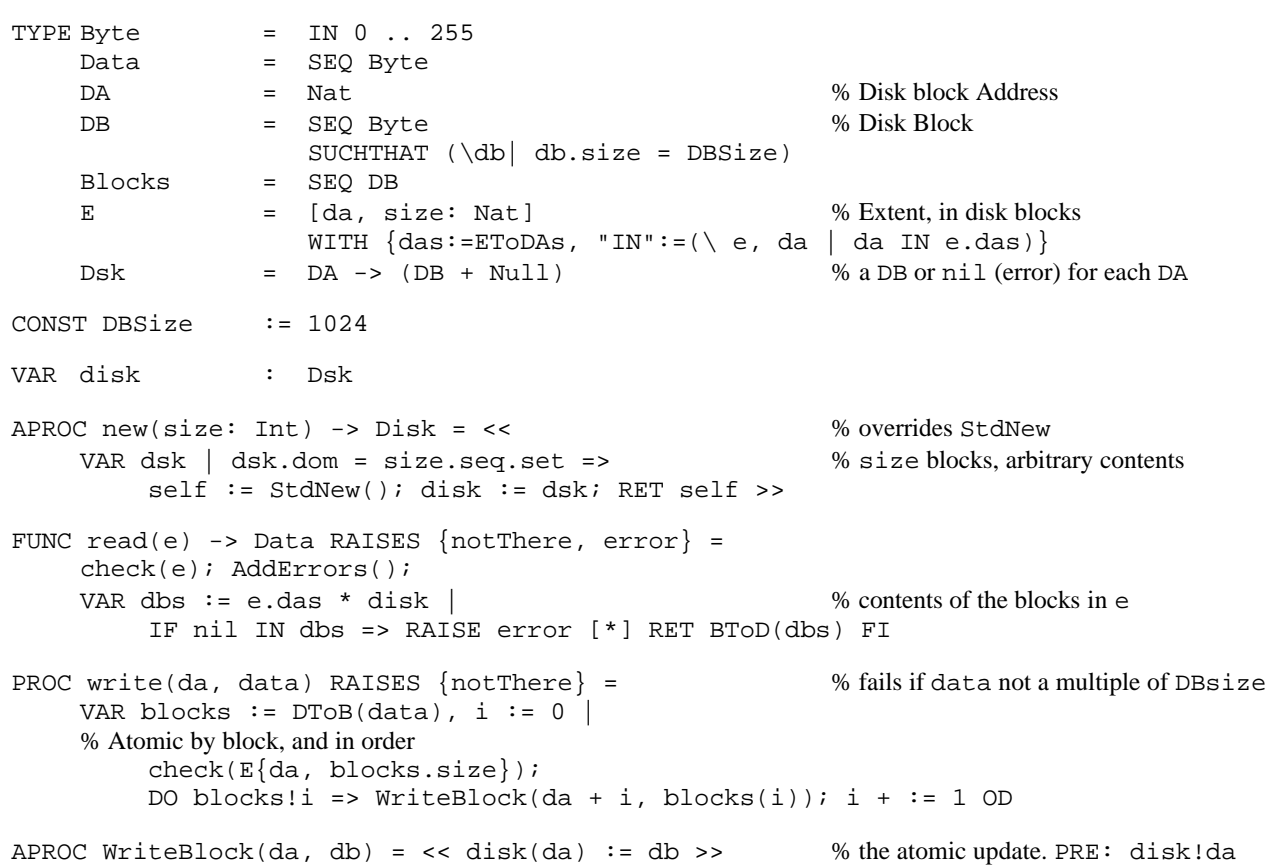

\section{\% Internal routines}

$\%$ Functions to convert between Data and Blocks.

FUNC BToD (blocks) $\rightarrow$ Data = RET + . blocks

FUNC DTOB (data) $\rightarrow$ Blocks = VAR blocks $\mid$ BTOD (blocks) = data $\Rightarrow$ RET blocks $\%$ Undefined if data.size is not a multiple of DBsize

APROC AddErrors () =

$\%$ clobber some blocks

< DO RET [] VAR da :IN disk.dom | disk(da) := nil OD $>>$ 
This module doesn't worry about the possibility that a disk may fail in such a way that the client can't tell whether a write is still in progress; this is a significant problem in fault tolerant systems that want to allow a backup processor to start running a disk as soon as possible after the primary fails.

Many disks do not guarantee the order in which blocks are written (why?) and thus do not implement this spec, but instead one with a weaker write:

PROC writeUnordered(da, data) RAISES \{notThere $=$

VAR blocks $:=$ DTOB (data) |

$\%$ Atomic by block, in arbitrary order; assumes no concurrent writing.

check (E\{da, blocks.size $\})$;

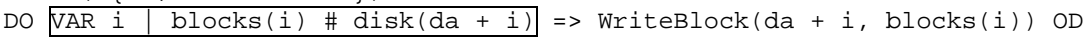

In both specs write establishes blocks $=\mathrm{E}\{\mathrm{da}$, blocks.size $\}$.das $*$ disk, which is the same as data $=\operatorname{read}(\mathrm{E}\{\mathrm{da}, \mathrm{blocks.size}\})$, and both change each disk block atomically. writeUnordered says nothing about the order of changes to the disk, so after a crash any subset of the blocks being written might be changed; write guarantees that the blocks changed are a prefix of all the blocks being written. (writeUnordered would have other differences from write if concurrent access to the disk were possible, but we have ruled that out for the moment.)

\section{Clarifying crashes}

In this spec, what happens when there's a crash is expressed by the fact that write is not atomic and changes the disk one block at a time in the atomic WriteBlock. We can make this more explicit by making the occurrence of a crash visible inside the spec in the value of the crashed variable. To do this, we modify crash so that it temporarily makes crashed true, to give write a chance to see it. Then write can be atomic; it writes all the blocks unless crashed is true, in which case it writes some prefix; this will happen only if write is invoked between the crashed := true and the CRASH commands of Crash. To describe the changes to the disk neatly, we introduce an internal function NewDisk that maps a dsk value into another one in which disk blocks at da are replaced by corresponding blocks defined in bs.

Again, this wouldn't be right if there were concurrent accesses to Disk, since we have made all the changes atomically, but it gives the possible behavior if the only concurrency is in crashes.

VAR crashed : Bool := false

..

APROC write (da, data) RAISES $\{$ notThere $\}=k<$

VAR blocks := DTOB (data)

check (E\{da, blocks.size $\}) ;$
$\begin{aligned} & \text { IF Crashed }=> \\ & \text { VAR } i \mid i<\text { blocks.size }=>\text { blocks }:=\text { blocks.sub( }(0, i) \\ & {\left[{ }^{*}\right] \text { SKIP FI; }} \\ & \text { disk := NewDisk (disk, da, blocks) }\end{aligned}$

$$
\begin{aligned}
& {\left[{ }^{*}\right]} \\
& \text { disk } \quad:=\text { NewIP FI; }
\end{aligned}
$$

FUNC NewDisk (dsk, da, bs: (Int $\rightarrow$ DB)) $\rightarrow$ Dsk $=$

$\operatorname{RET} \mathrm{dsk}+\left(\backslash \mathrm{da} a^{\prime} \mid \mathrm{da} \mathbf{a}^{\prime}-\mathrm{da}\right) *$ bs
PROC Crash ()$=$ Crashed $:=$ true; CRASH; crashed $:=$ false

For unordered writes we need only a slight change, to write an arbitrary subset of the blocks if there's a crash, rather than a prefix:

IF crashed $=>$

$\%$ if crashed, write some subset VAR \begin{tabular}{|l|l|} 
w: SET I & w $<=$ blocks.dom
\end{tabular}$=>$ blocks $:=$ blocks. restrict $(\mathrm{w})$

\section{Files}

This section gives a variety of specifications for files. Implementations follow in later sections.

We treat a file as just a sequence of bytes. Files have names, and for now we confine ourselves to a single directory that maps names to files. We call the name a 'path name' PN with an eye toward later introducing multiple directories, but for now we just treat the path name as a string without any structure. We package the operations on files as methods of PN. The main methods are read and write; we define the latter initially as WriteAtomic, and later introduce less atomic variations write and writeUnordered. There are also boring operations that deal with the size and with file names.

MODULE File EXPORT PN, Byte, Data, X, F, Crash =

TYPE PN

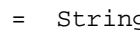
$\%$ Path Name

WITH read:=Read, write:=WriteAtomic, size:=GetSize, setSize:=SetSize, create:=Create, remove:=Remove, rename: =Rename

Byte $\quad$ IN $\quad$ Int 255

Data $\quad$ SEQ Byte

$\mathrm{X} \quad \mathrm{Nat} \quad \%$ byte-in-file indeX

$\mathrm{F} \quad=$ Data $\%$ File

Dir $\quad=\mathrm{PN} \rightarrow \mathrm{F} \quad \%$ Directory

VAR dir := Dir \{\}$\quad \%$ undefined everywhere

Note that the only state of the spec is dir, since files are only reachable through dir.

There are tiresome complications in write caused by the fact that the arguments may extend beyond the end of the file. These can be handled by imposing preconditions (that is, writing the spec to do HAVOC when the precondition isn't satisfied), by raising exceptions, or by defining some sensible behavior. This spec takes the third approach; NewFile computes the desired contents of the file after the write. So that it will work for unordered writes as well, it handles sparse data by choosing an arbitrary data' that agrees with data where data is defined. Compare it with Disk. NewDisk.

FUNC Read(pn, $x, i) \rightarrow$ Data $=\operatorname{RET} \operatorname{dir}(p n) \cdot \operatorname{seg}(x, i)$

$\%$ Returns as much data as available, up to $i$ bytes, starting at $x$.

APROC WriteAtomic $(p n, x, \operatorname{data})=\langle<\operatorname{dir}(p n):=$ NewFile(dir(pn), $x$, data) $>>$ 
FUNC NewFile(f0, $x$, data: Int $\rightarrow$ Byte) $->F=$

$\% \mathrm{f}$ is the desired final file. Fill in space between $\mathrm{f} 0$ and $\mathrm{x}$ with zeros, and undefined data elements arbitrarily. VAR $z:=$ data.dom.max, $z 0:=$ fo.size, f, data'
data'.size = z $八$ data'.restrict (data.dom) = data

f.size $=\{z 0, x+z\} \cdot \max$

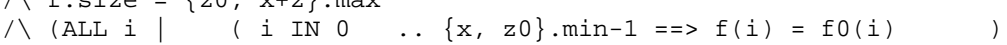

八 $\left(\begin{array}{llllll}i & \text { IN } & z 0 & \ldots & x-1 & =\end{array} \mathrm{f}(i)=0\right.$

$八\left(\begin{array}{ccccc}i \text { IN } x & \ldots x+z-1 \quad==f(i)=\operatorname{data}^{\prime}(i-x)\end{array}\right)$

$\Rightarrow$ RET $\mathrm{f}$

FUNC GetSize(pn) $\rightarrow X=$ RET $\operatorname{dir}(p n)$.size

APROC SetSize $(p n, x)=<\operatorname{VAR} z:=$ pn.size

IF $x<=z=><\operatorname{dir}(p n):=\operatorname{pn} . \operatorname{read}(0, z)>>$

[*] pn.write $(z$, F.fill $(0, x-z+1))$

FI $>$

\section{$\%$ truncate}

$\%$ handles crashes like write

APROC Create $(\mathrm{pn})=\langle<\operatorname{dir}(\mathrm{pn}):=\mathrm{F}\{\}>>$

APROC Remove $(\mathrm{pn})=\langle<\operatorname{dir}:=\operatorname{dir}\{\mathrm{pn} \rightarrow\}\rangle>$

APROC Rename (pn1, pn2) $=\langle<\operatorname{dir}(p n 2):=\operatorname{dir}(p n 1) ;$ Remove (pn1) $\rangle>$

$\operatorname{PROC} \operatorname{Crash}()=\operatorname{SKIP}$

$\%$ no volatile state or non-atomic changes

\section{END File}

WriteAtomic changes the entire file contents at once, so that a crash can never leave the file in an intermediate state. This would be quite expensive in most implementations. For instance,

consider what is involved in making a write of 20 megabytes to an existing file atomic; certainly you can't overwrite the existing disk blocks one by one. For this reason, real file systems don't implement writeAtomic. Instead, they change the file contents a little at a time, reflecting the fact that the underlying disk writes blocks one at a time. Later we will see how an atomic write could be implemented in spite of the fact that it takes several atomic disk writes. In the meantime, here is a more realistic spec for Write that writes the new bytes in order. It is just like Disk.write except for the added complication of extending the file when necessary, which is taken care of in NewFile.

APROC Write $(\mathrm{pn}, \mathrm{x}$, data $)=<<$

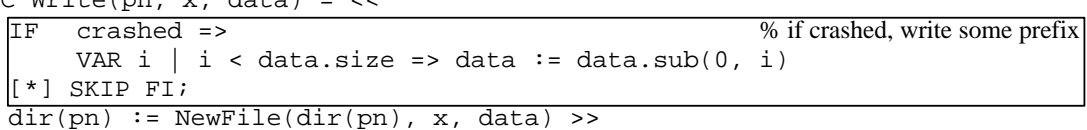

PROC Crash ()$=$ crashed $:=$ true; CRASH; crashed $:=$ false

This spec reflects the fact that only a single disk block can be written atomically, so there is no guarantee that all of the data makes it to the file before a crash. At the file level it isn't appropriate to deal in disk blocks, so the spec promises only bytewise atomicity. An actually implementation would probably make changes one page at a time, so it would not exhibit all the behavior allowed by the spec. There's nothing wrong with this, as long as the spec is restrictive enough to satisfy its clients.

Write does promise, however, that $f(i)$ is changed no later than $f(i+1)$. Some file systems make no ordering guarantee; for them the following WriteUnordered is appropriate; it is just like Disk.writeUnordered.

APROC WriteUnordered (pn, $\mathrm{x}$, data) $=<<$

IF crashed $=>$ restrict $(w)$ * VAR W:

$\operatorname{dir}(\mathrm{pn}):=$ NewFile $(\operatorname{dir}(\mathrm{pn}), \mathrm{x}, \mathrm{data})>>$

Notice that although writing a file is not atomic, File's directory operations are atomic. This corresponds to the semantics that file systems usually attempt to provide: if there is a failure during a Create, Remove, or Rename, the operation is either completed or not done at all, but if there is a failure during a Write, any amount of the data may be written. The other reason for making this choice in the spec is simple: with the abstractions available there's no way to express any sensible intermediate state of a directory operation other than Rename (of course a sloppy implementation might leave the directory scrambled, but that has to count as a bug; think what it would look like in the spec).

The spec we gave for SetSize made it as atomic as write. The following spec for SetSize is unconditionally atomic; this might be appropriate because an atomic setsize is easier to implement than a general atomic Write:

$\operatorname{APROC} \operatorname{SetSize}(p n, x)=\langle<\operatorname{dir}(p n):=(\operatorname{dir}(p n)+F \cdot f i l l(0, x)) \cdot \operatorname{seg}(0, x)>>$

Here is another version of NewFile, written in a more operational style just for comparison. It is a bit shorter, but less explicit about the relation between the initial and final states.

FUNC NewFile(f0, $x$, data: Int $\rightarrow$ Byte) $\rightarrow F=$ VAR z0 $:=$ f0.size, data' data'.size $=$ data.dom.max $=>$

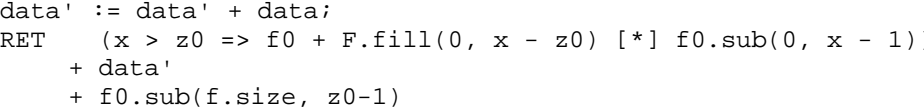

Our File spec is missing some things that are important in real file systems:

Access control: permissions or access control lists on files, ways of defaulting these when a file is created and of changing them, an identity for the requester that can be checked against the permissions, and a way to establish group identities.

Multiple directories. We will discuss this when we talk about naming.

Quotas, and what to do when the disk fills up.

Multiple volumes or file systems.

Backup. We will discuss this near the end of this handout when we describe the copying file system. 


\section{Cached and buffered disks}

The simplest way to decouple the file system client from the slow disk is to provide a cached and write buffered implementation of the Disk abstraction; then the file system implementation need not change. The basic ideas are very similar to the ideas for cached memory, although for the disk we preserve the order of writes. We didn't do this for the memory because we didn't worry about failures.

Failures add complications; in particular, the spec must change, since buffering writes means that some writes may be lost if there is a crash. Furthermore, the client needs a way to ensure that its writes are actually stable. We therefore need a new spec BDisk. To get it, we add to Disk a

variable $\circ$ ldDisks that remembers the previous states that the disk might revert to after a crash (note that this is not necessarily all the previous states) and code to use oldDisks appropriately. BDisk . write no longer needs to test crashed, since it's now possible to lose writes even if the crash happens after the write.

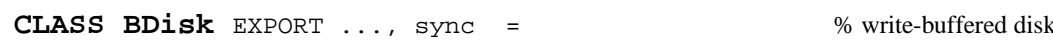

TYPE ...
VAR disk
$\%$ as in Disk

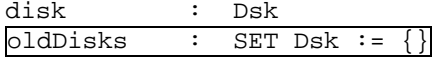

APROC write (da, data) RAISES $\{$ notThere $\}=<<$

$<<$ VAR blocks := DTOB (data)

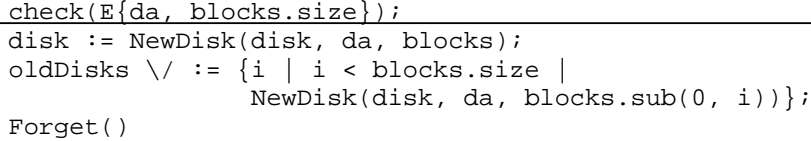

$\%$ result is dsk overwritten with bs at da

TET dsk + ( $($ da' $\mid$ da' - da $) *$ bs

PROC sync() = OldDisks $:=\{\}$
PROC Forget ()$=$ VAR ds: SET Dsk | OldDisks $-:=d s$
\% Discards an arbitrary subset of the remembered disk states.

$\%$ Discards an arbitrary subset of the remembered disk states.

PROC $\operatorname{Crash}()=$ CRASH; $<<$ VAR d : IN OldDisks $\mid$ disk $:=d$; sync() $[*]$ SKIP $>>$

END BDisk

Forget is there so that we can write an abstraction function for an implementation that doesn' defer all its disk writes until they are forced by Sync. A write that actually changes the disk needs to change oldDisks, because oldDisks contains the old state of the disk block being overwritten, and there is nothing in the state of the implementation after the write from which to compute that old state. Later we will study a better way to handle this problem: history variables or multi-valued mappings. They complicate the implementation rather than the spec, which is preferable. Furthermore, they do not affect the performance of the implementation at all.
A weaker spec would revert to a state in which any subset of the writes has been done. For this, change the assignment to oldDisks in write, along the lines we have seen before.

$$
\text { oldDisks } \backslash /:=\frac{\{\text { W: SET I } \mid \text { w }<=\text { blocks.dom } \mid}{\text { NewDisk(disk, da, blocks. restrict (w) })\} ; ; ~}
$$

The module BufferedDisk below is an implementation of BDisk. It copies newly written data into the cache and does the writes later, preserving the original order so that the state of the disk after a crash will always be the state at some time in the past. In the absence of crashes this implements Disk and is completely deterministic. We keep track of the order of writes with a queue variable, instead of keeping a dirty bit for each cache entry as we did for cached memory. If we didn't do the writes in order, there would be many more possible states after a crash, and it would be much more difficult for a client to use this module. Many real disks have this unpleasant property, and many real systems deal with it by ignoring it.

A striking feature of this implementation is that it uses the same abstraction that it implements, namely BDisk. The implementation of BDisk that it uses we call UDisk (U for 'underlying'). We think of it as a 'physical' disk, and of course it is quite different from BufferedDisk: it contains SCSI controllers, magnetic heads, etc. A module that implements the same interface that it use is sometimes called a filter or a stackable module. A Unix filter is a familiar example that uses and implements the byte stream interface. We will see many other examples of this in the course.

Invocations of UDisk are in bold type, so you can easily see how the module depends on the lower-level implementation of BDisk.

\section{CLASS BufferedDisk}

$\%$ implements BDisk

EXPORT Byte, Data, DA, E, DBSize, read, write, size, check, sync, Crash =

TYPE \% Data, DA, DB, Blocks, E as in Disk

$$
\begin{aligned}
& \text { I }=\text { Int } \\
& \text { Queue } \quad=\quad S E Q D A \\
& \text { cacheSize }:=1000 \\
& \text { queueSize } \quad:=50 \\
& \text { VAR udisk : Disk } \\
& \text { cache : DA } \rightarrow D B:= \\
& \text { quene } \quad:=\text { Queue }\{\}
\end{aligned}
$$$$
\text { CONST }
$$

ABSTRACTION FUNCTION bdisk.disk = udisk.disk + cache $\therefore$ ABSTRACTION FUNCTION bdisk.oldDisks =

$$
\{\mathrm{q} \text { : Queue } \mid \mathrm{q}<=\text { queue | udisk.disk + cache.restrict(q.set) \} }
$$

$\because$ INVARIANT queue.set $<=$ cache.dom

$\because$ INVARIANT queue.size $=$ queue.set.size

$\%$ if queued then cached

- INVARIANT cache dom size $<=$ cachesize

$\therefore$ INVARIANT queue.size $<=$ queueSize

$\%$ no duplicates in queu

$\%$ cache not too big

APROC new(size: Int) $\rightarrow$ BDisk $=<<$

$\%$ queue not too big

self $:=$ StdNew $($ ) i udisk $:=$ udisk.new (size); RET self $>>$ 
PROC $\operatorname{read}(e)->$ Data RAISES \{notThere\} $=$

$\%$ We could make provision for read-ahead, but do not.

check $(e)$;

VAR data $:=$ Data \{\} , da $:=$ e.da, upTo $:=$ e.da + e.size |

DO da < upTo $=>$

IF cache!da $\Rightarrow$ data $+:=$ cache (da); da $+:=1$

$\left[{ }^{*}\right] \div$ read as many blocks from disk as possible

VAR $i:=$ RunNotInCache (da, upTo), buffer := udisk.read $(\mathrm{E}\{\mathrm{da}, i\})$

$\mathrm{k}:=$ MakeCacheSpace (i)

$\% \mathrm{k}$ blocks will fit in cache; add them.

DO VAR $j:$ IN k.seq $\mid \sim$ cache! $(\mathrm{da}+j) \Rightarrow$

cache $(d a+j):=$ udisk.DToB (buffer) $(j)$

FI

$\mathrm{OD}$

OD; RET data

PROC write(da, data) RAISES \{notThere\} =

VAR blocks := udisk.DToB (data)

check ( $E\{d a$, blocks.size $\}$ );

DO VAR i : IN queue.dom | queue(i) IN da .. da+size-1 $\Rightarrow$ Flushqueue (i) OD;

$\%$ Do any previously buffered writes to these addresses. Why?

VAR $j:=$ MakeCacheSpace (blocks, size), $i:=0$

IF $j<$ blocks.size $=>$ udisk. write (da, data)

$\%$ Don't cache if the write is bigger than the cache.

[*] DO blocks!i $\Rightarrow$

$\mathrm{OD}$

cache $($ da $+i):=$ blocks $(i)$; queue $+:=\{$ dati $\} ;+:=1$

PROC Sync() = FlushQueue (queue.size -1 )

PROC Crash ()$=$ CRASH; cache $:=\{\}$; queue $:=\{\}$

FUNC RunNotInCache (da, upTo: DA) $\rightarrow I=$

RET $\{i \mid d a+i<=$ upTo $八$ (ALL $j$ : IN $i . s e q \mid \sim$ cache! $(d a+j)\}$. max

PROC MakeCacheSpace (i) $\rightarrow$ Int $=$

$\%$ Make room for $i$ new blocks in the cache; returning min ( $i$, the number of blocks now available).

$\%$ May flush queue entries.

\% POST: cache.dom.size + result <= cacheSize$$
\text { . . }
$$

PROC Flushqueue $(i)=\operatorname{VAR} q:=$ queue. $\operatorname{sub}(0$, i) $\mid$

$\%$ Write queue entries $0 \ldots i$ and remove them from queue.

$\%$ Should try to combine writes into the biggest possible writes

DO $q \#\{\}=>$ udisk. write (q.head, 1); q := q.tail OD

queue := queue.sub $(i+1$, queve.size -1$)$

END BufferedDisk

This code keeps the cache as full as possible with the most recent data, except for gigantic writes. It would be easy to change it to make non-deterministic choices about which blocks to keep in the cache, or to take advice from the client about which blocks to keep. The latter would require changing the interface to accept the advice, of course.
Note that the only state of BDisk that this module can actually revert to after a crash is the one in which none of the queued writes has been done. You might wonder, therefore, why the body of the abstraction function for BDisk.oldDisks has to involve queue. Why can't it just be

\{udisk. disk\}? The reason is that when the internal procedure Flushqueue does a write, it changes the state that a crash reverts to, and there's no provision in the BDisk spec for adding anything to oldDisks except during write. So oldDisks has to include all the states that the disk can reach after a sequence of 'internal' writes, that is, writes done in FlushQueue. And this is just what the abstraction function says.

Building other kinds of disks

There are other interesting and practical ways to implement a disk abstraction on top of a 'base' disk. Some examples that are used in practice:

Mirroring: use two base disks of the same size to implement a single disk of that size, but with much greater availability and twice the read bandwidth, by doing each write to both base disks.

Striping: use $n$ base disks to implement a single disk $n$ times as large and with $n$ times the bandwidth, by reading and writing in parallel to all the base disks

RAID: use $n$ base disks of the same size to implement a single disk $n$ - 1 times as large and with $n$-1 times the bandwidth, but with much greater availability, by using the $n$th disk to store the exclusive-or of the others. Then if one disk fails, you can reconstruct its contents from the others.

Snapshots: use 'copy-on-write' to implement an ordinary disk and some number of read-only 'snapshots' of its previous state.

\section{Buffered files}

We need to make changes to the File spec if we want the option to implement it using buffered disks without doing too many syncs. One possibility is do a bdisk. sync at the end of each write. This spec is not what most systems implement, however, because it's too slow. Instead, they implement a version of File with the following additions. This version allows the data to revert to any previous state since the last Sync. The additions are very much like those we made to Disk to get BDisk. For simplicity, we don't change oldDirs for operations other than write and setSize (well, except for truncation); real systems differ in how much they buffer the other operations. 
MODULE File EXPORT $\ldots$, Sync $=$

TYPE ...

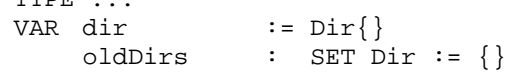

...

APROC Write (pn, $x$, byte $)=<\operatorname{VAR}$ fo $:=\operatorname{dir}(p n)$

$\operatorname{dir}(\mathrm{pn}):=\operatorname{NewFile}(\mathrm{f} 0, \mathrm{x}$, data)

oldDirs $\backslash /:=\{i \mid i<$ data.size

$\operatorname{dir}\{p n \rightarrow \operatorname{NewFile}(f 0, x, \operatorname{data.sub}(0, i)))\}>>$

APROC Sync ()$=\langle<$ OldDirs $:=\{\}>>$

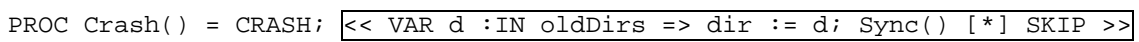

END File

Henceforth we will use File to refer to the modified module. Since we are not giving an implementation, we leave out Forget for simplicity.

Many file systems do their own caching and buffering. They usually loosen this spec so that a crash resets each file to some previous state, but does not necessarily reset the entire system to a previous state. (Actually, of course, real file systems usually don't have a spec, and it is often very difficult to find out what they can actually do after a crash.)

MODULE File2 EXPORT $\ldots$, Sync $=$

TYPE ...

OldFiles $\quad=$ PN $\rightarrow$ SET F

VAR dir

$$
:=\operatorname{Dir}\{\}
$$

oldFiles $\quad:=$ OldFiles $\{* \rightarrow\}$

$\cdots$

APROC Write (pn, $x$, byte $)=<\operatorname{VAR}$ fo $:=\operatorname{dir}(p n)$

$\operatorname{dir}(\mathrm{pn}):=\operatorname{NewFile}(\mathrm{f} 0, \mathrm{x}, \mathrm{data})$

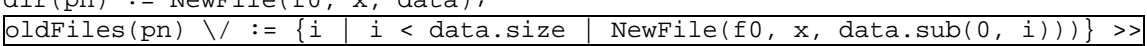

APROC Sync ()$=\langle<$ OldFiles:=OldFiles $\{*->\{\}\}>$

$\operatorname{PROC} \operatorname{Crash}()=$

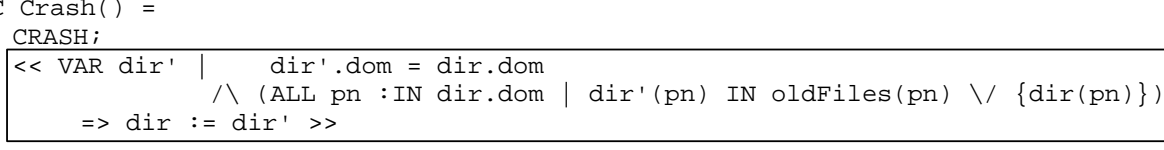

END File

A picky point about Spec: A function constructor like ( $\backslash \mathrm{pn} \mid\{\operatorname{dir}(\mathrm{pn})\})$ is no good as a value for oldFiles, because the value of the global variable dir in that constructor is not captured when the constructor is evaluated. Instead, this function uses the value of dir when it is invoked. This is a little weird, but it is usually very convenient. Here it is a pain; we avoid the problem by using a local variable d whose value is captured when the constructor is evaluated in SnapshotDir.
A still weaker spec allows dir to revert to a state in which any subset of the byte writes has been done, except that the files still have to be sequences. By analogy with unordered BDisk, we change the assignment to oldFiles in write.

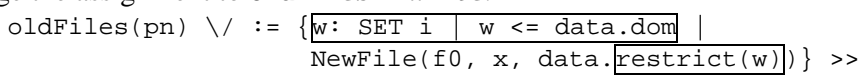

\section{Implementing files}

The main issue is how to represent the bytes of the file on the disk so that large reads and writes will be fast, and so that the file will still be there after a crash. The former requires using contiguous disk blocks to represent the file as much as possible. The latter requires a representation for Dir that can be changed atomically. In other words, the file system state has type $P N \rightarrow S E Q$ Byte, and we have to find a disk representation for the SEQ Byte that is efficient, and one for the function that is robust. This section addresses the first problem.

The simplest approach is to represent a file by a sequence of disk blocks, and to keep an index that is a sequence of the DA's of these blocks. Just doing this naively, we have
TYPE F
$=[$ das: $\operatorname{SEQ} D A$, size: $N]$
$\%$ Contents and size in bytes

The abstraction function to the spec says that the file is the first $\mathrm{f}$. size bytes in the disk blocks pointed to by c. Writing this as though both File and its implementation FImplo had the file $f$ as the state, we get

File.f $=(+:($ FImpl0.f.das * disk.disk $)) . \operatorname{seg}(0$, FImplo.f.size

or, using the disk.read method rather than the state of disk directly

File.f $=(+:\{d a: I N$ FImplo.f.das || disk.read(E\{da, 1\}) $\}) . \operatorname{seg}(0$, FImpl0.f.size $)$

But actually the state of File is dir, so we should have the same state for FImpl (with the different representation for $\mathrm{F}$, of course), and

File.dir $=($ LAMBDA $(\mathrm{pn})->$ File.F $=$

VAR $f:=$ FImpl0.dir $(p n)$

$\%$ fails if dir is undefined at pn

$\operatorname{RET}(+$ : (f.das * disk.disk)).seg (0, f.size)

We need an invariant that says the blocks of each file have enough space for the data.

\% INVARIANT ( ALL $f$ :IN dir.rng $\mid$ f.das.size * DBSize >=f.size)

Then it's easy to see how to implement read:

$\mathrm{PROC} \operatorname{read}(\mathrm{pn}, \mathrm{x}, \mathrm{i})=$

VAR $f:=\operatorname{dir}(\mathrm{pn})$

diskData $:=+:($ da $: \operatorname{IN~}$ f.das $\mid$ disk.read(E\{da, 1\})\},

fileData $:=$ diskData.seg $(0, f . s i z e)$

RET fileData.seg(x, i)

To implement write we need a way to allocate free DAs; we defer this to the next section. 
There are two problems with using this representation directly:

1. The index takes up quite a lot of space (with 4 byte DA's and DBSize $=1$ Kbyte it takes $.4 \%$ of the disk). Since RAM costs about 50 times as much as disk, keeping it all in RAM will add about $20 \%$ to the cost of the disk, which is a significant dollar cost. On the other hand, if the index is not in RAM it will take two disk accesses to read from a random file address, which is significant performance cost.

2. The index is of variable length with no small upper bound, so representing the index on the disk is not trivial either.

To solve the first problem, store Disk.E's in the index rather than DA's. A single extent can represent lots of disk blocks, so the total size of the index can be much less. Following this idea, we would represent the file by a sequence of Disk.E's, stored in a single disk block if it isn't too big or in a file otherwise. This recursion obviously terminates. It has the drawback that random access to the file might become slow if there are many extents, because it's necessary to search them linearly to find the extent that contains byte $\mathrm{x}$ of the file.

To solve the second problem, use some kind of tree structure to represent the index. In standard Unix file systems, for example, the index is a structure called an inode that contains:

a sequence of $10 \mathrm{DA}$ 's (enough for a $10 \mathrm{~KB}$ file, which is well above the median file size), followed by

the DA of an indirect DB that holds DBSize $/ 4=250$ or so DA's (enough for a $250 \mathrm{~KB}$ file), followed by

the DA of a second-level indirect block that holds the DA's of 250 indirect blocks and hence points to $250^{2}=62500 \mathrm{DA}$ 's (enough for a $62 \mathrm{MB}$ file),

and so forth. The third level can address an 16 GB file, which is enough for today's systems.

Thus the inode itself has room for 13 DA's. These systems duck the first problem; their extents are always a single disk block.

We give an implementation that incorporates both extents and trees, representing a file by a generalized extent that is a tree of extents. The leaves of the tree are basic extents Disk.E, that is, references to contiguous sequences of disk blocks, which are the units of $i / o$ for disk. read and disk.write. The purpose of such a general extent is simply to define a sequence of disk addresses, and the E. das method computes this sequence so that we can use it in invariants and abstraction functions. The tree structure is there so that the sequence can be stored and modified more efficiently.

An extent that contains a sequence of basic extents is called a linear extent. To do fast $\mathrm{i} / \mathrm{o}$ operations, we need a linear extent which includes just the blocks to be read or written, grouped into the largest possible basic extents so that disk. read and disk. write can work efficiently. Flatten computes such a linear extent from a general extent; the spec for Flatten given below flattens the entire extent for the file and then extracts the smallest segment that contains all the blocks that need to be touched.

Read and Write just call Flatten to get the relevant linear extent and then call disk.read and disk.write on the basic extents; Write may extend the file first, and it may have to read the first and last blocks of the linear extent if the data being written does not fill them, since the disk can only write entire blocks. Extending or truncating a file is more complex, because it requires changing the extent, and also because it requires allocation. Allocation is described in the next section. Changing the extent requires changing the tree.

The tree itself must be represented in disk blocks; methods inspired by B-trees can be used to change it while keeping it balanced. Our implementation shows how to extract information from the tree, but not how it is represented in disk blocks or how it is changed. In standard Unix file systems, changing the tree is fairly simple because a basic extent is always a single disk block in the multi-level indirect block scheme described above.

We give the abstraction function to the simple implementation above. It just says that the das of a file are the ones you get from Flatten.

The code below makes heavy use of function composition to apply some function to each element of a sequence: $s * f$ is $\{f(s(0)), \ldots, f(s(s . s i z e-1))\}$. If $f$ yields an integer or a sequence, the combination $+:(s * f)$ adds up or concatenates all the $f(s(i))$.

\section{MODULE FSImpl $=$}

TYPE N

CONST DBSize
$=\mathrm{Nat}$

$=[C:$ (Disk DA + SE) r size: $]$ SUCHTHAT ( $(\backslash \mathrm{e} \mid$ Size $(e)=$ e.size $)$ WITH $\{$ das:=ETODAs, le:=ETOLE

$=$ E SUCHTHAT $(\backslash e \mid$ e.c IS Disk.DA) $=$ E SUCHTHAT (\e| e.c IS SEQ BE) WITH $\{"+":=$ Cat $\}$

$=\mathrm{SEQ} \mathrm{E}$

$=$ File.x

$=[e$, size: $x]$

$=$ File.PN

$:=1024$

VAR dir : File.PN $\rightarrow F:=\{$

$\therefore$ ABSTRACTION FUNCTION File.dir $=(\operatorname{LAMBDA}(\mathrm{pn})->$ File.F $=$ dir $! \mathrm{pn}=$

$\%$ The file is the first $f$.size bytes in the disk blocks of the extent $f . e$

VAR $f:=\operatorname{dir}(p n)$,

d:= + $:\{$ be :IN Flatten(f.e, 0, f.e.size).c | | disk.read(be) $\}$

RET d.seg(0,f.size) )

$\therefore$ ABSTRACTION FUNCTION FImplo.dir $=(\operatorname{LAMBDA}(\mathrm{pn})->$ FImplO.F $=$

$\operatorname{VAR} f:=\operatorname{dir}(\mathrm{pn}) \mid \operatorname{RET}\{$ be $: \operatorname{IN}$ Flatten(f.e, o, f.e.size).c $\mid$ be.c

FUNC Size $(e) \rightarrow$ Int $=$ RET ( e IS BE $\Rightarrow$ e, size $[*]+:(e, c *$ Size $)$ )

$\%$ \# of DA's reachable from $\mathrm{e}$. Should be equal to e. size. 
FUNC Cat $(l e 1, l e 2) \rightarrow \operatorname{LE}=$

$\%$ The "+" method of LE. Merge e1 and e2 if possible.

IF $e 1=\{\} \Rightarrow$ RET le2

[] $\mathrm{e} 2=\{\} \Rightarrow$ RET le1

[] VAR e1 := le1.c.last, e2 := le2.c.head, se |

IF $\mathrm{e} 1 . \mathrm{c}+\mathrm{e} 1 . \mathrm{size}=\mathrm{e} 2 \cdot \mathrm{c} \Rightarrow$

se := le1.c.reml $+\operatorname{SE}\{E\{e 1 . c, e 1 . s i z e+e 2 . s i z e\}\}+$ le2.c.tail $\left[{ }^{*}\right]$ se $:=1 \mathrm{e} 1 . \mathrm{c}+1 \mathrm{e} 2 . \mathrm{c}$

$\mathrm{FI}$;

FI

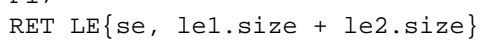

FUNC Flatten(e, start: N, size: N) $\rightarrow$ LE = VAR leo :=e.le, le1, le2, le3 |

$\%$ The result le is such that le.das $=$ e.das.seg (start, size);

$\%$ This is fewer than size DA's if e gets used up.

\% It's empty if start $>=$ e.size.

$\%$ This is not a practical implementation; see below. $l e 0=1 e 1+l e 2+l e 3$

八le1.size $=\{$ start, e.size $\}$ min

$\wedge$ le2.size $=\{$ size, $\{$ e.size - start, 0$\} \cdot \max \} \cdot \min$

$\Rightarrow$ RET le2

$\ldots$

END FSImpl

This version of Flatten is not very practical; in fact, it is more like a spec than an implementation. A practical one, given below, searches the tree of extents sequentially, taking the largest possible jumps, until it finds the extent that contains the startth DA. Then it collects extents until it has gotten size DA's. Note that because each e.size gives the total number of DA's in e, Flatten only needs time log (e.size) to find the first extent it wants, provided the tree is balanced. This is a standard trick for doing efficient operations on trees: summarize the important properties of each subtree in its root node. A further refinement (which we omit) is to store cumulative sizes in an SE so that we can find the point we want with a binary search rather than the linear search in the Do loop below; we did this in the editor buffer example of handout 3 .

FUNC Flatten (e, start: $N$, size: N) $\rightarrow$ LE $=$

VAR $z:=\{$ size, \{e.size - start, 0$\}$. max $\}$.min

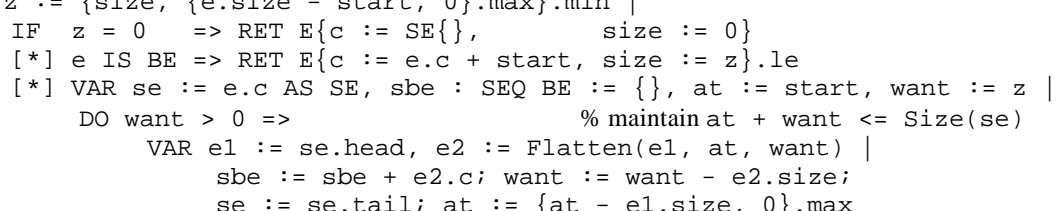




\section{Encoding and decoding}

To store complicated values on the disk, such as the function that constitutes a directory, we need to encode them into a byte sequence, since Disk. Data is SEQ Byte. (We also need encoding to send values in messages, an important operation later in the course.) It's convenient to do this with a pair of functions for each type, called Encode and Decode, which turn a value of the type into a byte sequence and recover the value from the sequence. We package them up into an EncDec pair.

TYPE D

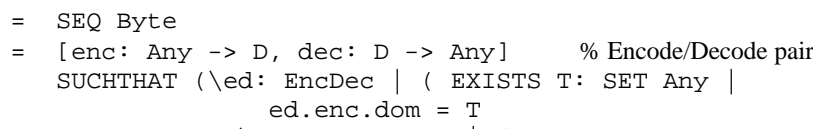

Other names for 'encode' are 'serialize', 'pickle', and 'marshal'.

A particular EncDec works only on values of a single type (represented by the set $\mathrm{T}$ in the SUCHTHAт, since you can't quantify over types in Spec). This means that enc is defined exactly on values of that type, and dec is the inverse of enc so that the process of encoding and then decoding does not lose information. We do not assume that enc is the inverse of dec, since there may be many byte sequences that decode to the same value; for example, if the value is a set, it would be pointless and perhaps costly to insist on a canonical ordering of the encoding. In this course we will generally assume that every type has methods enc and dec that form an EncDec pair.

A type that has other types as its components can have its EncDec defined in an obvious way in terms of the EncDec's of the component types. For example, a SEQ T can be encoded as a sequence of encoded T's, provided the decoding is unambiguous. A function $T \rightarrow U$ can be encoded as a set or sequence of encoded $(T, U)$ pairs.

A directory is one example of a situation in which we need to encode a sequence of values into a sequence of bytes. A log is another example of this, discussed below, and a stream of messages is a third. It's necessary to be able to parse the encoded byte sequence unambiguously and recover the original values. We can express this idea precisely by saying that a parse is an EncDec sequence, a language is a set of parses, and the language is unambiguous if for every byte sequence $d$ the language has at most one parse that can completely decode $d$.

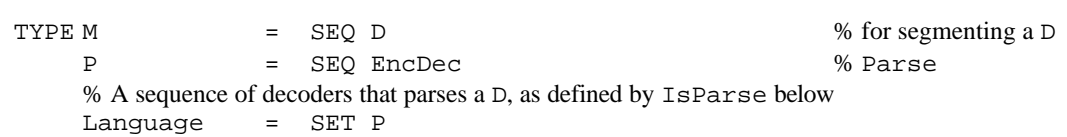

Of course ambiguity is not decidable in general. The standard way to get an unambiguous language for encodings is to use type-length-value (TLV) encoding, in which the result $d$ of enc (x) starts with some sort of encoding of x's type, followed by an encoding of d's own length, followed by a $D$ that contains the rest of the information the decoder needs to recover $x$.

FUNC IsTLV(ed: EncDec) $\rightarrow$ Bool =

$$
\begin{gathered}
\text { RET (ALL } x \text { : IN ed.enc.dom } \mid \text { (EXISTS } d 1, d 2, d 3 \mid \\
\text { ed.enc }(x)=d 1+d 2+d 3 八 \text { EncodeType }(x)=d 1 \\
\text { 八 (ed.enc }(x) \text {. size).enc }=d 2 \text { )) }
\end{gathered}
$$

In many applications there is a grammar that determines each type unambiguously from the preceding values, and in this case the types can be omitted. For instance, if the sequence is the encoding of a SEQ T, then it's known that all the types are T. If the length is determined from the type it can be omitted too, but this is done less often, since keeping the length means that the decoder can reliably skip over parts of the encoded sequence that it doesn't understand. If desired, the encodings of different types can make different choices about what to omit.

There is an international standard called ASN-1 (for Abstract Syntax Notation) that defines a way of writing a grammar for a language and deriving the EncDec pairs automatically from the grammar. Like most such standards, it is rather complicated and often yields somewhat inefficient encodings, but it is fairly widely used.

Another standard way to get an unambiguous language is to encode into S-expressions, in which the encoding of each value is delimited by parentheses, and the type, unless it can be omitted, is given by the first symbol in the S-expression. A variation on this scheme which is popular for Internet Email and Web protocols, is to have a 'header' of the form

attribute1: value1

attribute2: value2

with various fairly ad-hoc rules for delimiting the values that are derived from early conventions for the human-readable headers of Email messages.

In both TLV and S-expression encodings, decoding depends on knowing exactly where the byte sequence starts. This is not a problem for D's coming from a file system, but it is a serious problem for D's coming from a wire or byte stream, since the wire produces a continuous stream of voltages, bits, bytes, or whatever. The process of delimiting a stream of symbols into D's that can be decoded is called framing; we will discuss it later in connection with networks.

\section{Directories}

Recall that a Dir is just a PN $\rightarrow F$. We have seen various ways to represent $F$. The simplest implementation relies on an EncDec for an entire Dir. It represents a Dir as a file containing enc of the $\mathrm{PN} \rightarrow \mathrm{F}$ map as a set of ordered pairs.

There are two problems with this scheme:

- Lookup in a large Dir will be slow, since it requires decoding the whole Dir. This can be fixed by using a hash table or B-tree. Updating the Dir can still be done as in the simple scheme, but this will also be slow. Incremental update is possible, if more complex; it also has atomicity issues. 
- If we can't do an atomic file write, then when updating a directory we are in danger of scrambling it if there is a crash during the write. There are various ways to solve this problem. The most general and practical way is to use the transactions explained in the next section.

It is very common to implement directories with an extra level of indirection called an 'inode', so that we have

$$
\begin{array}{ll}
\text { TYPE INO } & =\text { Int } \\
\text { Dir } & =\text { PN } \rightarrow \text { INo } \\
\text { INOMap } & =\text { INo } \rightarrow \text { F } \\
\text { VAR dir } & : \text { Dir }:=\{\} \\
\text { inodes } & : \text { INoMap }:=\{\}
\end{array}
$$

$\%$ Inode Number

You can see that inodes is just like a directory except that the names are INo's instead of PN's. There are three advantages:

Because INo's are integers, they are cheaper to store and manipulate. It's customary to provide an Open operation to turn a PN into an INo (usually through yet another level of indirection called a 'file descriptor'), and then use the INo as the argument of Read and Write.

Because INo's are integers, if $\mathrm{F}$ is fixed-size (as in the Unix example discussed earlier, for instance) then inodes can be represented as an array on the disk that is just indexed by the INo.

The enforced level of indirection means that file names automatically get the semantics of pointers or memory addresses: two of them can point to the same file variable.

The third advantage can be extended by extending the definition of Dir so that the value of a PN can be another PN, usually called a "symbolic link".

TYPEDir $\quad=$ PN $\rightarrow($ INO + PN $)$

\section{Transactions}

We have seen several examples of a general problem: to give a spec for what happens after a crash that is acceptable to the client, and an implementation that satisfies the spec even though it has only small atomic actions at its disposal. In writing to a file, in maintaining allocation information, and in updating a directory, we wanted to make a possibly large state change atomic in the face of crashes during its execution, even though we can only write a single disk block atomically.

The general technique for dealing with this problem is called transactions. General transactions make large state changes atomic in the face of arbitrary concurrency as well as crashes; we will discuss this later. For now we confine ourselves to 'sequential transactions', which only take care of crashes. The idea is to conceal the effects of a crash entirely within the transaction abstraction, so that its clients can program in a crash-free world.
The implementation of sequential transactions is based on the very general idea of a deterministic state machine that has inputs called actions and makes a deterministic transition for every input it sees. The essential observation is that:

If two instances of a deterministic state machine start in the same state and see the
same inputs, they will make the same transitions and end up in the same state.

This means that if we record the sequence of inputs, we can replay it after a crash and get to the same state that we reached before the crash. Of course this only works if we start in the same state, or if the state machine has an 'idempotency' property that allows us to repeat the inputs. More on this below.

Here is the spec for sequential transactions. There's a state that is queried and updated (read and written) by actions. We keep a stable version ss and a volatile version vs. Updates act on the volatile version, which is reset to the stable version after a crash. A 'commit' action atomically sets the stable state to the current volatile state.

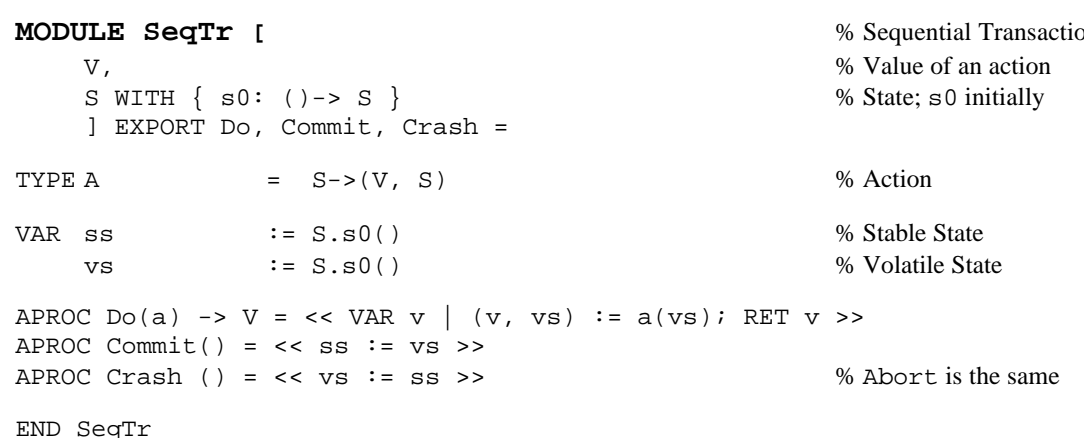

In other words, you can do a whole series of actions to the volatile state vs, followed by a Commit. Think of the actions as reads and writes, or queries and updates. If there's a crash before the Commit, the state reverts to what it was initially. If there's a crash after the commit, the state reverts to what it was at the time of the commit. An action is just a function from an initial state to a final state and a result value.

There are many implementation techniques for transactions. Here is the simplest. It breaks each action down into a sequence of updates, each one of which can be done atomically; the most common example of an atomic update is a write of a single disk block. The updates also mus have an 'idempotency' property discussed later. Given a sequence of Do's, each applying an action, the implementation concatenates the update sequences for the actions in a volatile log that is a representation of the actions. Commit writes this log atomically to a stable log. Once the stable log is written, Redo applies the volatile log to the stable state and erases both logs. Crash resets the volatile to the stable $\log$ and then applies the $\log$ to the stable state to recover the volatile state. It then uses Redo to update the stable state and erase the logs. Note that we give $\mathrm{S}$ a "+" method $s+1$ that applies a $\log$ to a state. 
This scheme reduces the problem of implementing arbitrary changes atomically to the problem of atomically writing an arbitrary amount of stuff to a log. This is easier but still not trivial to do efficiently; we discuss it at the end of the section.

\section{MODULE LogRecovery [}

SO WITH $\{$ so: () $\rightarrow$ so $\}$

] EXPORT Do, Commit, Crash $=$

$\begin{aligned} \text { TYPE A } & =S->(\mathrm{V}, \mathrm{S}) \\ \mathrm{U} & =\mathrm{S}->\mathrm{S} \\ \mathrm{L} & =\mathrm{SEQU} \\ \mathrm{S} & =\mathrm{SO} \mathrm{WITH}\{"+":=\text { DoLog }\} \\ \text { VAR SS } & :=\mathrm{S} . \mathrm{SO}() \\ \mathrm{VS} & :=\mathrm{S} 0() \\ \mathrm{SI} & :=\mathrm{L}\{\} \\ \mathrm{VI} & :=\mathrm{L}\{\}\end{aligned}$

ABSTRACTION to SegTr SeqTr.ss $=$ ss + sl SeqTr.ss $=$ Ss
SeqTr.vs $=$ vs

$\therefore$ INVARIANT $\mathrm{vS}=\mathrm{sS}+\mathrm{vl}$

FUNC DoLog $(s, 1) \rightarrow S=$

$\%$ Apply the updates in 1 to the state $s$.

$\left.l=\{\} \Rightarrow \operatorname{RET} s{ }^{*}\right] \operatorname{RET} \operatorname{DoLog}((1$. head) (s), l.tail))

APROC Do (a) $\rightarrow \mathrm{V}=$

$\%$ Find an $I$ (a sequence of updates) that has the same effect as a on the current state.

$\ll \operatorname{VAR} v, l \mid(\mathrm{v}, \mathrm{vs}+1)=\mathrm{a}(\mathrm{vs}) \Rightarrow$

$$
\mathrm{vl}:=\mathrm{vl}+1 ; \mathrm{vs}:=\mathrm{vs}+1 ; \operatorname{RET} \mathrm{v}>>
$$

PROC Commit () = <<sl := vl >>; Redo()

PROC Redo () =

$\%$ replay vi, then clear $\mathrm{s} I$

DO vl \# \{\}$=><<$ ss :=ss + vl.head; vl :=vl.tail $>$ OD; $<<$ sl $:=\{\}>>$

$\operatorname{PROC} \operatorname{Crash}()=$

CRASH;

$<<\mathrm{vl}:=\{\} ;$ vs $:=\mathrm{S} . \mathrm{s} 0()>>$

$<<\mathrm{vl}:=\mathrm{sl} ; \mathrm{vs}:=\mathrm{ss}+\mathrm{vl}>>_{i}$

Redo ()

$\%$ crash erases vs, vi

$\%$ recovery restores them

$\%$ and repeats the Redo; this is optional

END LogRecovery

For this redo crash recovery to work, I must have the property that repeatedly applying prefixes of it, followed by the whole thing, has the same effect as applying the whole thing. For example, suppose $1=L\{a, b, c, d, e\}$. Then $L\{a, b, c, a, a, a, b, c, d, a, b, a, b, c, d, e, a, a, b, c, d, e\}$ must have the same effect as 1 itself; here we have grouped the prefixes together for clarity. We need this property because a crash can happen while Redo is running; the crash reapplies the whole log and runs Redo again. Another crash can happen while the second Redo is running, and so forth.
This 'hiccup' property follows from 'log idempotence':

$$
s+1+1=s+1
$$

From this we get (recall that $<$ is the 'prefix' predicate for sequences).

$$
k<1=0(s+k+1=s+1)
$$

because $k<1$ implies there is a $l^{\prime}$ such that $k+l^{\prime}=1$, and hence $s+k+l=s+k+\left(k+1 l^{\prime}\right)=(s+k+k)+l^{\prime}$

$=(\mathrm{s}+\mathrm{k})+\mathrm{l}^{\prime}=\mathrm{s}+\left(\mathrm{k}+\mathrm{l}^{\prime}\right)=\mathrm{s}+\mathrm{l}$

From (2) we get the property we want:

IsHiccups $(k, 1)=\Rightarrow(s+k+1=s+1)$

where

FUNC IsHiccups $(k, 1) \rightarrow$ Bool $=$

$\% \mathrm{k}$ is a sequence of attempts to complete

$\operatorname{RET} k=\{\}$

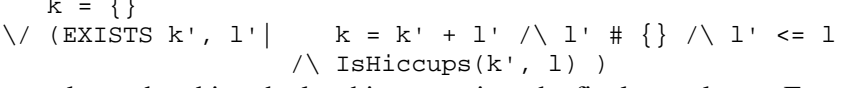

because we can keep absorbing the last hiccup 1 ' into the final complete 1. For example, taking some liberties with the notation for sequences:

$$
\begin{aligned}
& \text { abcaaabcdababcdeaabcde } \\
= & \text { abcaaabcdababcde }+(a+\text { abcde }) \\
= & \text { abcaaabcdababcde }+a b c d e \\
= & a b c a a a b c d a b+(a b c d e+\text { abcde }) \\
= & \text { abcaaabcdab }+a b c d e \\
= & a b c a a a b c d+(a b+a b c d e) \\
= & \text { abcaaabcd }+ \text { abcde }
\end{aligned}
$$

and so forth.

To prove (3), observe that

$\operatorname{IsHiccups}(\mathrm{k}, 1) 八 \mathrm{k} \#\{\}==\mathrm{k}^{\prime}=\mathrm{k}^{\prime}+\mathrm{l}^{\prime}$ 八 $\mathrm{l}^{\prime}<=1$ 八IsHiccups(k', l). Hence

$\mathrm{s}+\mathrm{k}+1=\left(\mathrm{s}+\mathrm{k}^{\prime}\right)+1{ }^{\prime}+1=\mathrm{s}+\mathrm{k}^{\prime}+1$

by (2)

and $k^{\prime}<k$. But we have IsHiccups $\left(k^{\prime}, 1\right)$, so we can proceed by induction until $k^{\prime}=\{\}$ and we have the desired result.

We can get log idempotence if the U's commute and are idempotent (that is, $u \star u=u$ ), or if they are all writes. More generally, for arbitrary U's we can attach a UID to each U and record it in $\mathrm{S}$ when the $\mathrm{U}$ is applied, so we can tell that it shouldn't be applied again. Calling the original state $\mathrm{SS}$, and defining a meaning method that turns a $\mathrm{U}$ record into a function, we have TYPE

S $\quad=[S S$, tags: SET UID $]$

$=[$ uu: SS->SS, tag: UID $]$ WITH \{ meaning:=Meaning $\}$

FUNC Meaning $(u, s)->S=$

$$
\text { u.tag IN s.tags } \Rightarrow \text { RET } s
$$

$\%$ u already done

[*] RET S\{ (u.uu) (s.ss), s.tags + \{u.tag $\}$

If all the U's in 1 have different tags, we get log idempotence. The tags make U's 'testable' in the jargon of transaction processing; after a crash we can test to find out whether a $\mathrm{U}$ has been done or not. In the standard database implementation each $\mathrm{U}$ works on one disk page, the tag is the 'log 
sequence number', the index of the update in the log, and the update writes the tag on the disk page.

\section{Writing the log atomically}

There is still an atomicity problem in this implementation: Commit atomically does $<$ sl : : vl >>, and the logs can be large. A simple way to use a disk to implement a log that requires this assignment of arbitrary-sized sequences is to keep the size of sl in a separate disk block, and to write all the data first, then do a Sync if necessary, and finally write the new size. Since $\mathrm{sl}$ is always empty before this assignment, in this representation it will remain empty until the single Disk.write that sets its size. This is a rather wasteful implementation, since it does an extra disk write.

A more efficient implementation writes a 'commit record' at the end of the log, and treats the log as empty unless the commit record is present. Now it's only necessary to ensure that the log can never be mis-parsed if a crash happens while it's being written. An easy way to accomplish this is to write a distinctive 'erased value into each disk block that may become part of the log, but this means that for every disk write to a log block, there will be another write to erase it. To avoid this cost we can use a ring buffer of disk blocks for the log and a sequence number that increments each time the ring buffer wraps around; then a block is 'erased' if its sequence number is not the current one. There's still a cost to initialize the sequence numbers, but it's only paid once. With careful implementation, a single bit of sequence number is enough.

In some applications it's inconvenient to make room in the data stream for a sequence number every DBsize bytes. To get around this, use a 'displaced' representation for the log, in which the first data bit of each block is removed from its normal position to make room for the one bit sequence number. The displaced bits are written into their own disk blocks at convenient intervals.

Another approach is to compute a strong checksum for the log contents, write it at the end after all the other blocks are known to be on the disk, and treat the log as empty unless a correct checksum is present. With a good $n$-bit checksum, the probability of mis-parsing is $2^{-n}$.

\section{Redundancy}

A disk has many blocks. We would like some assurance that the failure of a single block will not damage a large part of the file system. To get such assurance we must record some critical parts of the representation redundantly, so that they can be recovered even after a failure.

The simplest way to get this effect is to record everything redundantly. This gives us more: a single failure won't damage any part of the file system. Unfortunately, it is expensive. In current systems this is usually done at the disk abstraction, and is called mirroring or shadowing the disk.

The alternative is to record redundantly only the information whose loss can damage more than one file: extent, allocation, and directory information.

\section{Another approach is to}

do all writes to a $\log$,

keep a copy of the log for a long time (by writing it to tape, usually), and

checkpoint the state of the file system occasionally.

Then the current state can be recovered by restoring the checkpoint and replaying the log from the moment of the checkpoint. This method is usually used in large database systems, but not in any file systems that I know of.

We will discuss these methods in more detail near the end of the course

\section{Copying File Systems}

The file system described in FSImpl above separates the process of adding DB's to the representation of a file from the process of writing data into the file. A copying file system (CFS) combines these two processes into one. It is called a 'log-structured' file system in the literature ${ }^{1}$ but as we shall see, the log is not the main idea. A CFS is based on three ideas:

- Use a generational copying garbage collector (called a cleaner) to reclaim DB's that are no longer reachable and keep all the free space in a single (logically) contiguous region, so that there is no need for a bit table or free list to keep track of free space.

- Do all writes sequentially at one end of this region, so that existing data is never overwritten and new data is sequential.

- Log and cache updates to metadata (the index and directory) so that the metadata doesn't have to be rewritten too often.

A CFS is a very interesting example of the subtle interplay among the ideas of sequential writing, copying garbage collection, and logging. This section describes the essentials of a CFS in detail and discusses more briefly a number of refinements and practical considerations. It will repay careful study.

Here is a picture of a disk organized for a CFS: $\mathrm{abc}==$ de $\mathrm{fgh}====\mathrm{i} j \mathrm{kl}=\mathrm{m}=$ nopqrs

In this picture letters denote reachable blocks, ='s denote unreachable blocks that are not part of the free space, and -'s denote free blocks (contiguous on the disk viewed as a ring buffer). After the cleaner copies blocks a-e the picture is

$$
\text { --------fgh }====i j \mathrm{kl}=\mathrm{m}=\text { nopqr sabcde- }
$$

because the data a-e has been copied to free space and the blocks that used to hold a-e are free, together with the two unreachable blocks which were not copied. Then after blocks $g$ and $j$ are overwritten with new values $\mathrm{G}$ and $\mathrm{J}$, the picture is

$$
------\mathrm{f}=\mathrm{h}====\mathrm{i}=\mathrm{k} \mathrm{l}=\mathrm{m}=\text { nopqr sabcdeG } \mathrm{J}
$$

${ }^{1}$ M. Rosenblum and J. Osterhout, The design and implementation of a log-structured file system, $A C M$ Transactions on Computer Systems, 10, 1, Feb. 1992, pp 26-52. 
The new data $\mathrm{G}$ and $\mathrm{J}$ has been written into free space, and the blocks that used to hold $g$ and $j$ are now unreachable. After the cleaner runs to completion the picture is

------------------nopqrsabcdeGJ fhiklm----

Pros and cons

A CFS has two main advantages:

- All writing is done sequentially; as we know, sequential writes are much faster than random writes. We have a good technique for making disk reads faster: caching. As main memory caches get bigger, more reads hit in the cache and disks spend more of their time writing, so we need a technique to make writes faster.

- The cleaner can copy reachable blocks to anywhere, not just to the standard free space region, and can do so without interfering with normal operation of the system. In particular, it can copy reachable blocks to tape for backup, or to a different disk drive that is faster, cheaper, less full, or otherwise more suitable as a home for the data.

There are some secondary advantages. Since the writes are sequential, they are not tied to disk blocks, so it's easy to write items of various different sizes without worrying about how they are packed into DB's. Furthermore, it's easy to compress the sequential stream as it's being written ${ }^{2}$, and if the disk is a RAID you never have to read any blocks to recompute the parity. Finally, there is no bit table or free list of disk blocks to maintain.

There is also one major drawback: unless large amounts of data in the same file are written sequentially, a file will tend to have lots of small extents, which can cause the problems discussed on page 13. In Unix file systems most files are written all at once, but this is certainly not true for databases. Ways of alleviating this drawback are the subject of current research. The cost of the cleaner is also a potential problem, but in practice the cost of the cleaner seems to be small compared to the time saved by sequential writes.

\section{Updating metadata}

For the CFS to work, it must update the index that points to the DB's containing the file data on every write and every copy done by the cleaner, not just when the file is extended. And in order to keep the writing sequential, we must handle the new index information just like the file data, writing it into the free space instead of overwriting it. This means that the directory too must be updated, since it points to the index; we write it into free space as well. Only the root of the entire file system is written in a fixed location; this root says where to find the directory

You might think that all this rewriting of the metadata is too expensive, since a single write to a file block, whether existing or new, now triggers three additional writes of metadata: for the index (if it doesn't fit in the directory), the directory, and the root. Previously none of these writes was needed for an existing block, and only the index write for a new block. However, the scheme for logging updates that we introduced to implement transactions can also handle this

${ }^{2}$ M. Burrows et al., On-line compression in a log-structured file system, Proc. 5th Conference on Architectural Support for Programming Languages and Operating Systems, Oct. 1992, pp 2-9. This does require some blocking so that the decompressor can obtain the initial state it needs. problem. The idea is to write the changes to the index into a log, and cache the updated index (or just the updates) only in main memory. An example of a logged change is "block 43 of file 'alpha' now has disk address 385672". Later (with any luck, after several changes to the same piece of the index) we write the index itself and log the consequent changes to the directory; again, we cache the updated directory. Still later we write the directory and log the changes to the root. We only write a piece of metadata when:

We run out of main memory space to cache changed metadata, or

The log gets so big (because of many writes) that recovery takes too long.

To recover we replay the active tail of the log, starting before the oldest logged change whose metadata hasn't been rewritten. This means that we must be able to read the log sequentially from that point. It's natural to write the log to free space along with everything else. While we are at it, we can also log other changes like renames.

Note that a CFS can use exactly the same directory and index data as an ordinary file system, and in fact exactly the same code for Read. To do this we must give up the added flexibility we can get from sequential writing, and write each DB of data into a DB on the disk. Several implementations have done this (but the simple implementation below does not).

The logged changes serve another purpose. Because a file can only be reached from a single directory entry (or inode), the cleaner need not trace the directory structure in order to find the reachable blocks. Instead, if the block at da was written as block $b$ of file $f$, it's sufficient to look at the file index and find out whether block $b$ of file $f$ is still at da. But the triple $(b, f, d a)$ is exactly the logged change. To take advantage of this we must keep the logged change as long as da remains reachable since the cleaner needs it (it's called 'segment summary' information in the literature). We don't need to replay it on recovery once its metadata is written out, however, and hence we need the sequential structure of the log only for the active tail.

Existing CFS's use the extra level of naming called inodes that is described on page 19. The inode numbers don't change during writing or copying, so the PN $\rightarrow$ INo directory doesn't change. The root points to index information for the inodes (called the 'inode map'), which points to inodes, which point to data blocks or, for large files, to indirect blocks which point to data blocks.

Segments

Running the cleaner is fairly expensive, since it has to read and write the disk. It's therefore important to get as much value out of it as possible, by cleaning lots of unreachable data instead of copying lots of data that is still reachable. To accomplish this, divide the disk into segments, large enough (say $1 \mathrm{MB}$ or $10 \mathrm{MB}$ ) that the time to seek to a new segment is much smaller than the time to read or write a whole segment. Clean each segment separately. Keep track of the amount of unreachable space in each segment, and clean a segment when (unreachable space) * (age of data) exceeds a threshold. Rosenblum and Osterhout explain this rule, which is similar in 
spirit to what a generational garbage collector ${ }^{3}$ does; the goal is to recover as much free space as possible, without allowing too much unreachable space to pile up in old segments.

Now the free space isn't physically contiguous, so we must somehow link the segments in the active tail together. We also need a table that keeps track for each segment of whether it is free, and if not, what its unreachable space and age are; this is cheap because segments are so large.

\section{Backup}

As we mentioned earlier, one of the major advantages of a CFS is that it is easier to back up. There are several reasons for this.

1. You can take a snapshot just by stopping the cleaner from freeing cleaned segments, and then copy the root information and the log to the backup medium, recording the logged data backward from the end of the log.

2. This backup data structure allows a single file (or a small set of files) to be restored in one pass.

3. It's only necessary to copy the log back to the point at which the previous backup started.

4. The disks reads done by backup are sequential and therefore fast. This is an important issue when the file system occupies many terabytes. At the $10 \mathrm{MB} / \mathrm{s}$ peak transfer rate of the disk, it takes $10^{5}$ seconds, or a bit more than one day, to copy a terabyte. This means that a small number of disks and tapes running in parallel can do it in a fraction of a day. If the transfer rate is reduced to $1 \mathrm{MB} / \mathrm{s}$ by lots of seeks (which is what you get with random seeks if the average block size is $10 \mathrm{~KB}$ ), the copying time becomes 10 days, which is impractical.

5. If a large file is partially updated, only the updates will be logged and hence appear in the backup.

6. It's easy to merge several incremental backups to make a full backup.

To get these advantages, we have to retain the ordering of segments in the log even after recovery no longer needs it.

There have been several research implementations of CFS's, and at least one commercial one called Spiralog in Digital Equipment Corporation's (now Compaq's) VMS system. You can read a good deal about it at http://www.digital.com/info/DTJM00/.

${ }^{3}$ H. Lieberman and C. Hewitt, A real-time garbage collector based on the lifetimes of objects, Comm. ACM 26, 6 , June 1983, pp 419-429.

\section{A simple CFS implementation}

We give an implementation CopyingFs of a CFS that contains all the essential ideas (except for segments, and the rule for choosing which segment to clean), but simplifies the data structures for the sake of clarity. CopyingFs treats the disk as a root DB plus a ring buffer of bytes. Since writing is sequential this is practical; the only cost is that we may have to pad to the end of a $D B$ occasionally in order to do a Sync. A DA is therefore a byte address on the disk. We could dispense with the structure of disk blocks entirely in the representation of files, just write the data of each File. Write to the disk, and make a FSImpl.BE point directly to the resulting byte sequence on the disk. Instead, however, we will stick with tradition, take BE $=$ DA, and represent a file as a SEQ DA plus its size.

So the disk consists of a root page, a busy region, and a free region (as we have seen, in a real system both busy and free regions would be divided into segments); see the figure below. The busy region is a sequence of encoded Item's, where an Item is either a Dir or a Change to a DB in a file or to the Dir. The busy region starts at busy and ends just before free, which always points to the start of a disk block. We could write free into the root, but then making anything stable would require a (non-sequential) write of the root. Instead, the busy region ends with a recognizable endDB, put there by Sync, so that recovery can find the end of the busy region.

DirDA is the address of the latest directory on the disk. The part of the busy region after dirDA is the active tail of the log and contains the changes that need to be replayed during recovery to reconstruct the current directory; this arrangement ensures that we start the replay with a dir to which it makes sense to apply the changes that follow.

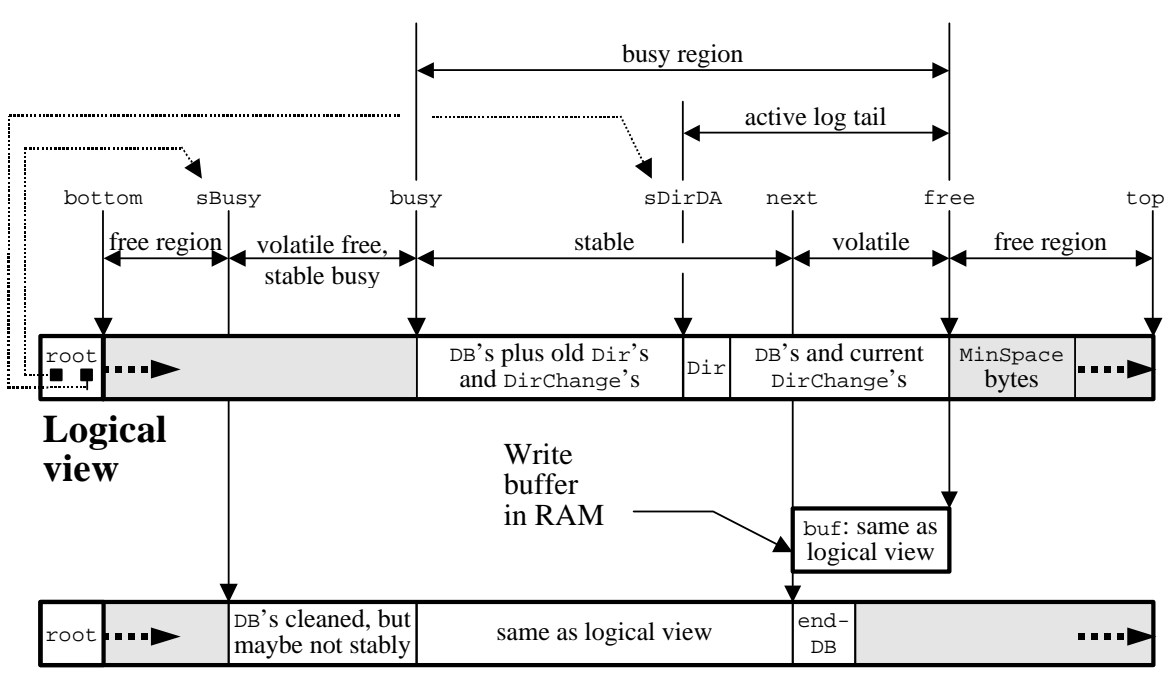

Disk 
This implementation does bytewise writes that are buffered in buf and flushed to the disk only by sync. Hence after a crash the state reverts to the state at the last sync. Without the replay done during recovery by ApplyLog, it would revert to the state the last time the root was written; be sure you understand why this is true.

We assume that a sequence of encoded Item's followed by an endDB can be decoded unambiguously. See the earlier discussion of writing logs atomically.

\section{Other simplifications:}

1. We store the $S E Q D A$ that points to the file $D B$ 's right in the directory. In real life it would be a tree, along one of the lines discussed in FSImpl, so that it can be searched and updated efficiently even when it is large. Only the top levels of the tree would be in the directory.

2. We keep the entire directory in main memory and write it all out as a single Item. In real life we would cache parts of it in memory and write out only the parts that are dirty (in other words, that contain changes).

3. We write a data block as part of the log entry for the change to the block, and make the DA's in the file representation point to these log entries. In real life the logged change information would be batched together (as 'segment summary information') and the data written separately, so that recovery and cleaning can read the changes efficiently without having to read the file data as well, and so that contiguous data blocks can be read with a single disk operation and no extra memory-to-memory copying.

4. We allocate space for data in write, though we buffer the data in buf rather than writing it immediately. In real life we might cache newly written data in the hope that another adjacent write will come along so that we can allocate contiguous space for both writes, thus reducing the number of extents and making a later sequential read faster.

5. Because we don't have segments, the cleaner always copies items starting at busy. In real life it would figure out which segments are most profitable to clean.

6. We run the cleaner only when we need space. In real life, it would run in the background to take advantage of times when the disk is idle, and to maintain a healthy amount of free space so that writes don't have to wait for the cleaner to run.

7. We treat WriteData and WriteRoot as atomic. In real life we would use one of the techniques for making log writes atomic that are described on page 23 .

8. We treat Init and Crash as atomic, mainly for convenience in writing invariants and abstraction functions.In real life they do several disk operations, so we have to lock out external invocations while they are running.

9. We ignore the possibility of errors.
MODULE COPYingFS EXPORTS PN, SYnc $=$

$\%$ implements File, uses Disk

TYPE DA

LE

Data

Dir

Item

DBChange

DirChange

Dirop

Root

CONST

DBSize

diskiz

rootDA

rootDA

bott

top

ringsize

di

dir : Dir $:=\{\}$

SDirDA : DA $:=$ bottom

sBusy : DA $\quad:=$ Bottom

busy : DA $\quad:=$ bottom

free : DA := bottom

next : DA $:=$ bottom

buf : Data $:=\{\}$

disk

$\%$ Disk Address in bytes

\% Linear Extent

$\%$ size $=\#$ of bytes

$\%$ Path Name

$\%$ item on the disk

$\% \mathrm{db}$ is data at $\mathrm{x}$ in file $\mathrm{pn}$

$\% \mathrm{x}$ only for Setsize

$\%$ For filling up a $D B$;

$\% \operatorname{Pad}\{x\}$.enc.size $=x$

$\%$ for parsing the busy region

$\%$ assume encoding < DBSize

otDA + DBSize

$=$ (DBSize * diskSize) AS DA

\section{\% smallest DA outside root}

$\%$ starts unlike any Item

$\%$ All volatile; stable data is on disk.

$\%=$ ReadRoot ().dirDA

$\%=$ ReadRoot (). busy

$\%$ DA to write buf at

$\%$ waiting to be written

$\%$ the disk

ABSTRACTION FUNCTION File.dir $=($ LAMBDA $(\mathrm{pn})->$ File.F $=$

$\%$ The file is the data pointed to by the DA's in its F.

$\operatorname{VAR} f:=\operatorname{dir}(\mathrm{pn})$, diskData $:=+:(\mathrm{f} . \mathrm{le}$ * ReadOneDB $)$

RET diskData.seg(0, f.size) )

ABSTRACTION FUNCTION File.oldDirs $=\{\operatorname{SDir}(), \operatorname{dir}\}$

INVARIANT 1: ( ALL $\mathrm{f}$ :IN dir.rng $\mid$ f.le.size * DBSize >=f.size)

$\%$ The blocks of a file have enough space for the data. From FSImpl.

The reason that oldDirs doesn't contain any intermediate states is that the stable state changes only in a sync, which shrinks oldDirs to just dir. 
During normal operation we need to have the variables that keep track of the region boundaries and the stable directory arranged in order around the disk ring, and we need to maintain this condition after a crash. Here are the relevant current and post-crash variables, in order (see below for Minspace). The 'post-crash' column gives the value that the 'current' expression will have after a crash.

\begin{tabular}{lll}
\multicolumn{1}{c}{ Current } & \multicolumn{1}{c}{ Post-crash } \\
busy & sBusy & start of busy region \\
sDirDA & sDirDA & most recent stable dir \\
next & & end of stable busy region \\
free & next & end of busy region \\
free + minSpace () & next + minSpace () & end of cushion for writes
\end{tabular}

In addition, the stable busy region should start and end before or at the start and end of the volatile busy region, and the stable directory should be contained in both. Also, the global variables that are supposed to equal various stable variables (their names start with 's') should in fact do so. The analysis that leads to this invariant is somewhat tricky; I hope it's right.

INVARIANT 2 :

IsOrdered((SEQ DA) \{next + MinSpace(), sBusy, busy, SDirDA, next, free,

Isordered((SEQ Da) (next + Minspace (), busyl)

Finally,

The busy region should contain all the items pointed to from DA's in dir or in global variables.

The directory on disk at SDirDA plus the changes between there and free should agree with dir.

This condition should still hold after a crash.

INVARIANT 3:

$$
\begin{aligned}
& \text { IsAllGood (ParseLog(busy, buf), dir) } \\
& \text { 八 IsAllGood (Parselog(sBusy, \{\}), SDir()) }
\end{aligned}
$$

The following functions are mainly for the invariants, though they are also used in crash recovery. ParseLog expects that the disk from da to the next DB with contents endDB, plus data, is the encoding of a sequence of Item's, and it returns the sequence SI, each Item paired with it DA. ApplyLog takes an SI that starts with a Dir and returns the result of applying all the changes in the sequence to that Dir.

FUNC Parselog (da, data) $\rightarrow$ SI $=$ VAR si, end: DA |

$\%$ Parse the log from da to the next endDB block, and continue with data.

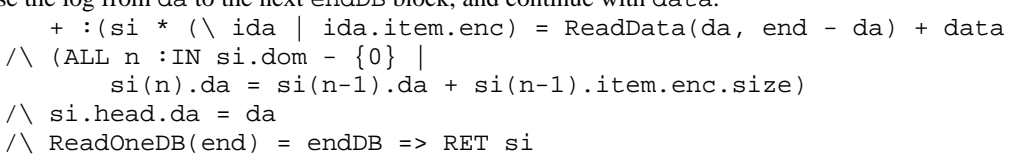


VAR blocks := Disk.DToB(data), $\mathrm{n}:=\mathrm{x} /$ DBSize, $\%$ Extend $f$. le with 0 's to the right length.

le $:=f$ fle + LE.fill(0, $x+$ blocks.size - le.size $)$,

$i:=0$

Do blocks! i $\Rightarrow$

le $(\mathrm{n}+\mathrm{i}):=$ WriteData (DBChange $\{\mathrm{pn}, \mathrm{x}, \operatorname{blocks}(\mathrm{i})\}$.enc) $x+:=$ DBSize; $i+:=1$

OD; $\operatorname{dir}(\mathrm{pn}) .1 \mathrm{e}:=1 \mathrm{e}$

These procedures initialize the system and handle crashes. Crash is somewhat idealized; a more realistic implementation would read the log and apply the changes to dir as it reads them, but the logic would be the same.

PROC Init() = disk := disk.new(diskSize); WriteDir() \% initially dir is empty

PROC Crash ()$=<<$

$\%$ atomic for simplicity

$$
\begin{aligned}
& \text { CRASH; } \\
& \text { SDirDA := ReadRoot().sDirDA; dir }:=\operatorname{SDir}() \\
& \text { sBusy }:=\text { ReadRoot (). busy; busy := sBusy }
\end{aligned}
$$

hese functions read an item, some data, or a single $D B$ from the disk. They are boring. Readitem is somewhat unrealistic, since it just chooses a suitable size for the item at da so that Item. dec works. In real life it would read a few blocks at DA, determine the length of the item from the header, and then go back for more blocks if necessary. It reads either from buf or from the disk, depending on whether da is in the write buffer, that is, between next and free.

FUNC ReadItem(da) $\rightarrow$ Item $=$ VAR size: $\mathrm{x}$

RET Item.dec( ( DABetween (da, next, free) $\Rightarrow$ buf.seg (da - next, size)

[*] ReadData (da, size) ) )

FUNC ReadData (da, size: $\mathrm{X}) \rightarrow$ Data $=$

IF size + da $<=$ top $\Rightarrow$

$\%$ Read the necessary disk blocks, then pick out the bytes requested.

$\% 1$ or 2 disk.read's

$$
\text { RET data.seg(da//DBSize, size) }
$$

ReI data.seg(da//DBsize, size)

[*] RET ReadData (da, top - da) + ReadData (bottom, size - (top - da))

$\mathrm{PROC}$ ReadOneDB(da) $=\operatorname{RET}$ disk.read $(\operatorname{LE}\{d a / D B S i z e, 1\}))$

WriteData writes some data to the disk. It is not boring, since it includes the write buffering, the cleaning, and the space bookkeeping. The writes are buffered in buf, and sync does the actual disk write. In this module Sync is only called by WriteDir, but since it's a procedure in File it can also be called by the client. When writeData needs space it calls clean, which does the basic cleaning step of copying a single item. There should be a check for a full disk, but we omit it. This check can be done by observing that the loop in WriteData advances free all the way around the ring, or by keeping track of the available free space. The latter is fairly easy, but Crash would have to restore the information as part of its replay of the log.

These write procedures are the only ones that actually write into buf. Sync and WriteRoot below are the only procedures that write the underlying disk.
PROC WriteData(data) $\rightarrow$ DA $=$

DO IsFull (data.size) $\Rightarrow$ Clean() OD;

buf $+:=$ data; VAR da $:=$ free $\mid$ free $+:=$ data.size; RET da

PROC WriteItem(item) = VAR d $:=$ item.enc $\mid$ buf $+:=$ di free $+:=$ d.size $\%$ No check for space because this is only called by Clean, WriteDir.

PROC Sync() =

$\%$ Actually write to disk, in 1 or 2 disk.write's (2 if wrapping).

$\%$ If we will write past sBusy, we have to update the root.

IF (sBusy - next) + (free - next) $<=$ MinSpace ()$\Rightarrow$ WriteRoot () [*] SKIP FI; \% Pad buf to even DB's. A loop because one Pad might overflow current DB.

DO VAR z := buf.size//DBSize $\mid$ z \# 0 => buf := buf + Pad $\{$ DBSize-z $\}$.enc OD;

$$
\% \text { add the end marker DB }
$$$$
\text { buf := buf + endDB; }
$$

$<\%$ atomic for simplicity

IF buf.size + next $<$ top $\Rightarrow$ disk.write (next/DBSize, buf)

[*] disk.write(next /DBSize, buf.seg(0, top-next));

disk.write (bottom/DBSize, buf.sub(top-next, buf.size-1))

FI

>> free $:=$ next + buf.size - DBSize; next $:=$ free; buf $:=\{\}$

The constraints on using free space are that clean must not cause writes beyond the stable sBusy or into a disk block containing Item's that haven't yet been copied. (If sBusy is equal to busy and in the middle of a disk block, the second condition might be stronger. It's necessary because a write will clobber the whole block.) Furthermore, there must be room to write an Item containing dir. Invariant 2 expresses all this precisely. In real life, of course, clean would be called in the background, the system would try to maintain a fairly large amount of free space, and only small parts of dir would be dirty. Clean drops DirChange's because they are recorded in the Dir item that must appear later in the busy region.

FUNC IsFull(size: X) $\rightarrow$ Bool = RET busy - free < MinSpace () + size

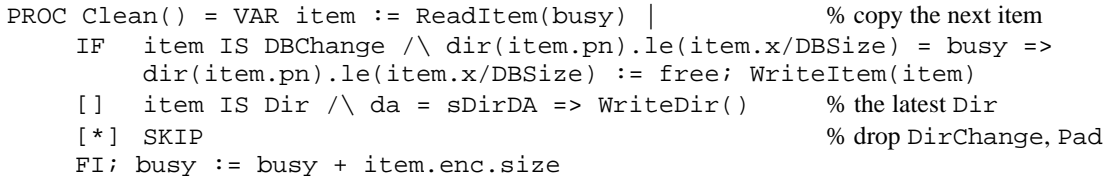

$\%$ Called only from Clean and Init. Could call it more often to speed up recovery

$\%$, after DO busy - free < MinSpace () => Clean() OD to get space.

sDirDA := free; WriteItem(dir); Sync(); WriteRoot()

The remaining utility functions read and write the root, convert byte sizes to DB counts, and provide arithmetic on DA's that wraps around from the top to the bottom of the disk. In real life we don't need the arithmetic because the disk is divided into segments and items don't cross segment boundaries; if they did the cleaner would have to do something quite special for a segment that starts with the tail of an item. 
FUNC ReadRoot ()$\rightarrow$ Root $=$ VAR root, pad $\mid$ ReadOneDB (rootDA) = root.enc + pad.enc $=>$ RET root

PROC WriteRoot ()$=<$ VAR pad, $d b \mid d b=\operatorname{Root}\{s D i r D A$, busy $\}$.enc + pad.enc $=>$ disk.write(rootDA, db); sBusy := busy >>

FUNC NumDBs(da, size: X) $\rightarrow$ Int $=$ RET $($ size + da//DBSize + DBSize-1)/DBSize $\%$ The number of DB's needed to hold size bytes starting at da.

FUNC DAAdd (da, $i$ : Int $) \rightarrow D A=\operatorname{RET}((d a-$ bottom $+i) / /$ ringSize $)+$ bottom

FUNC DASub (da, i: Int) $\rightarrow$ DA $=\operatorname{RET}(($ da - bottom - i) // ringSize) + bottom $\%$ Arithmetic modulo the data region. abs (i) should be $<$ ringsize.

FUNC DABetween $($ da, da1, da2) $->$ Bool $=$ RET da $=$ da1 $\backslash /($ da2 - da1 $)<($ da1 - da $)$

FUNC IsOrdered(s: SEQ DA) $\rightarrow$ Bool $=$

RET (ALL i : IN s.dom - $\{0,1\} \mid$ DABetween(s(i-1), s(i-2), s(i))

END CopyingFS 